\begin{abstract}
HOGGAN, Erik Nebeker. Spin coating and photolithography using liquid and supercritical carbon dioxide. (Under the direction of Dr. Ruben G. Carbonell and Dr. Joseph M. DeSimone.)

This thesis details work on the utilization of dense phase carbon dioxide $\left(\mathrm{CO}_{2}\right)$ in semiconductor processing. In particular, work is presented on the formulation of $\mathrm{CO}_{2}$ soluble photoresists and the spin coating of those photoresists using only liquid $\mathrm{CO}_{2}$ as a solvent. As part of this spin coating work, a novel high-pressure $\mathrm{CO}_{2}$ spin coater was designed and constructed, and the theoretical equations governing its performance were derived. Also discussed in this thesis are 248 and $193 \mathrm{~nm}$ exposures of these $\mathrm{CO}_{2}$ spun films and subsequent development in supercritical $\mathrm{CO}_{2}$. Resist stripping was also performed in $\mathrm{CO}_{2}$. In short, this thesis details the first steps towards a complete replacement of all aqueous and organic solvents in the conventional photolithographic processes of spin coating, developing, and resist stripping. This change not only confers significant environmental advantages, but opens up many new avenues in resist chemistry and promises improvements in large scale film uniformity, elimination of feature collapse, elimination of extraneous processing steps, and improved control of the lithographic process.
\end{abstract}




\title{
SPIN COATING AND PHOTOLITHOGRAPHY USING LIQUID AND SUPERCRITICAL CARBON DIOXIDE
}

\author{
by

\section{ERIK NEBEKER HOGGAN} \\ A thesis submitted to the faculty of \\ North Carolina State University \\ in partial fulfillment of the \\ requirements for the Degree of \\ Doctor of Philosophy \\ CHEMICAL ENGINEERING
}

Raleigh

2002

APPROVED BY:

Ruben Carbonell (co-chair)

Joseph DeSimone (co-chair)

Peter Kilpatrick

Gregory Parsons

Christine Grant 


\section{BIOGRAPHY}

Erik Nebeker Hoggan was born March 18, 1974 in San Jose, CA. He moved to Colorado Springs, CO, at an early age and completed his high school years there. In high school he was active in the athletic and music departments, playing varsity soccer and being named first chair tuba at the Colorado all-state band. Erik graduated from Cheyenne Mountain High School as a National Merit scholar and attended Brigham Young University in Provo, UT, for a year. In 1993 he married Julie Lyn Brown and transferred to the University of Colorado at Boulder, where he obtained his B.S. in Chemical Engineering in 1996. While at CU, Erik was named Undergraduate Researcher of the Year in 1995 for his assistance with work on polymer matrices in ferroelectric liquid crystals.

In August 1996 Erik entered the graduate program at North Carolina State University. He received his Masters in Chemical Engineering in 1998 under Ruben G. Carbonell and Joseph M. DeSimone. His work at NCSU began in the area of metal cleaning in $\mathrm{CO}_{2}$ and progressed to coatings and photolithography in $\mathrm{CO}_{2}$. Erik and his wife Julie have three children, Emma, Madeline, and Samuel. Erik will begin his professional career at the Intel Corporation (Hillsboro, OR) in the area of photolithography research. 


\section{ACKNOWLEDGEMENT}

I would first thank my two advisors, Ruben Carbonell and Joe DeSimone, for their invaluable advice and aid throughout my graduate work. Even as I near the end of my graduate career, I am still amazed at their depth of knowledge and insights on so many different topics. I thank Devin Flowers and Jonathan Kendall for their work on the chemistry related to this project, without whom this work would not have been possible.

I thank Joe Royer and Flory Henon, who taught me how graduate research was done, and made the lab a fun place to come to every day. I thank everyone at the $\mathrm{CO}_{2}$ Center for their friendship and assistance, especially Brian Novick for the many helpful research discussions. I thank Chris Bowman and Alan Guymon, both formerly of the University of Colorado, without whom I never would have sought out a Ph.D in the first place.

I thank my parents, Jim and Rosalind Hoggan for their unending support and encouragement. And finally, I thank my wife, Julie, without whom I would not have

finished this work, and who suffered cheerfully with me through the poverty of graduate school these many years. 


\section{TABLE OF CONTENTS}

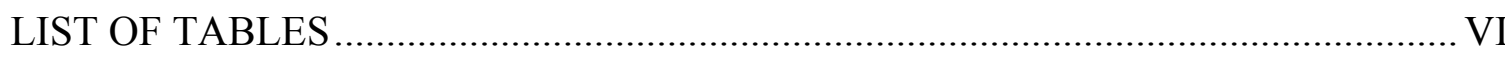

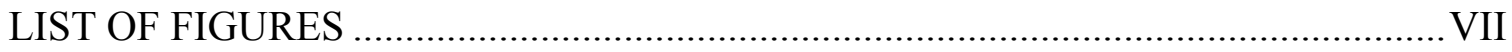

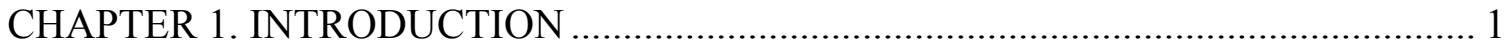

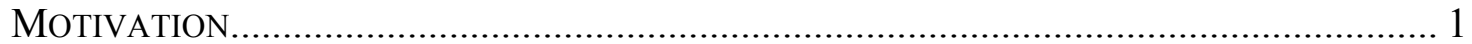

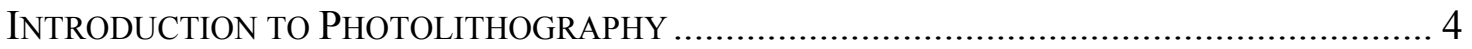

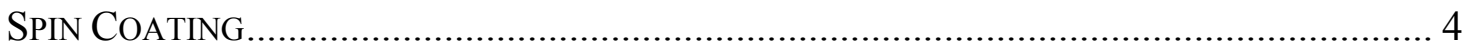

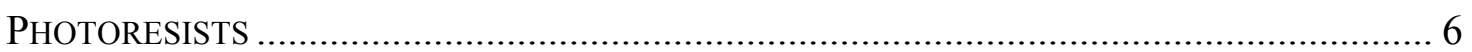

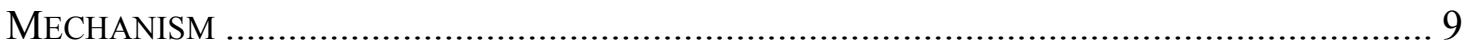

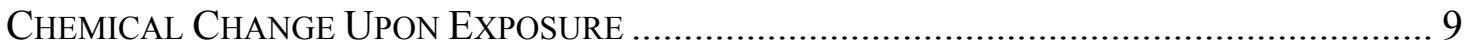

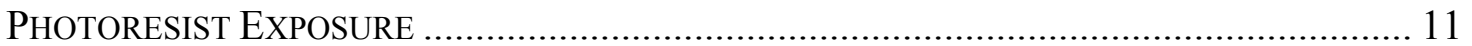

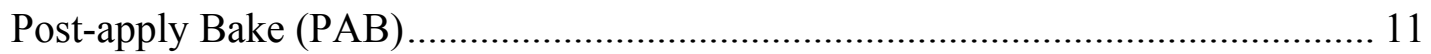

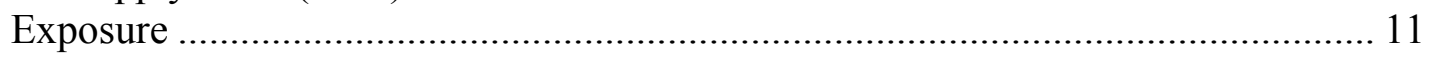

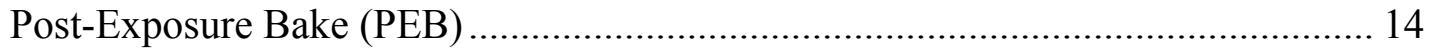

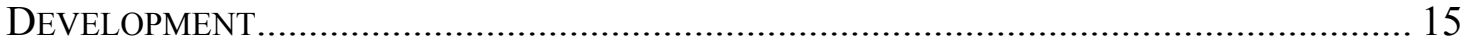

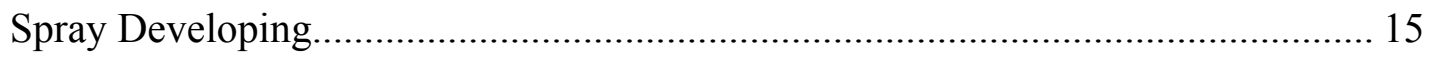

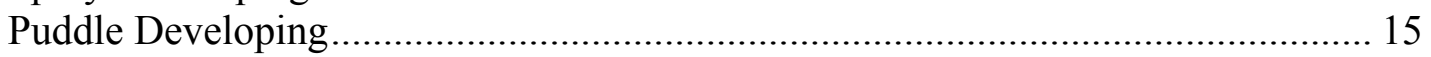

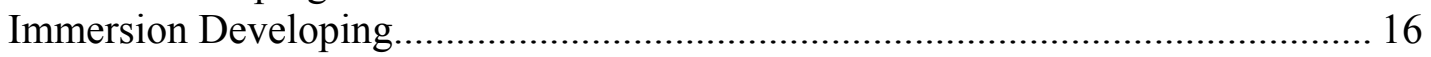

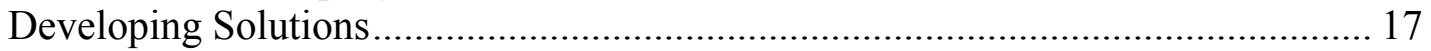

Photoresist Characterization …………………………..................................... 17

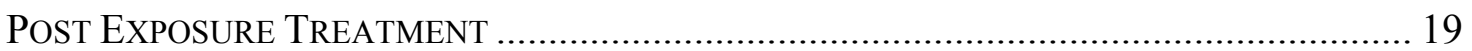

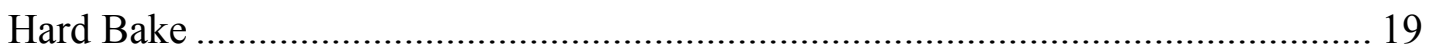

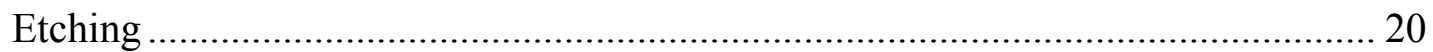

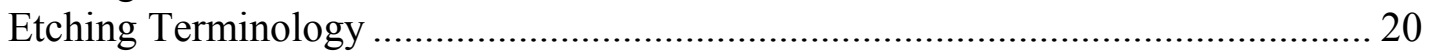

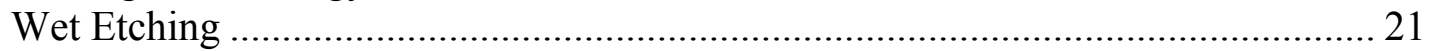

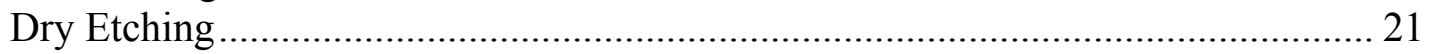

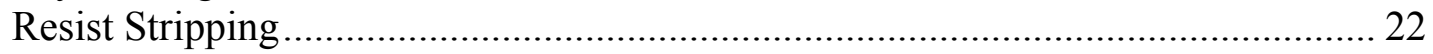

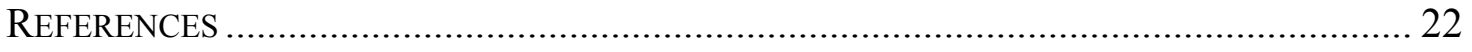

CHAPTER 2. APPLICABILITY OF $\mathrm{CO}_{2}$ TO PHOTOLITHOGRAPHY ..................... 33

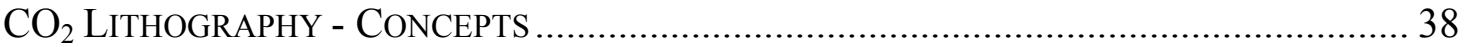

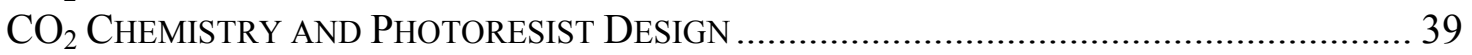

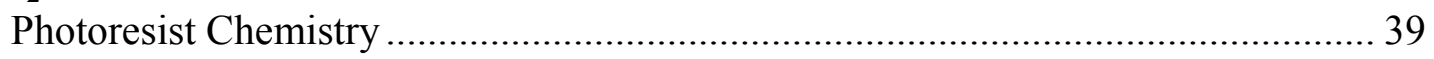

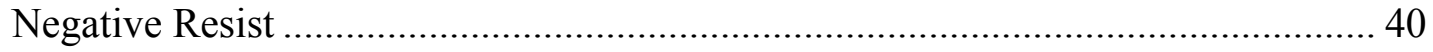

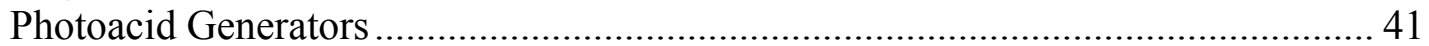

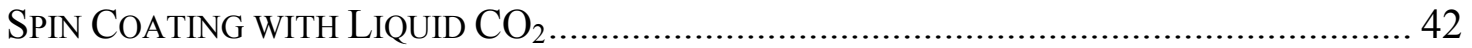

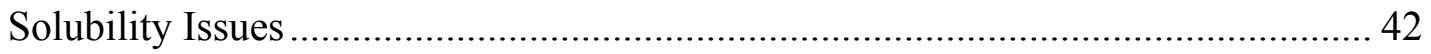

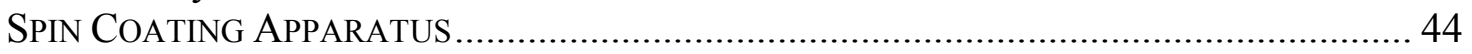

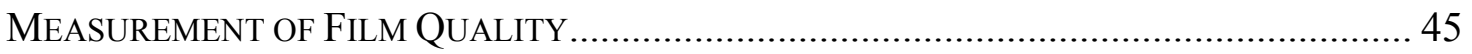

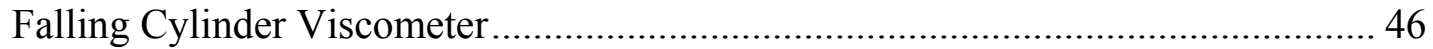

Solubility Measurements .................................................................................... 49 


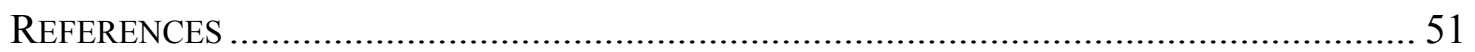

CHAPTER 3. $\mathrm{CO}_{2}$ SPIN COATING APPARATUS, THEORY, AND COATING

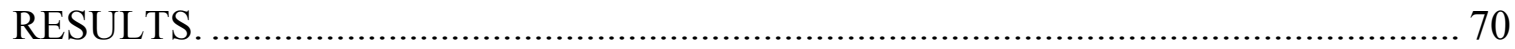

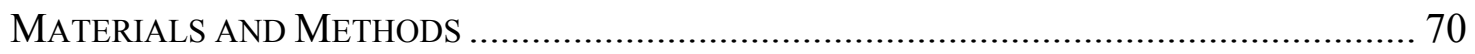

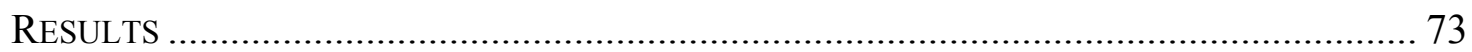

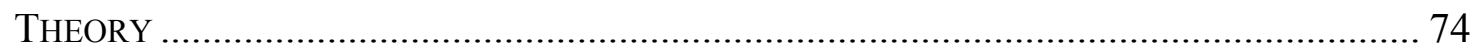

COMPARISON OF THEORY AND EXPERIMENT ………….......................................... 79

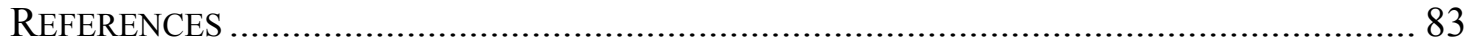

CHAPTER 4. EXPOSURE AND DEVELOPMENT OF $\mathrm{CO}_{2}$ BASED PHOTORESIST

92

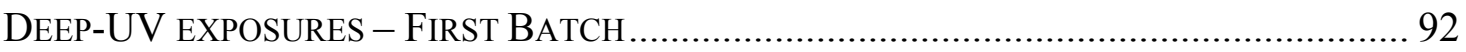

DEEP-UV EXPOSURES - IMPROVED RESULTS ........................................................ 98

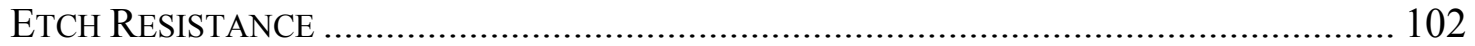

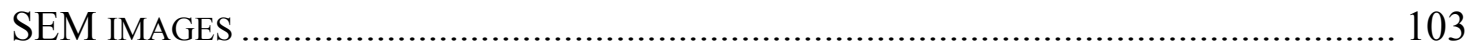

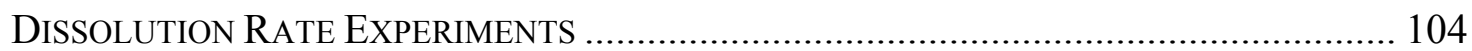

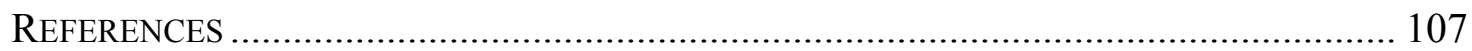

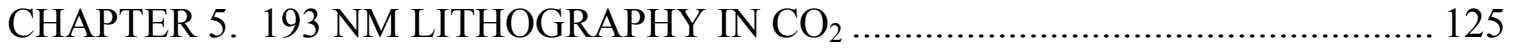

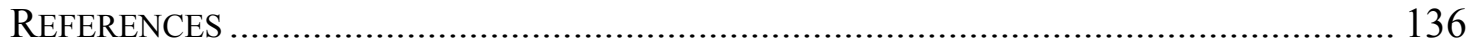

CHAPTER 6. CONCLUSIONS AND FUTURE DIRECTIONS ………….................. 145

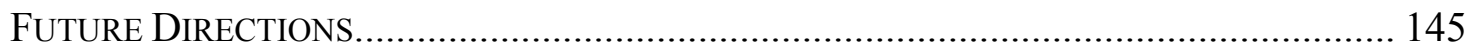

APPENDIX A. ANALYSIS OF SPIN COATING IN LIQUID CO $\mathrm{CO}_{2} \ldots \ldots \ldots \ldots \ldots \ldots \ldots \ldots \ldots \ldots . . . . . . . . . . .147$

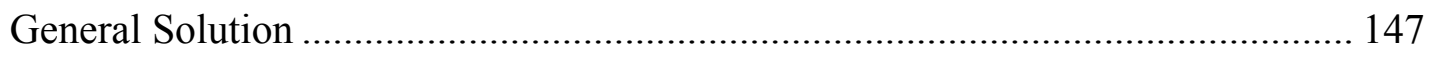

Special Solution for the Case of an Initially Uniform Distribution ......................... 150

Significance of Analysis ................................................................................ 150

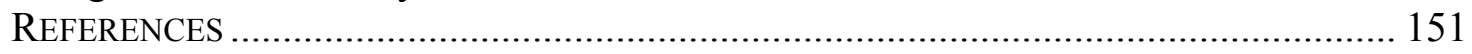

APPENDIX B. DESIGN OF THE 8" $\mathrm{CO}_{2}$ SPIN COATER ……………................... 152

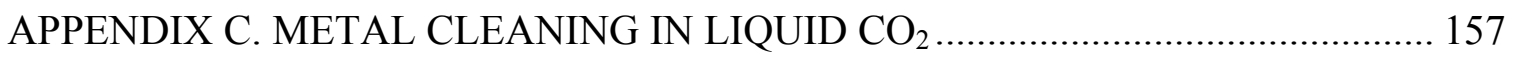

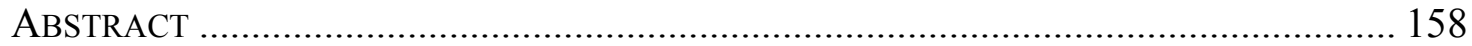

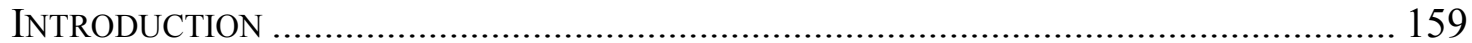

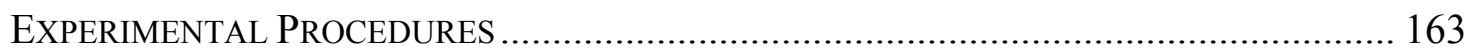

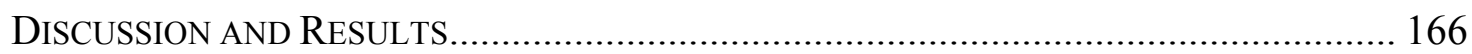

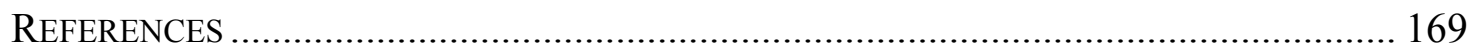




\section{LIST OF TABLES}

Table 1.1. Classes of photoresist reactions. .............................................................. 9

Table 2.2. Properties of Poly(FOMA-r-THPMA) resins used..................................... 41

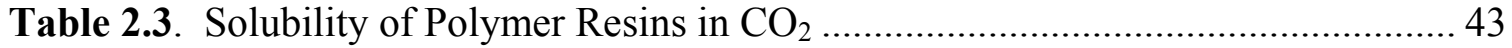

Table 3.2. Solubilities of poly(FOMA-r-TBM)resins used......................................... 71

Table 3.3. Thickness of Poly(FOMA-r-THPMA) films at various conditions.............. 73

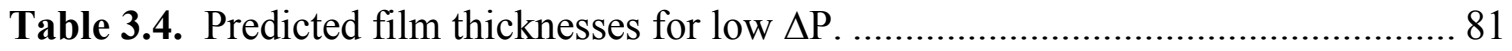

Table 4.1. Swelling of various copolymer resins over the course of 14 hours.............. 95

Table 4.2. Copolymer resins used in initial flood exposure work................................ 95

Table 4.3. Film thicknesses and PAG loadings.......................................................... 96

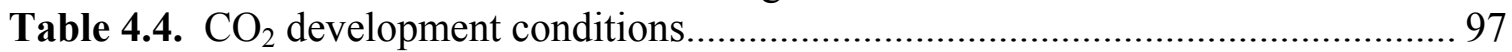

Table 4.5. Experimental conditions used in the second round of deep-UV exposures. 100

Table 4.6. Etching rates of several photoresist formulations. ..................................... 103

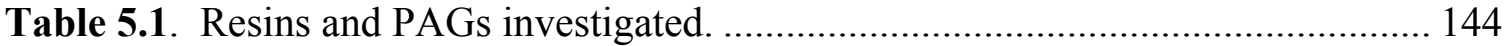

Table C.1. Physical Data for Liquid Carbon Dioxide and Other Solvents ................... 160 


\section{LIST OF FIGURES}

Figure 1.1. Positive and negative resist exposure and development.............................. 27

Figure 1.2. Schematic of a reduction step-and-repeat projection system ....................... 28

Figure 1.3. Sensitivity plot of positive and negative photoresists. ................................. 29

Figure 1.4. Lateral etch dimensions............................................................................ 30

Figure 1.5. Illustration of overetching on undercutting and sidewall slope.................... 31

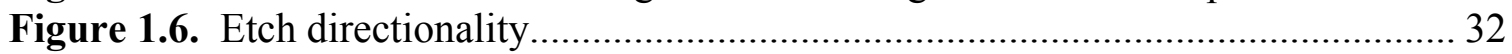

Figure 2.1. Density, surface tension and viscosity of liquid $\mathrm{CO}_{2}$ vs temperature. ......... 56

Figure 2.2. Density of dense phase $\mathrm{CO}_{2}$ vs pressure . .............................................. 57

Figure 2.3. Schematic of how $\mathrm{CO}_{2}$ may be used for spinning, developing and stripping

by altering the solvent strength for each step....................................................... 58

Figure 2.4. Structure of negative resist copolymer ......................................................59

Figure 2.5. Structure of $\mathrm{CO}_{2}$ soluble photoresist system ............................................... 60

Figure 2.6. Liquid $\mathrm{CO}_{2}$ density vs pressure at various temperatures...............................61

Figure 2.7a. Overview of liquid $\mathrm{CO}_{2}$ spin coating apparatus........................................6. 62

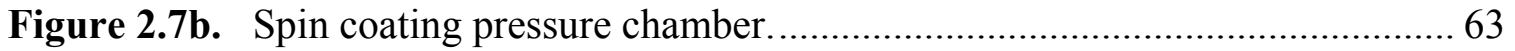

Figure 2.7c. Key components of the spin coating apparatus. ....................................... 64

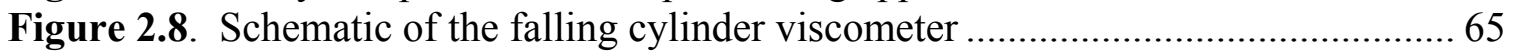

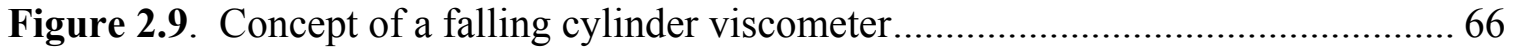

Figure 2.10. Calibration plot for the falling cylinder viscometer. .................................. 67

Figure 2.11. Viscosity of $\mathrm{CO}_{2} /$ poly(FOMA-r-TBM)solutions measured with the falling

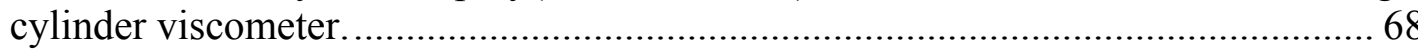

Figure 2.12. Plot of Reynolds number vs. $\phi$, demonstrating the stability limit of spun films.

Figure 3.1. Synthesis of poly(FOMA-r-TBM)in supercritical $\mathrm{CO}_{2} \ldots \ldots \ldots \ldots \ldots \ldots \ldots \ldots \ldots \ldots \ldots . . . . . . \ldots 5$

Figure 3.2. Viscosity of $\mathrm{CO}_{2} /$ poly(FOMA-r-TBM)solutions measured with the falling

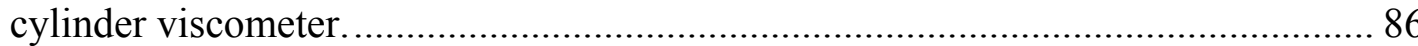

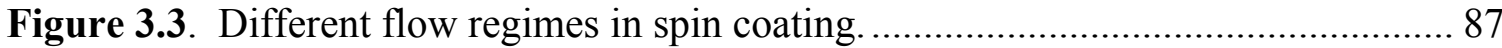

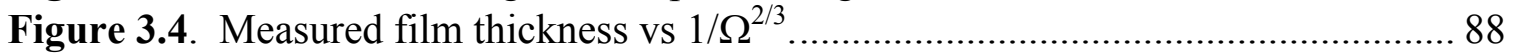

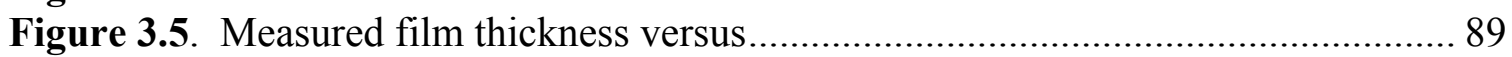

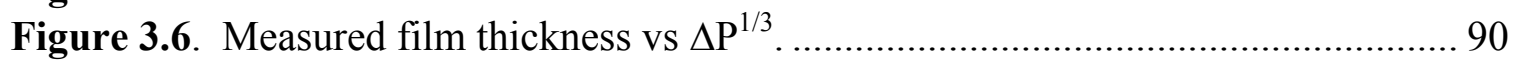

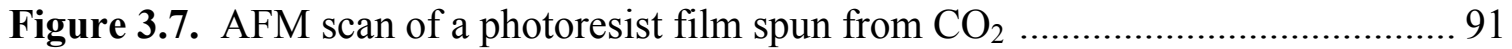

Figure 4.1. Structure of $\mathrm{CO}_{2}$ soluble photoresist system ......................................... 108

Figure 4.2. Sample frame measuring the swelling of TBM copolymer resins in $\mathrm{CO}_{2}$ at

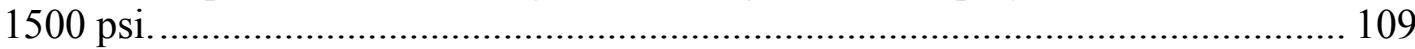

Figure 4.3. Swelling of three polymer resins in $\mathrm{CO}_{2}$ at $1500 \mathrm{psi}$............................. 110

Figure 4.4. Dose matrix (with values in $\mathrm{mJ} / \mathrm{cm}^{2}$ ) used for samples ........................... 111

Figure 4.5. Schematic of the $\mathrm{CO}_{2}$ development chamber ........................................ 112

Figure 4.6. Sensitivity curve for sample 6, CLM-A-015 w/ 5\% ionic PAG and sample 7, CLM-A-003 w/ 3\% ionic PAG.

Figure 4.7. Sensitivity curve for sample 7, CLM-A-009 w/ 4\% ionic PAG, and sample 8, CLM-A-009 w/ 4\% ionic PAG. 
Figure 4.8. Sensitivity plots for three different MW resins at 5\% ionic PAG loading, PEB of $90^{\circ} \mathrm{C}$

Figure 4.9. Sensitivity plots for three different PEB temperatures. All films were of resin $\mathrm{C}$ at $5 \%$ ionic PAG loading.

Figure 4.10. Sensitivity plot of resin B, with 5\% ionic PAG and 5\% nonionic PAG... 117

Figure 4.11. UV-Vis absorbance of the two photoacid generators............................. 118

Figure 4.12. SEM images of $248 \mathrm{~nm}$ exposed features............................................ 120

Figure 4.14. Schematic of the rotating disk dissolution monitoring apparatus............. 121

Figure 4.15. Relevant effects in the dissolution rate monitoring experiments............ 122

Figure 4.16. a) Remaining film thickness as a function of rotational speed; b) removed film thickness as a function of operating pressure

Figure 4.17. Portion of film removed plotted versus the square root of rotational speed..

Figure 5.1. Structure of $\mathrm{CO}_{2}$ soluble photoresist system

Figure 5.2. Schematic of how $\mathrm{CO}_{2}$ may be used for spinning, developing and stripping by altering the solvent strength for each step.

Figure 5.3. a) Sensitivity plot for $15 \mathrm{k}$ MW PFOMA- $r$-THPMA with both ionic and nonionic PAGs, b) Sensitivity plot for 25k MW PFOMA- $r$-THPMA with both ionic and nonionic PAGs.

Figure 5.4. $248 \mathrm{~nm}$ exposures of $25 \mathrm{k}$ MW Poly(FOMA-r-THPMA) photoresist with $5 \%$ ionic PAG.

Figure B.1. Opening mechanism for the 8" spin coating vessel................................ 154

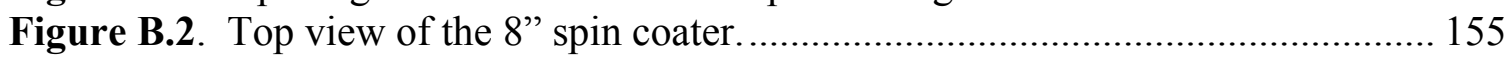

Figure B.3. Dimensions of the 8" spin coater - side view........................................ 156

Figure C.1. Phase Diagram for Carbon Dioxide ………………………………....... 170

Figure C.2. Weight percent of oil removed as a function of time in aqueous system... 171

Figure C.3. Weight percent of oil removed as a function of time in carbon dioxide. ... 172

Figure C.4. Weight percent of oil removed as a function of time in three solvents..... 173

Figure C.5. Weight percent of oil removed as a function of time in carbon dioxide. ... 174 


\section{Chapter 1. Introduction}

\section{Motivation}

Throughout the last several decades, photolithography has evolved to keep pace with the rapid changes in the semiconductor industry. The technology has evolved from Novolac based resists to chemically amplified deep-UV resists to $193 \mathrm{~nm}$ systems. However, despite the drastic changes in chemistry, optics, and processing techniques, there has been relatively few improvements in the solvents used in these processes. The majority of lithography continues to use organic solvents for spin coating and resist stripping, and aqueous basic solutions such as tetramethyl-ammonium hydroxide (TMAH) for image development. These solvents and conventional methods have served the industry well for years, however, as feature sizes continue to shrink and exposure wavelengths drop to 157 $\mathrm{nm}$ and below, there are compelling reasons to reevaluate these processes and implement a new technology.

As the semiconductor industry progresses to the $157 \mathrm{~nm}$ wavelengths and beyond, problems have arisen with regards to image collapse, optical density of photoresists, and the uniformity of coatings on large 12 inch wafers. Carbon dioxide is not only poised to alleviate all these problems, but also has the significant advantages of being environmentally benign, relatively inexpensive, and recyclable. The work presented here deals with utilizing this novel solvent to eliminate many of the problems inherent in conventional solvents and opening the door for many other improvements in photolithography. 
This research focuses on the most solvent intensive steps of semiconductor processing; spin coating of photoresists, development of the imaged wafers, and resist stripping. The first of these steps, spin coating, typically uses hazardous organic solvents such as 2-ethoxy ethyl acetate and diglyme. While the amount of solvent used has decreased in recent years due to tighter controls and improved equipment, a typical fab line still consumes about 1 million gallons of organic solvents a year at a cost of $\$ 400 \mathrm{M}$ [1]. This figure does not include the costs associated with ventilation equipment, hazardous waste disposal, and safety measures that must be used when handling these solvents that are hazardous to both workers and the environment.

In addition to spin coating solvents, wafer processing utilizes large amounts of aqueous solutions of bases such as tetramethylammonium hydroxide (TMAH) for photoresist developing. For example, a large semiconductor processing line which produces 5,000 wafers per day will generate almost 2000 gallons per day of waste developing solution and nearly an equivalent amount of contaminated rinse water [2]. After etching, wafers undergo a final solvent intensive process wherein the remaining photoresist is stripped from the surface using solvents similar to those used in spin coating.

This research was initially motivated by the opportunity to replace these hazardous and expensive solvents with a more environmentally friendly alternative, compressed carbon dioxide $\left(\mathrm{CO}_{2}\right)$. Recognizing the potential of $\mathrm{CO}_{2}$ and the high solubility of fluoroacrylate polymers in $\mathrm{CO}_{2}$ [3], several groups have recently used supercritical $\mathrm{CO}_{2}\left(\mathrm{scCO}_{2}\right)$ in the development of photoresist films . Allen, Ober and 
others have shown that in some cases it is possible to replace the traditional aqueous developers with $\mathrm{scCO}_{2}[4],[5],[6],[7]$. However, to date, no one has addressed the issue of replacing the hazardous organic solvents used in the spin coating process, nor has anyone designed a $\mathrm{CO}_{2}$ soluble photoresist that could be stripped using supercritical $\mathrm{CO}_{2}$.

This thesis deals with the replacement of all aqueous and organic solvents throughout the photolithographic process. Work has been done on the design and construction of first and second generation $\mathrm{CO}_{2}$ spin coating devices, empirical and theoretical investigations of the $\mathrm{CO}_{2}$ spin coating process, image exposure, image development and finally photoresist stripping.

This dissertation begins with an introduction to the concepts and theory associated with conventional photolithography, and the distinct advantages of using liquid and supercritical $\mathrm{CO}_{2}$. The following chapters present the work done in spin coating, modeling, development, equipment design, and generation of actual lithographic images using only $\mathrm{CO}_{2}$ as the solvent. 


\section{Introduction to Photolithography}

Prior to discussing the details of implementing photolithography in $\mathrm{CO}_{2}$, it is necessary to review the methods and techniques used in the current manufacturing process. Of primary concern are the solvent, both organic and aqueous, intensive steps, spin coating, pattern development, and stripping. Inclusive in the discussion are various processing steps that are necessary to semiconductor processing and must also be considered when developing alternate chemistries and procedures.

\section{Spin Coating}

Spin coating is a process in which a thin liquid film is spread by centrifugal force onto a rotating substrate. Spin coating is used to form thin $(0.3-10 \mu \mathrm{m})$ films with nonuniformities no greater than $1 \%[8]$. When the spin coated liquid contains polymers or colloids (up to $25 \mathrm{wt} \%$ solute), solid films may be formed on the substrate [9],[10]. Spin coating was first used extensively in the 1950s to deposit phosphor onto the glass surfaces of color television tubes [11]. Currently, the most common application for such films is in the microelectronic industry, but the technique is also used in the manufacture of compact discs, flat panel displays, and highly reflecting mirrors [12].

The first detailed mathematical analysis of spin coating was conducted by Emslie, Bonner, and Peck [13]. In their analysis, they neglected the effect of evaporation and the resultant viscosity and concentration gradients. Meyerhofer expanded upon their analysis 
and those results have been extended even more recently by Bornside et al. and Yonkoski et al. [14],[15],[16].

The conclusion reached by many of the above authors is that the spin coating process can be generally considered to proceed in four phases. In the first stage the liquid solution is deposited on the substrate, covering the entire surface. In the second stage, spin-up, the rotational speed of the substrate is rapidly increased to its final value. Acceleration rates of $10,000 \mathrm{rpm} / \mathrm{s}$ are common in the spin-up stage. Ideally, very little film thinning occurs in this step. The two final steps, spin-off and evaporation, occur concurrently. Final rotation speeds of 5,000 to $10,000 \mathrm{rpm}$ are common. At the beginning of the spin-off step, the film is several microns thick and the fluid lost to centrifugal force is far greater than that lost to evaporation. However, as the film becomes thinner, relatively little solution is flung off the wafer and evaporation becomes the primary method of film thinning. The spin coating process is typically completed in less than a minute [17],[18].

The analyses conducted by these authors have been shown to hold for a variety of volatile organic solvents. One of the earliest proposed models for this process is explained in detail in Appendix A [13]. This model relates the theoretical film thickness to physical parameters such as the solution viscosity, density, spinning time, and rotational speed. The end result of the analysis is an expression for the final wet film height as a function of the process variables, 


$$
H=\frac{H_{i}}{\left(1+4 \frac{\rho \Omega^{2}}{3 \eta} H_{i}^{2} t\right)^{\frac{1}{2}}} .
$$

Here, $H_{0}$ is the initial film height, $t$ is the spinning time, $\rho$ is the solution density, $\eta$ is the solution viscosity, and $\Omega$ is the rotational speed. Even though equation 1.1 neglects the effects of solvent evaporation, it does indicate that higher rotational speeds and lower viscosities yield thinner films at any given time. The agreement between this theory, a modified theory applicable to $\mathrm{CO}_{2}$ spin coating, and measured film thicknesses is the major focus of Chapter 3.

\section{Photoresists}

Photolithography is the process in which images or patterns are created in thin polymer films on semiconductor wafers. The first step in this process is the spin coating of thin films of photosensitive polymers, known as photoresists. These photoresists undergo chemical changes upon exposure to specific wavelengths of light.

Photoresist materials have their origins in the history of photography, which began with Niepce in the early $19^{\text {th }}$ century [19]. In his experiments with bitumen of Judea, he pioneered the idea of making an image by taking advantage of light-induced solubility changes. Niepce later used more light sensitive materials such as dichromated gelatin.

A century later, when William Shockley and coworkers at Bell Laboratories began work on the first integrated circuits, they also began with dichromated gelatin. As a photoresist, it possessed adequate resolution, but it was an ineffective resist for 
hydrofluoric acid etching. As a result of their work, Louis Minsk initiated the use of poly(vinylcinnamate), which was used for the production of the first semiconductor devices. However, due to lack of adhesion of the polymer to silicon, device yield was poor. Kodak responded by creating a new resist based on bis-azides and rubber, which had excellent adhesion properties. This resist remained the industry standard until the early ' 70 s[19].

To meet the demand for decreasing feature sizes, the semiconductor industry switched to a diazonaphthoquinone (DNQ) resists created by Oskar Sü $\beta[19]$. DNQ is used in a mixture with cresol-formaldehyde condensation polymers (novolacs). These alkali-soluble novolac binders are rendered insoluble when mixed with DNQ. However, when exposed to light, DNQ is converted to indene-3-carboxylic acid (sufficient water for the reaction is usually present in the thin film)[20].

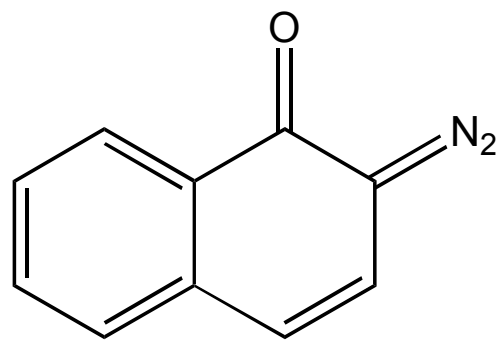

diazonaphthoquinone

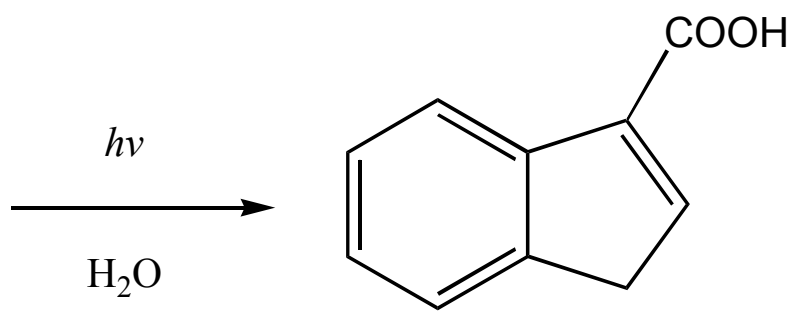

indene-3-carboxylic acid

The indene carboxylic acid is soluble in aqueous alkali solution, causing the exposed portions of the photoresist to dissolve and forming a positive image on the wafer. Thus the solubility of the material is controlled by selective irradiation. The DNQ-novolac 
system has remained the industry workhorse for nearly two decades, and it is still the resist system of choice in many applications.

The example cited above is one of the many photoresist systems used. All photoresists undergo a chemical change upon exposure to light, but the nature of the change is different from one resist to another. In general, polymeric photoresists experience one of three possible changes upon exposure to a radiation source. The possible reactions are enumerated in Table 1.1. 
Table 1.1. Classes of photoresist reactions.

\begin{tabular}{|c|c|}
\hline Mechanism & Chemical Change Upon Exposure \\
\hline Chain Scission & $\begin{array}{l}\text { High molecular weight (MW) polymer chains are broken } \\
\text { down into lower MW chains. }\end{array}$ \\
\hline Cross-linking & $\begin{array}{l}\text { Monomer or low MW polymer reacts with itself to form a } \\
\text { cross-linked network. A sensitizer is often used to } \\
\text { initiate the reaction. }\end{array}$ \\
\hline Chemical Modification & $\begin{array}{l}\text { a) The polarity of the polymer or an end group is } \\
\text { changed. For example, a polar end group may be } \\
\text { formed. } \\
\text { b) In photoresist systems that contain an inhibitor, } \\
\text { exposure induces a chemical transformation which } \\
\text { renders the inhibitor inactive, allowing the polymer } \\
\text { to dissolve. } \\
\text { c) The chemical structure of the polymer is changed. } \\
\text { For example, oxidation or chemical rearrangement } \\
\text { alters the nature of the polymer. }\end{array}$ \\
\hline
\end{tabular}

As can be seen from Table 1.1, there are a large variety of potential reactions, however, it is possible to classify all photoresists as one of two classes; negative and positive resists. Positive resists are photoresists that become more soluble in the developing solution upon exposure. These resists may undergo chain scission, have inhibitors that are rendered inactive when exposed, or may undergo a variety of end group transformations to alter their solubility. The use of such resists results in a wafer image that is identical to that on the mask. Photoresists that become less soluble in the 
developing solution upon exposure are termed negative resists because the resultant wafer image is opposite that of the mask. These resists may cross-link or experience various structural changes to render them less soluble. The two different resist types are shown in Figure 1.1. The DNQ-novolac system described earlier is a positive resist.

As DNQ-novolac systems reached their performance limit and the industry switched to deep UV exposure wavelengths, some refinements to the photolithographic process became necessary. Early deep UV light sources were much weaker than the older g-line and i-line sources, so deep UV resists had to be far more sensitive to light than older DNQ resists. This problem was solved by the introduction of chemically amplified resists which used acid catalysis [21], [22]. Chemically amplified resists are two component resists containing a polymer resin and a photoacid generator (PAG). The polymer resin contains protecting groups that alter the solubility of the polymer, but are easily removed in the presence of an acid. The PAG supplies this acid when it is exposed to radiation of the proper wavelength. The cleavage of the protecting groups often requires elevated temperatures in addition to the presence of the acid, but it is catalytic. Thus, after cleavage of one protecting group, additional acid is generated and is available to cleave more leaving groups. The catalytic nature of the reaction allows a single PAG molecule to cleave dozens or even hundreds of protecting groups, resulting in a much more light sensitive resist than DNQ systems. As exposure wavelengths have decreased from deep UV to $193 \mathrm{~nm}$ to $157 \mathrm{~nm}$, chemical amplification has remained the dominant mechanism for high performance resists. The remainder of the photoresists described in this thesis operate on this same principle. 


\section{Photoresist Exposure}

Several processing steps occur between the spin coating of a film and its development. These processes are solvent-free and do not use $\mathrm{CO}_{2}$ directly, but they illustrate several of the desired properties of polymer photoresists. Thus, must be considered when developing a novel resist system.

Post-apply Bake(PAB)

After conventional spin coating, wafers undergo a post-apply bake (PAB), or softbake, to remove any residual organic solvent and to increase adhesion of the resist to the substrate [23]. Another important function of this baking step is to anneal the film and decrease free volume in the polymer. This annealing not only produces a denser, more uniform film, but also serves to minimize environmental contamination of the film by reducing the absorption of bases from the atmosphere. This soft-bake is typically carried out at $80{ }^{\circ} \mathrm{C}-120^{\circ} \mathrm{C}$ for about 1 minute. Because $\mathrm{CO}_{2}$ is a gas at ambient pressures, it was not necessary to heat the films spun in this work to drive off residual solvent. For that reason, a PAB was not used in this work. Later work may show that this step should be reintroduced for adhesion and annealing purposes. After the soft bake, the wafer is ready to be exposed.

\section{Exposure}

There are numerous exposure methods that have been used in the semiconductor industry, but for the purposes of this discussion, only the most common will be mentioned. The vast majority of circuit manufacturing proceeds by exposing the resist 
through a mask. A mask is simply a glass or quartz plate that has been patterned with an opaque substance. The pattern on the mask is the image that will be transferred to the final wafer. Because extremely high precision is required in masks, they are typically created via electron-beam lithography. Electron-beam lithography uses an electron beam of a few nanometers in diameter to write directly onto the surface. It is a technique well suited to create high quality images on substrates, but because it is expensive, it is only used for fabrication of masks and a few other specialty applications.

Other important requirements for exposure masks are that they must be transparent to the desired wavelengths, exhibit dimensional stability and have a very low defect density. The cost of these masks is a very significant, accounting for nearly onethird of the total wafer processing costs [1],[24]. However, because of their robust nature the masks can be used over and over again to expose numerous wafers.

Once a mask is constructed, it is placed over the substrate to be patterned. There are three primary methods of exposure, each of which will be discussed briefly. In the simplest method, contact printing, the mask is placed directly onto the substrate, covering the entire wafer. The apparatus must allow precise adjustment of the vertical and horizontal positioning of both the wafer and mask to allow proper alignment. Once the wafer is aligned, the shutter is opened and the wafer is exposed to the radiation through the transparent areas of the mask. The main disadvantage of this technique is that the repeated contact between the mask and wafers gives rise to a large number of defects which result in a shortened mask life. [23] Contact printing was used for the deep UV exposure experiments detailed in Chapter 4. 
An alternate exposure technique, proximity printing, involves positioning the mask just above the surface of the wafer, with a small gap $(\sim 10 \mu \mathrm{m})$ separating the two. This gap eliminates the defects caused by direct contact with the wafer, but it increases diffraction effects and thus limits the minimum feature size that can be printed.

The final method, which is also the most commonly used, is projection printing. In projection printing, the mask is placed several centimeters away from the wafer and a system of lenses is used to focus the light through the mask and onto the substrate. In early versions of this method, the entire mask was focused onto the wafer at once [25]. To increase resolution, modern exposure systems use a step-and-repeat exposure. In these systems, the mask is only used to pattern a small portion of the wafer at once. After exposure, the wafer is mechanically stepped a specified distance, realigned and the image is projected again (Figure 1.2). This method ensures a long mask life and results in high resolution over the entire wafer surface. The $193 \mathrm{~nm}$ images shown in Chapter 5 were formed via this method.

The type of light source used will depend upon the photoresist type and the desired resolution. As feature sizes have continued to shrink, smaller and smaller wavelengths have been used to expose the resist films. Common near-UV systems utilize G-line $(436 \mathrm{~nm})$ and I-line $(365 \mathrm{~nm})$ light sources. Such systems are the most widely used in the semiconductor industry because of the maturity of the light sources, exposure systems, and photoresist chemistry. However, as feature sizes decrease further, mid-UV (250-300 nm) and deep-UV (190-250 nm) wavelengths became necessary. Current semiconductor research focuses on wavelengths of $157 \mathrm{~nm}$ and below. 
Thus far, it has been stated that photoresists will undergo one of many possible reactions (Table 1.1). It is necessary now to distinguish between the two primary classes of photoresists. Positive resists are photoresists that become more soluble in the developer upon exposure (Figure 1.1). These resists typically undergo chain scission or have inhibitors that are rendered inactive when exposed. The use of such resists results in a wafer image that is identical to that on the mask. Photoresists that become less soluble in the developer upon exposure are termed negative resists because the resultant wafer image is opposite that of the mask. These resists may have end-groups changed or may cross-link.

Post-Exposure Bake (PEB)

After the wafer has been exposed, it typically undergoes a post-exposure bake at $100{ }^{\circ} \mathrm{C}-120^{\circ} \mathrm{C}$. This bake facilitates the chemistry done by the newly cleaved PAG, and is thought to reduce standing wave effects caused by varied light penetration at different film depths. It is believed that this bake causes the photoactive compounds to diffuse throughout the film, reducing non-uniformities [26]. Because the glass transition temperatures $\left(\mathrm{T}_{\mathrm{g}}\right)$ of the photoresists used in this work $\left(\sim 40-50^{\circ} \mathrm{C}\right)$ were much lower than conventional polymers, a variety of PEB temperatures were used, including some below $100^{\circ} \mathrm{C}$. 


\section{Development}

After a wafer is exposed and the photoresist is altered such that solubility differences are created, the soluble regions of photoresist must be selectively removed without dissolving the desired resist features [27]. This process of removal is called development. There are three commonly used methods of development, each of which will be discussed in turn.

\section{Spray Developing}

In spray developing, an ultrasonic spray nozzle disperses the developing solution into small droplets which are then carried onto the wafer surface by a gas such as nitrogen [28]. The ultrasonic nozzle is often mounted on a motorized arm which oscillates the spray envelope of the developing solution from the center to the edge of the wafer [29]. The wafer is spun as the developing solution sprays onto the surface. The speed at which the wafer is spun must be optimized to insure that the developer is not stagnating on the surface, however, it must not be so fast that the developer is flung off before it has a chance to dissolve enough photoresists. The spent developer is often collected by the exhaust air flow to avoid contamination of subsequent wafers.

\section{Puddle Developing}

The puddle developing process begins with a short-duration dispense of developing solution onto a wafer spun at high velocity. The wafer is then gradually decelerated to a rotational speed of zero while chemical dispense continues. Eventually a puddle of developer is formed on the top of the substrate, held in place by surface 
tension. It is critical to time the dispensing arm (a movable nozzle used to apply the solution) movement to ensure that the entire wafer is exposed to developer for the same length of time. As the developer remains on the surface of the wafer, the altered photoresist is dissolved. An air flow exhaust is often introduced to minimize local differences created by evaporative cooling during rotation. In addition, midway through the dwell period, the wafer is slowly rotated one full rotation to gently agitate the puddle and distribute any air bubbles that may have formed during the dispensation of the developer. After the developer has dwelled on the surface for the desired time, a deionized water rinse displaces the developer and halts polymer dissolution. An excess volume of water is used to ensure complete removal of the developer solution. Finally, the wafer is slowly accelerated to a high speed to remove the residual liquid from the surface. Puddle developing is typically superior to spray developing. For most systems, it results in more uniform development, has fewer defects, and requires less expensive equipment than spray developing.

Both puddle and spray developing are feasible in liquid $\mathrm{CO}_{2}$ only under restricted conditions. Both techniques require a liquid/vapor interface. Thus, $\mathrm{scCO}_{2}$ is not suited to these applications. Puddle and spray developing may only be used to remove polymer regions that are soluble in $\mathrm{CO}_{2}$ in the liquid phase. A development technique which may use both $\mathrm{scCO}_{2}$ and liquid $\mathrm{CO}_{2}$ is immersion developing.

\section{Immersion Developing}

As may be surmised from its name, conventional immersion developing simply involves immersing a batch of exposed wafers in the developer solution. This process 
has the advantage of being able to process a large amount of wafers concurrently. It also does not require the expensive specialized equipment used in other development schemes. A variant of immersion developing in which a small amount of shear flow is introduced to the system is the technique used in this work.

\section{Developing Solutions}

A variety of different developers are used depending upon the photoresist system. In the past, alkali bases were often the developer of choice. Because of concerns about alkali metal contamination, the recent trend in developers has been towards non-metallic bases such as tetramethylammonium hydroxide [30].

\section{Photoresist Characterization}

As can be expected with any physical process, the actual dissolution of photoresist is seldom ideal. Taking the example of a positive resist, even at zero exposure there is still a small amount of the resist that will dissolve [31]. As the exposure energy increases, the film solubility increases until it can be entirely removed using the developer. In general, the sensitivity of a resist is defined as the minimum light energy that is needed to produce a good image on the film. The incident light energy is defined as

$$
\mathrm{E}=\mathrm{It},
$$

where $I$ is the light intensity and $t$ is the exposure time. Clearly, the light intensity will decrease as the light passes through the film. This intensity decrease follows LambertBeer's relation, 


$$
\mathrm{I}(\mathrm{z})=\mathrm{I}_{\mathrm{o}} \mathrm{e}^{-\alpha \mathrm{z}}
$$

where $\mathrm{I}(\mathrm{z})$ is the intensity of light in the z-direction, $\mathrm{I}_{\mathrm{o}}$ the intensity of light at the surface of the resist, and $\alpha$ is the absorption coefficient, usually assumed to be proportional to the concentration of light-absorbing species in the resist. As equation 1.4 suggests, it is necessary to use thinner resist films as the intensity of the light source drops, e.g., in 193 nm lithography.

A high sensitivity resist is desirable because it requires less exposure time and increases wafer throughput. In addition, a high sensitivity resist can often be used for shorter UV wavelengths where the light intensity is often lower [32]. The dependence of the sensitivity upon the wavelength of the radiation is typically presented in the form of a spectral response curve. A typical curve is given in Figure 1.3, where the fraction of resist remaining is plotted versus the incident energy at the surface. $\mathrm{E}_{\mathrm{T}}$ represents the threshold energy, below which the resist is nearly unchanged and $\mathrm{E}_{\mathrm{F}}$ is the energy at which solubility of the resists has been fully altered.

Another important parameter of polymer resist characterization is its contrast, $\gamma$, which is a measure of the sharpness of the transition from exposure to non-exposure. It may be evaluated from the slopes of the linear portions of the curves in Figure 1.3. For a positive resist, it is defined as,

$$
\gamma=\frac{1}{\log _{10} \frac{E_{T}}{E_{F}}} .
$$

The equation for a negative resist such as those used in this work is very similar, 


$$
\gamma=\frac{1}{\log _{10} \frac{E_{F}}{E_{T}}} .
$$

Resist contrast affects the image resolution. A high contrast is required to produce sharp features on the wafer. This is one of the most important parameters of any photoresist due in large part to the non-ideal exposure of the material. Due to the diffraction of light through small apertures (such as those on a photomask) the incident radiation on a wafer surface is not a step function, but is rather a smooth, continuous distribution of radiation with peaks and valleys. In order to translate these sloping gradients into sharp, square features, it is necessary to have as sharp a resist contrast as possible.

\section{Post Exposure Treatment}

\section{Hard Bake}

After conventional developing, the wafer is baked at $120^{\circ} \mathrm{C}-140{ }^{\circ} \mathrm{C}$ for several minutes. This removes any residual developer from the polymer resist. In addition, it is often thought to induce crosslinking in the resist, making the image more thermally stable and more resistant to the subsequent etching process [33]. When using a highly volatile solvent such as $\mathrm{CO}_{2}$, it was not be necessary to drive off any residual solvent, thus this step was eliminated. Because most of the work in this thesis concerns features in photoresist only, and not etched features in the silicon, a hard bake was not generally used. Such a processing step only became necessary during the brief etch resistance 
studies in which some of the films were spun from organic solvents, as is discussed in a later chapter.

\section{Etching}

Etching is the selective removal of material from the substrate. An etched pattern may be formed by either an additive or subtractive process. In the subtractive process, a film is first deposited or grown on the surface and then coated with a layer of photoresist. The desired pattern is transferred to the resist layer using lithography. During the subsequent etching step, the region covered by the resist is protected. Thus, when the resist is removed in the final stripping, the patterned underlayer remains.

In the additive method, the resist is first coated and defined before the desired film is deposited. The deposited film forms either on top of the resist or directly on the substrate surface where there are gaps in the resist pattern. The resist may then be stripped away, taking with it the unwanted film and leaving the desired features. This process is commonly referred to as lift-off [23].

\section{Etching Terminology}

The rate of film removal per unit time is called the etch rate. Also of importance is the selectivity of an etchant. The selectivity is defined as the ratio of the etch rate of one material to the etch rate of another. To obtain good contrast it is clearly desirable to have a high etch selectivity.

It is also necessary to consider the direction of film etching. When the etching proceeds at the same rate in all directions, it is termed isotropic. When the etch rate is 
much higher in one direction than another, it is said to be anisotropic. In addition, there may be different rates of lateral etching depending on film position. In general, the film typically is removed slightly slower near the substrate surface as shown in Figure 1.4. The distance $\mathrm{y}$ in Figure 1.4 is referred to as undercut, and the $\mathrm{x}$ dimension is called the etch bias. Ironically, it is usually desirable to overetch in order to ensure complete removal of the film and to create nearly vertical sidewalls (Figure 1.5).

The differences between isotropic and anisotropic etching are illustrated in Figure 1.6. Anisotropic etching results in much more vertical features but often leaves excess material on the sidewalls.

\section{Wet Etching}

In wet etching, a wafer or batch of wafers is simply immersed in a reactive solution. Etching of silicon-dioxide $\left(\mathrm{SiO}_{2}\right)$ is often carried out by immersing wafers in a dilute solution of hydroflouric acid (HF) buffered with ammonium fluoride $\left(\mathrm{NH}_{4} \mathrm{~F}\right)$. The process is isotropic and converts $\mathrm{SiO}_{2}$ into $\mathrm{H}_{2} \mathrm{SiF}_{6}$ which is dissolved and removed from the surface [34]. Wet etching may also be performed by spray rather than immersion. It is an effective technique for removing many materials. However, wet etching has been replaced by dry etching techniques in most semiconductor manufacturing lines.

\section{Dry Etching}

There are numerous dry etching techniques, most of which utilize some sort of high energy plasma to remove the desired material from the substrate. The choice of plasma chemistry will vary depending upon the application. Common etchants are 
fluorine-based plasmas $\left(\mathrm{CF}_{4} / \mathrm{O}_{2}\right.$ and $\left.\mathrm{SF}_{6} / \mathrm{O}_{2}\right)$, chlorine plasmas $\left(\mathrm{Cl}_{2}\right.$ and $\left.\mathrm{CCl}_{4} / \mathrm{O}_{2}\right)$, and bromine-containing plasmas. Each etchant has a different etch rate and selectivity that make it ideally suited for removing specific materials [35].

\section{Resist Stripping}

After the wafer has been etched and the desired pattern has been formed on the substrate, the photoresist mask must be removed. The final removal of the resist is called stripping. Like etching, there are a variety of stripping techniques. Wet stripping utilizes a powerful liquid solvent such as trichloroethylene or methyl-ethyl ketone to remove the polymer resist. Alternate techniques include plasma ashing, in which an oxygen plasma is used to convert organic materials to the volatile products $\mathrm{CO}, \mathrm{CO}_{2}$, and $\mathrm{H}_{2} \mathrm{O}$ [36]. Plasma ashing is also a useful technique for removing small amounts of photoresist between processing steps. When applied in this manner, it is referred to as descumming. In this research, stripping was all done using high pressure supercritical $\mathrm{CO}_{2}$ using a custom flow through apparatus.

With this brief introduction to conventional photolithographic process completed, the next chapter will describe the potential advantages of using liquid and supercritical $\mathrm{CO}_{2}$ as the primary solvent in the lithographic steps described above, completely replacing the aqueous and organic solvents currently used.

\section{References}


1. Moreau, W., et al. The Shot Size Reduction of Photoresist Formulatiosn. in Society of Photo-Optical Instrumentation Engineering. 1997: The International Sociert for Optical Engineering.

2. Wetmore, P.M., Supercritical Fluid Technology for Photoresist Developing, in Chemical Engineering. 1997, University of Massachusetts: Lowell.

3. DeSimone, J.M., Z. Guan, and C.S. Elsbernd, Synthesis of Fluoropolymers in Supercritical Carbon Dioxide. Science, 1992. 257: p. 945-947.

4. Allen, R.D. and G.M. Wallraff. 1997: U.S.

5. Ober, C.K., et al., Imaging Polymers with Supercritical Carbon Dioxide. Advanced Materials, 1997. 9(13): p. 1039-1043.

6. Gallagher-Wetmore, P., G.M. Wallraff, and R.D. Allen. Supercritical Fluid Processing: A New Dry Technique for Photoresist Developing. in The International Society for Optical Engineering. 1995.

7. Gabor, A.H., et al. Block and Random Copolymer Resists Designed for $193 \mathrm{~nm}$ Lithography and Environmentally Friendly Supercritical $\mathrm{CO}_{2}$ Development. 1997.

8. Moraeu, W.M., Semi-Conductor Lithography. 1988, New York: Plenum Press.

9. Bornside, D.E., C.W. Macosko, and L.E. Scriven, Spin Coating of a PMMA/Chlorobenze Solution. J. Electrochem. Soc., 1991. 138(1).

10. Rehg, T.J. and B.G. Higgins, Analysis of Solvent Evaporation Effects in Spin Coating of Colloidal Oxide Suspensions. 1989, University of California Davis. 
11. Larson, R.G. and T.J. Rehg, Spin Coating, in Liquid Film Coating, S.F. Kistler and P.M. Schweizer, Editors. 1997, CHapman \& Hall: London.

12. Parodi, M., et al., Spin Coating and Alternative Techniques for Flat Panel Displays. Semiconductor International, 1996: p. 101-105.

13. Emslie, A.G., F.T. Bonnner, and L.G. Peck, Flow of a Viscous Liquid on a Rotating Disk. J. Appl. Phys., 1957. 29(5).

14. Meyerhofer, D., Characteristics of Resist Films Produced by Spinning. J. Appl. Phys., 1978. 49(7).

15. Bornside, D.E., C.W. Macosko, and L.E. Scriven, Spin Coating: OneDimensional Model. J. Appl. Phys., 1989. 66(11): p. 5185-5193.

16. Yonkoski, R.K. and D.S. Soane, Model for Spin Coating in Microelectronic Applications. J. Appl. Phys., 1992. 72(2).

17. Birnie, D.P., Combined Flow and Evaporation During Spin Coating of Complex Solutions. Journal of Non-Crystalline Solids, 1997. 2118: p. 174-178.

18. Paul, S., et al., An Anthracene-Containing PMMA Derivative for Photoresist and Channel Waveguide Applications. Thin Solid Films, 1996. 288: p. 150-154.

19. Willson, C.G., R.A. Dammel, and A. Reiser. Photoresist Materials: A Historical Perspective. in Optical Microlithography X. 1994. Santa Clara, CA.

20. Levinson, H.J., Principles of Lithography. 2001, Bellinghman, Washington: SPIE Press. 
21. Ito, H., C.G. Wilsin, and J.M.J. Frechet, New UV Resists with Negative or Positive Tone. Digest of Technical Papers on the Symposium on VLSI Technology, 1982: p. 86-87.

22. Ito, H., Chemical Amplification Resists: History and Development within IBM. IBM J. Res. Develop., 1997. 41(1): p. 69-80.

23. El-Kareh, B., Fundamentals of Semiconductor Processing Technologies. 1995, Boston: Kluwer.

24. Levenson, M.D., Deep Pockets Needed in the Photoresist Industry. Solid State Technology, 1995: p. 32-40.

25. Hess, D.W. and K.F. Jensen, Microelectronics Processing. 1989, Washington, DC: American Chemical Society.

26. Turner, S.R. and R.C. Daly, The Chemistry of Photoresists, in Photopolymerization and Photoimaging Science and Technology, N.S. Allen, Editor. 1989, Elsevier Applied Science: New York.

27. Yada, Y., et al., Thin-Film Formation by Spin Coating: Characteristics of a Positive Photoresist. J. Appl. Phys., 1995. 34(11): p. 6279-6284.

28. Bokelberg, E. and J. Goetz. Manufacturing Requirements for a Single-Wafer Develop Process. in The International Society for Optical Engineering. 1995.

29. Zant, P.V., Microchip Fabrication. 2nd ed. 1990, New York: McGraw Hill. 209215.

30. White, L., Positive-Resist Processing for Step-and-Repeat Optical Lithography. RCA Review, 1986. 47: p. 345-379. 
31. McGillis, D.A., Lithography, in VLSI Technology, S.M. Sze, Editor. 1983, McGraw Hill: New York. p. 267.

32. Robbin, H. and B. Schwartz, Chemical Etching of Silicon, II. The System HF, $\mathrm{HNO}_{3}$ and $\mathrm{H}_{2} \mathrm{O}$. J. Electrochem. Soc., 1960. 106: p. 505.

33. Hawkins, D.T., Ion Milling (Ion Beam Etching). J. Vac. Sci. Technology, 1979. 16: p. 1051

34. Judge, J.S., A Study of the Dissolution of $\mathrm{SiO}_{2}$ in Acidic Fluorine Solutions. J. Electrochem. Soc., 1971. 118: p. 1772.

35. Flamm, D.L., Introduction to Plasma Chemistry, in Plasma Etching, D.M. Manos and D.L. Flamm, Editors. 1989, Academic Press: New York. p. 91.

36. Bernacki, S.E. and B.B. Kisicki, Controlled Film Formation During $\mathrm{CCl}_{4}$ Plasma Etching. J. Electrochem. Soc., 1984. 131(8): p. 1926-1931. 


\section{Photolithography}
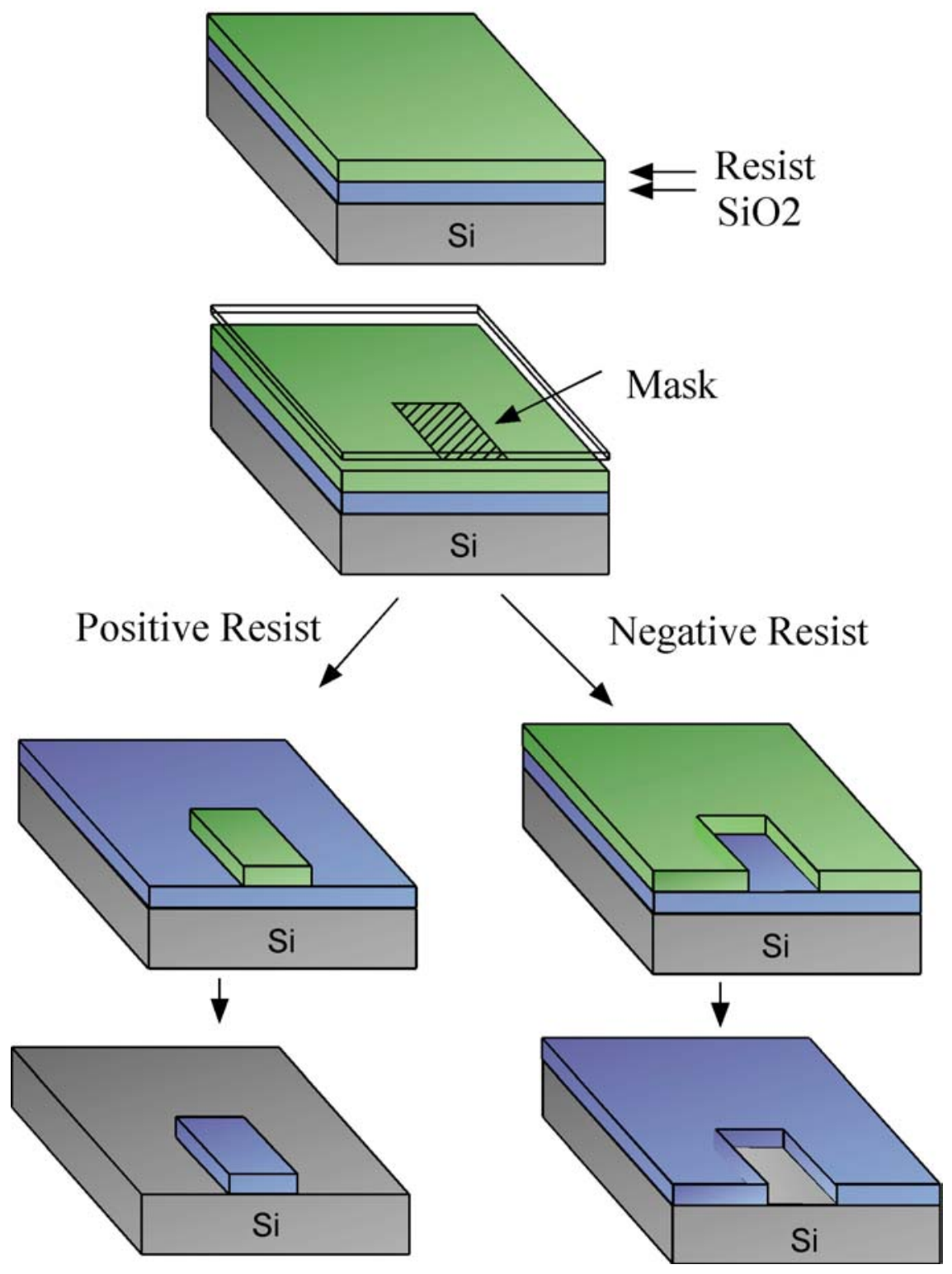

Figure 1.1. Positive and negative resist exposure and development. 


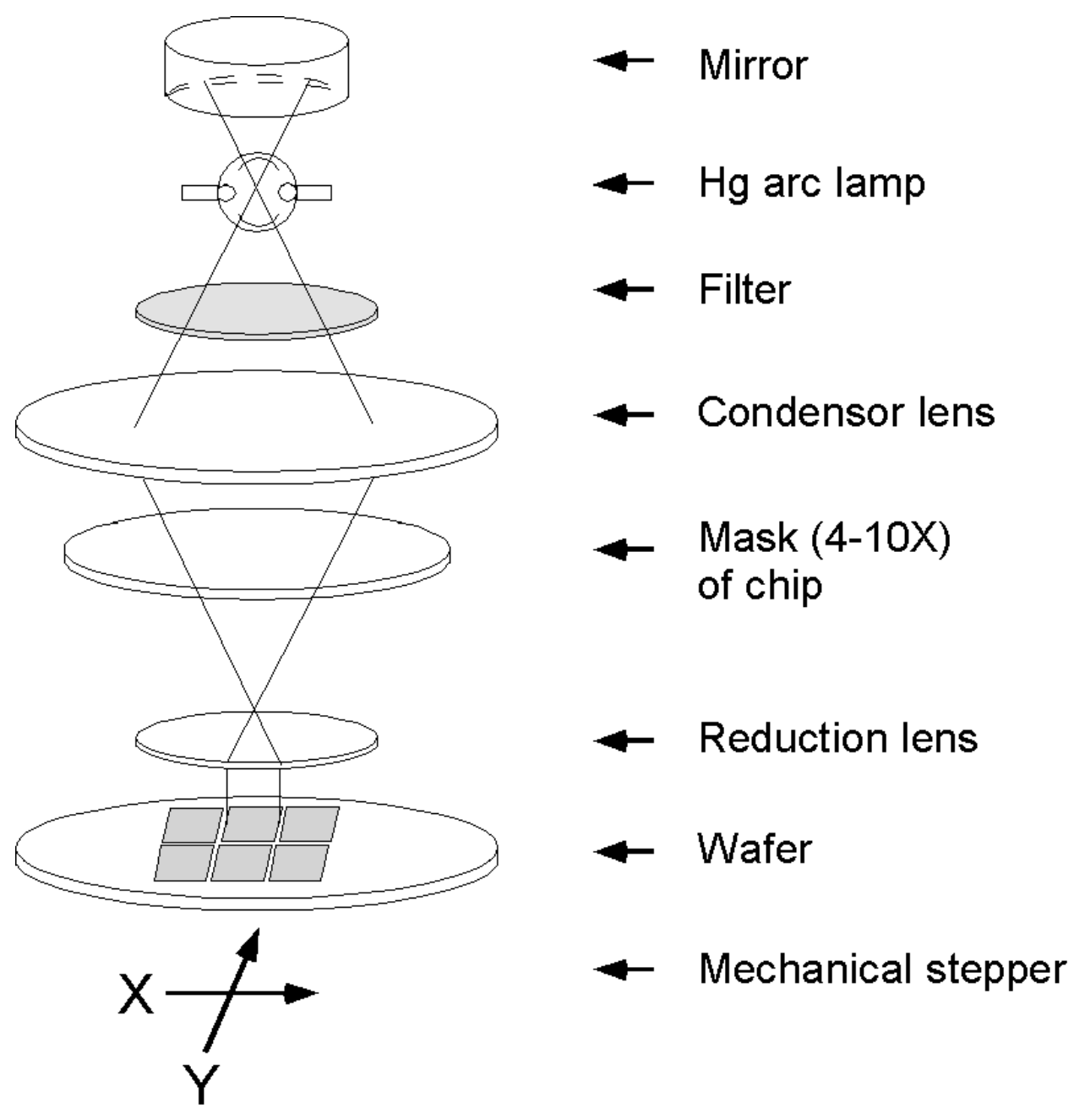

Figure 1.2. Schematic of a reduction step-and-repeat projection system 


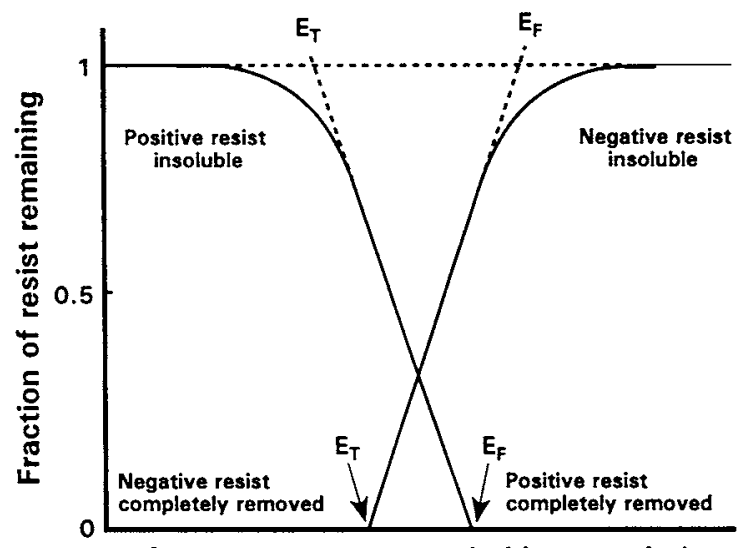

Log exposure energy (arbitrary units)

(Scales differ for positive and negative resist)

Figure 1.3. Sensitivity plot of positive and negative photoresists. 


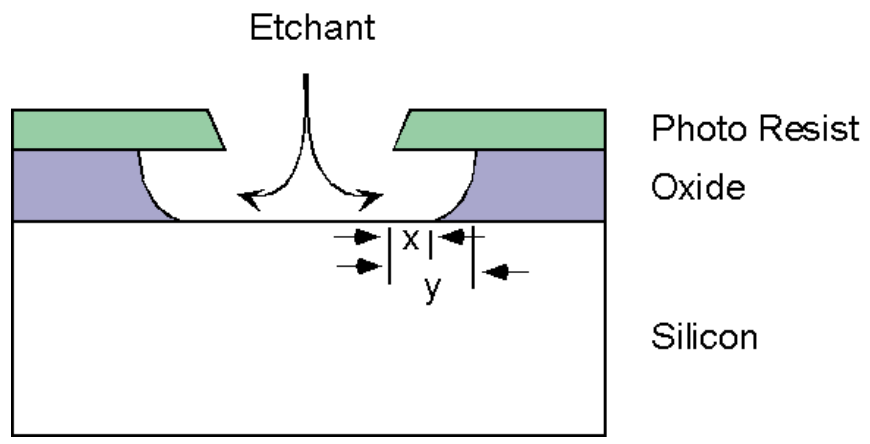

Figure 1.4. Lateral etch dimensions. 


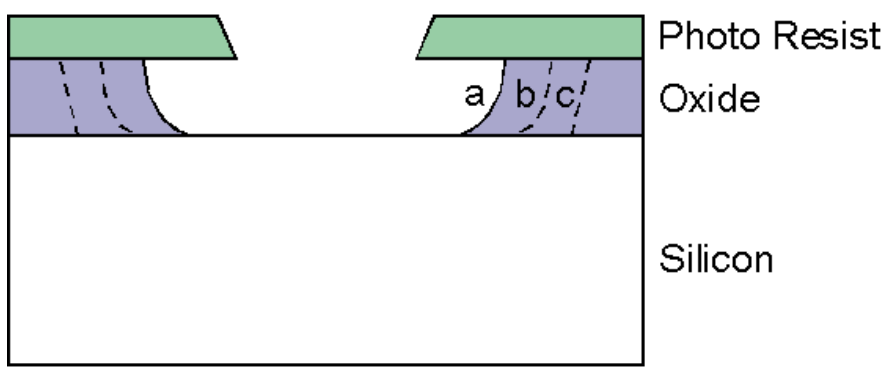

Figure 1.5. Illustration of overetching on undercutting and sidewall slope: (a) no overetch, (b) moderate overetch, (c) long overetch. 


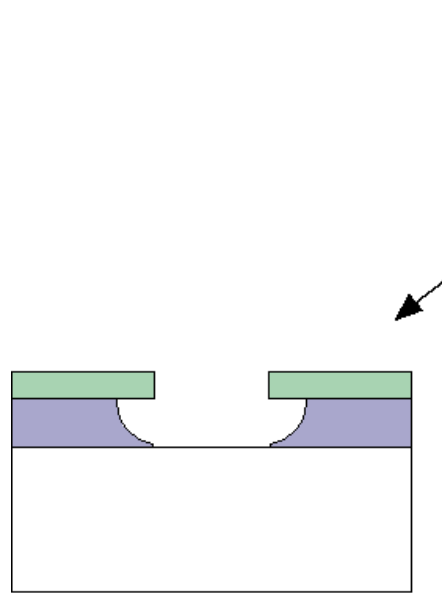

(a)

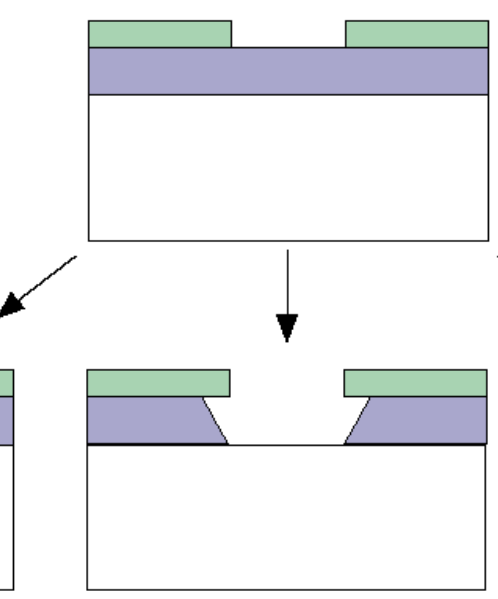

(b)
Photoresist

Oxide

Substrate
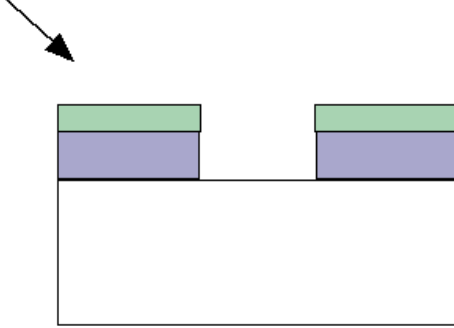

(c)

Figure 1.6. Etch directionality: (a) isotropic etch, (b) moderately anisotropic etch, (c) anisotropic etch. 


\section{Chapter 2. Applicability of $\mathrm{CO}_{2}$ to Photolithography}

Dense phase carbon dioxide $\left(\mathrm{CO}_{2}\right)$ is well suited to replace conventional solvents in spin casting, image development, and photoresist stripping, resulting in a completely "dry" lithographic process. While the shift from a wet solvent-based system to one utilizing a compressed phase solvent such as $\mathrm{CO}_{2}$ may seem daunting, there are enormous potential benefits. Also, such a change is not without precedent; in recent years many $\mathrm{CO}_{2}$ based processes have proven their worth in other industries. $\mathrm{CO}_{2}$ has proven its value as an alternative solvent for a variety of industrial extraction processes[1], polymer processing [2], consumer $\mathrm{CO}_{2}$-based garment cleaning[3], metal degreasing (Appendix C), and

polymer synthesis including a state-of-the art Teflon ${ }^{\circledR}$ manufacturing plant built by Dupont in North Carolina [4], [5], [6].

Dense phase $\mathrm{CO}_{2}$ possesses several unique properties that make it appealing as a solvent for lithography. $\mathrm{CO}_{2}$ in the liquid and supercritical states has a density only slightly less than that of water [7]. Because of this high density, it is possible to dissolve relatively large concentrations of polymers and other materials in $\mathrm{CO}_{2}$. When using resist systems designed for $\mathrm{CO}_{2}$, it is not difficult to achieve solids concentrations of $25 \mathrm{wt} \%$ or more. However, despite its relatively large density, dense phase $\mathrm{CO}_{2}$ has a very low viscosity $\left(5.7 \times 10^{-6} \mathrm{~Pa}^{*} \mathrm{~s}\right.$ at 25 degrees $\mathrm{C}$ and $\left.930 \mathrm{psi}\right)$. This low viscosity allows one to coat very thin films using $\mathrm{CO}_{2}$. In addition, the low viscosity allows $\mathrm{CO}_{2}$ to penetrate into small crevices and features, much as a gas would, but its high density gives it solubility properties similar to conventional liquids, allowing $\mathrm{CO}_{2}$ to remove large 
amounts of resist from tiny crevices. This combination of high density and low viscosity make $\mathrm{CO}_{2}$ well suited to film removal, and to deposition of thin films over large substrates. This should prove an asset as wafer sizes increase to $300 \mathrm{~mm}$ and beyond.

One of the major hurdles to successful $157 \mathrm{~nm}$ lithography is the opacity of conventional resist systems to $157 \mathrm{~nm}$ radiation[8]. Resists with a high optical density will not allow sufficient penetration of the radiation, and thus the photochemistry is inhibited. However, Kunz et al.[9], among others, found that while most polymeric materials have a high optical absorbance at $157 \mathrm{~nm}$, fluoropolymers, hydrofluoropolymers and siloxane polymers are relatively transparent. Thus, the majority of $157 \mathrm{~nm}$ resists use chemistry based on these polymers. And while many of these materials exhibit poor solubility in conventional organic solvents, there exists a large body of literature showing that such materials often have high solubilities in dense phase $\mathrm{CO}_{2}$ [4]. In fact, when such polymers are designed with $\mathrm{CO}_{2}$-solubility in mind, it is possible to form solutions under relatively mild conditions, e.g., liquid $\mathrm{CO}_{2}$.

Another source of difficulty in the fabrication of very small features is that of image collapse. As feature sizes continue to shrink faster than resist film thicknesses, high aspect ratio features are created. After developing such features in aqueous solutions, the subsequent drying steps often expose these features to significant lateral stress. This stress is caused by the surface tension of the evaporating aqueous solution, and often leads to feature collapse, thus destroying the image [10]. Correcting this problem is often difficult because the high surface tension is an inherent property of water. However, $\mathrm{CO}_{2}$ does not suffer this same shortcoming. $\mathrm{CO}_{2}$ has a much lower 
surface tension than water (see Figure 2.1), and, indeed, as the density of the liquid and vapor phases of $\mathrm{CO}_{2}$ converge and the fluid enters the supercritical state, the surface tension forces disappear completely. In such a state, the fluid exerts no lateral forces to distort the image. Recognizing this potential, Namatsu, et al. have shown that it is possible to use supercritical $\mathrm{CO}_{2}$ to physically displace the aqueous developing fluid using a multi-step process to eliminate feature collapse even for very high aspect ratio features [11].

In any discussion of the numerous technical advantages of using $\mathrm{CO}_{2}$, we should be remiss if we failed to mention the significant environmental benefits of using $\mathrm{CO}_{2}$. Unlike many of the conventional solvents used in lithography, $\mathrm{CO}_{2}$ is neither harmful to those working in close proximity to it, nor to the larger environment [12]. This is by no means insignificant. In conventional lithography, wafers often undergo the lithographic process 25 or more times to build up the requisite layers of circuitry and interconnects, thus generating large quantities of waste. In addition, semiconductor fabrication facilities often consume enormous quantities of water per day. The 1997 Semiconductor Industry Association (SIA) National Technology Roadmap for Semiconductors (NTRS) (and its 1999 update) and the 1996 Electronics Industry Environmental Roadmap (EEIR) both emphasize the importance of reducing wet organic and aqueous consumption (and in turn waste streams) in the industry. Completely replacing the conventional solvents with $\mathrm{CO}_{2}$ would clearly meet this goal. And since $\mathrm{CO}_{2}$ is a byproduct of many other industrial reactions, and is also a recyclable material, no additional $\mathrm{CO}_{2}$ need be generated nor released into the environment. These advantages, as well as many others listed in Table 
2.1, warrant the investigation of $\mathrm{CO}_{2}$ spin coating as an alternative to conventional spin coating systems. 
Table 2.1. Potential advantages and disadvantages of $\mathrm{CO}_{2}$ based spin coating compared to conventional methods.

\begin{tabular}{|c|c|}
\hline Conventional Spin Coating & Liquid $\mathrm{CO}_{2}$-based Spin Coating \\
\hline $\begin{array}{l}\text { Evaporation (a key process parameter) is } \\
\text { determined by the inherent vapor pressure of the } \\
\text { solvent used. }\end{array}$ & $\begin{array}{l}\text { Evaporation is controlled precisely by controlling } \\
\text { the pressure in the spin coating chamber, and may } \\
\text { be altered at will to tailor film properties. }\end{array}$ \\
\hline $\begin{array}{l}\text { After spin coating, a post apply bake is required for } \\
\text { film annealing and to drive off residual solvent. }\end{array}$ & $\begin{array}{l}\text { The gaseous nature of } \mathrm{CO}_{2} \text { potentially eliminates } \\
\text { the need for a baking step to drive off residual } \\
\text { solvent; film annealing may also prove unnecessary } \\
\text { due to the high plasticization of polymers exposed } \\
\text { to } \mathrm{CO}_{2} \text {. }\end{array}$ \\
\hline $\begin{array}{c}\text { Many conventional solvents are unsuitable for } \\
\text { spinning highly fluorinated, next generation } 157 \mathrm{~nm} \\
\text { resists. }\end{array}$ & $\begin{array}{c}\mathrm{CO}_{2} \text { is an excellent solvent for many fluorinated } \\
\text { polymers. }\end{array}$ \\
\hline $\begin{array}{l}\text { Conventional organic solvents have relatively high } \\
\text { viscosity, resulting in some difficulties when } \\
\text { spinning the larger } 300 \mathrm{~mm} \text { wafers. }\end{array}$ & $\begin{array}{l}\mathrm{CO}_{2} \text { has a low viscosity, potentially improving the } \\
\text { spinning of larger films. } \mathrm{CO}_{2} \text { may also reduce edge } \\
\text { beading because of its very low surface tension. }\end{array}$ \\
\hline $\begin{array}{l}\text { Most conventional systems require an additional } \\
\text { undercoat to promote film adhesion. }\end{array}$ & $\begin{array}{l}\text { Our work with } \mathrm{CO}_{2} \text { to date has shown excellent film } \\
\text { adhesion on pure silicon, potentially eliminating the } \\
\text { need for adhesion promoters as long as } \mathrm{CO}_{2} \text { is used } \\
\text { for both the spinning and development. }\end{array}$ \\
\hline $\begin{array}{l}\text { Surfactants often used to decrease surface tension } \\
\text { and improve wetting. }\end{array}$ & $\begin{array}{l}\text { Low surface tension leads to excellent wetting } \\
\text { without the use of surfactants or other additives. }\end{array}$ \\
\hline $\begin{array}{l}\text { Utilizes organic solvents, many of which are } \\
\text { harmful to the environment and workers. }\end{array}$ & Utilizes environmentally benign $\mathrm{CO}_{2}$. \\
\hline $\begin{array}{l}\text { Most operations conducted under atmospheric } \\
\text { pressure. }\end{array}$ & $\begin{array}{l}\text { Requires specialized, high-pressure equipment for } \\
\text { all steps using } \mathrm{CO}_{2} \text {. }\end{array}$ \\
\hline Uses proven technology. & $\begin{array}{l}\text { Requires a re-evaluation of current processes and } \\
\text { the complete replacement of both resist chemistry } \\
\text { and equipment. }\end{array}$ \\
\hline $\begin{array}{c}\text { Current processes are streamlined for high } \\
\text { throughput. }\end{array}$ & $\begin{array}{c}\mathrm{CO}_{2} \text { based processing requires several transitions } \\
\text { from high-pressure to atmospheric conditions, } \\
\text { potentially slowing output. }\end{array}$ \\
\hline
\end{tabular}


Despite the numerous advantages of using $\mathrm{CO}_{2}$, there are several hurdles as well. The design and operation of equipment under pressure is more challenging than conventional procedures. Also, while many fluoropolymers exhibit solubility in $\mathrm{CO}_{2}$, conventional photoresist materials are not sufficiently soluble for use in $\mathrm{CO}_{2}$-based spin coating, development, and stripping. Thus an integrated approach was required, synthesizing highly $\mathrm{CO}_{2}$-soluble photolithographic materials concurrently with the implementation of $\mathrm{CO}_{2}$-based lithographic equipment and procedures.

\section{$\mathrm{CO}_{2}$ Lithography - Concepts}

A natural question that arises is how does one use the same solvent to spin cast, develop, and strip a photoresist. All of these processes require solvents with different strengths, and if we were using conventional solvents, we would clearly have to use a different solvent for each step. However, one of the compelling reasons to use $\mathrm{CO}_{2}$ is the great tunability of the solvent, i.e., it is possible to drastically change the characteristics of $\mathrm{CO}_{2}$ by changing the process conditions. For example, in order to spin cast films of photoresist using $\mathrm{CO}_{2}$, it is necessary to evaporate the $\mathrm{CO}_{2}$ out of the solution and form a dry resist film. Thus, we must spin coat using conditions under which $\mathrm{CO}_{2}$ is a liquid that may then evaporate into a vapor atmosphere. However, upon initial investigation it was found that our polymer resins were not sufficiently soluble in $\mathrm{CO}_{2}$ liquid at ambient temperatures. Fortunately, $\mathrm{CO}_{2}$ density, and thus solvent strength, are highly dependent upon temperature. In Figure 2.1 it can be seen that as we lower the operating temperature 
$\sim 10{ }^{\circ} \mathrm{C}$, we increase the liquid density dramatically, thus allowing us to dissolve sufficient concentrations of resist into the $\mathrm{CO}_{2}$ solution.

A similar situation occurs during the development and stripping processes. When developing a negative tone resist, the developing solvent must be fairly mild, such that it does not remove the exposed portions of the film. However, when the etching is complete, it is then necessary to strip away the exposed resist as well. A highly tunable solvent such as $\mathrm{CO}_{2}$ can be used for both of these steps. All that is required is a change in the physical conditions. Figure 2.2 illustrates how this may be accomplished, showing the vast difference in $\mathrm{CO}_{2}$ density (and thus solvent strength) that may be obtained simply by changing the operating pressure and temperature.

This is perhaps better illustrated in Figure 2.3, which shows how all of the lithographic processes may be carried out using $\mathrm{CO}_{2}$, but at different conditions. In this figure, the grey areas indicate the solvent strengths needed for each process. After spin coating and development, it is possible to strip the same resist using $\mathrm{CO}_{2}$ as indicated. Thus, we may use one solvent to carry out three vastly different processes.

\section{$\mathrm{CO}_{2}$ Chemistry and Photoresist Design}

\section{Photoresist Chemistry}

Multiple characteristics are important when designing polymer photoresists. Resists are formulated to have good adhesion to the substrate, high selectivity, high contrast, and high resistance to etching. An even more basic requirement for the photoresists used in this research is that they be soluble in $\mathrm{CO}_{2}$ at vapor pressure, a non-trivial requirement. 
The nature of $\mathrm{CO}_{2}$ copolymer interactions is not currently well understood [13],[4],[12],[14]. Numerous researchers have investigated polymer systems in $\mathrm{CO}_{2}$. Beckman and co-workers have described the solubility of poly(perfluoropropylene oxide) and poly(dimethylsiloxane) in $\mathrm{CO}_{2}[15],[16]$. Krukonis has shown that poly(phenylmethylsilicone) and perfluoroalkyl polyethers are soluble as well [17]. Extensive work has been conducted by DeSimone and coworkers showing that fluorinated octyl acrylates are highly soluble in $\mathrm{CO}_{2}$ [18],[19]. Recently, several authors have attempted to quantify $\mathrm{CO}_{2}$ polymer interactions, focusing on intermolecular forces, dipole, quadrupole, and free volume effects [12], [14]. In general, they have found that $\mathrm{CO}_{2}$ is a poor solvent for both highly polar and highly nonpolar polymers. Fluorinated polymers typically exhibit high solubilities in $\mathrm{CO}_{2}$. Siloxanes are also somewhat soluble in $\mathrm{CO}_{2}$. While the solubility of some of the simpler copolymers has been explained, polymer solubility in $\mathrm{CO}_{2}$ remains largely an empirical study.

\section{Negative Resist}

The photoresists used in this work were synthesized by members of DeSimone's research group at UNC-Chapel Hill. Initial polymers were synthesized by Jonathan Kendall, with later polymerizations done by Chris McAdams and Devin Flowers. The majority of the materials discussed in this dissertation were the result of synthetic work done by Devin Flowers. Building upon the previous experience of the group [20],[18],[4]. , the first photoresists were based upon fluorinated octyl methacrylate copolymers. (1H,1H-perfluorooctyl)methacrylate (FOMA) was copolymerized with t- 
butyl methacrylate (TBM) using azobisisobutyronitrile (AIBN) as a free radical initiator.

It was carried out in a high pressure $\mathrm{CO}_{2}$ vessel under the conditions shown in Figure 2.4.

In a conventional organic solvent this copolymer would act as a positive resist, with the MAA acid (after cleavage) being much more soluble than the original THPMA in a solution of TMAH. However, in a low polarity solvent such as $\mathrm{CO}_{2}$ the transformation from the PFOMA- $r$-THPMA to a PFOMA-r-MAA results in a less $\mathrm{CO}_{2}-$ soluble material (Figure 2.5). This same trend is observed for many resists in $\mathrm{CO}_{2}$; materials that act as a negative tone resist in conventional organic solvents will behave as positive tone resists in $\mathrm{CO}_{2}$. This resin was found to be soluble in $\mathrm{CO}_{2}$ at relatively mild conditions, and similar resins have been shown to be developable in $\mathrm{CO}_{2}$ by Ober et al. [21]. Table 2.2 contains further details on the particular samples synthesized by Chris McAdams, including solubility data.

Table 2.2. Properties of Poly(FOMA-r-THPMA) resins used.

\begin{tabular}{|c|c|c|c|c|c|c|c|c|}
\hline \multirow{2}{*}{$\begin{array}{l}\text { Sample \# } \\
\text { CLM-A- }\end{array}$} & \multirow{2}{*}{$\begin{array}{c}\text { Target } \\
\text { MW }\end{array}$} & \multirow{2}{*}{$\begin{array}{c}\text { FOMA } \\
\text { Mole \% } \\
\text { (Feed) }\end{array}$} & \multirow{2}{*}{$\begin{array}{l}\text { FOMA } \\
\text { Mole \% } \\
\text { (NMR) }\end{array}$} & \multirow{2}{*}{$\begin{array}{c}T_{d} \\
\text { (TGA) } \\
\text { onset }\end{array}$} & \multicolumn{4}{|c|}{ Cloud Point (psi at $1 \%$ w/v) } \\
\hline & & & & & $10^{\circ} \mathrm{C}$ & $25^{\circ} \mathrm{C}$ & $35^{\circ} \mathrm{C}$ & $45^{\circ} \mathrm{C}$ \\
\hline 001 & $15 \mathrm{~K}$ & 80 & 80 & 155.8 & sol. & 1350 & 1800 & 2400 \\
\hline 003 & $5 \mathrm{~K}$ & 65 & 66 & 154.5 & sol. & 1400 & 2000 & 2450 \\
\hline 005 & $15 \mathrm{~K}$ & 95 & 94 & 169.7 & - & 1100 & 1650 & 2150 \\
\hline 007 & $5 \mathrm{~K}$ & 80 & 79 & 157.3 & - & 975 & 1550 & 2050 \\
\hline 009 & $25 \mathrm{~K}$ & 65 & 67 & 146.0 & 1075 & 1900 & 2350 & 3200 \\
\hline 011 & $25 \mathrm{~K}$ & 95 & 91 & 170.1 & - & 1200 & 1725 & 2250 \\
\hline 013 & $15 \mathrm{~K}$ & 65 & 65 & - & 750 & 1750 & 2300 & 2850 \\
\hline 015 & $25 \mathrm{~K}$ & 80 & 80 & - & sol. & 1450 & 2050 & 2550 \\
\hline 017 & $5 \mathrm{~K}$ & 95 & 95 & - & - & - & 1450 & 1925 \\
\hline
\end{tabular}

\section{Photoacid Generators}

In the course of this work, chemists and UNC synthesized a variety of potential photoacid generators, many of which were found to be insoluble in $\mathrm{CO}_{2}$ at the conditions needed. 
Further efforts by Devin Flowers yielded two usable PAGs, diphenyliodonium tert-(3,5bis(trifluoromethyl) phenyl boronium and 2-perfluorohexyl-6-nitrobenzyl tosylate (structures shown in Figure 2.5). Both of these PAGs were found to be soluble under liquid $\mathrm{CO}_{2}$ conditions at sub-ambient temperatures. Their absorbance spectra and respective advantages are discussed in Chapter 4.

\section{Spin Coating with Liquid $\mathrm{CO}_{2}$}

\section{Solubility Issues}

As mentioned previously, spin coating involves dissolving a solute in a liquid that is then spun on the desired substrate. Although more polymers are soluble in $\mathrm{scCO}_{2}$ than in liquid $\mathrm{CO}_{2}, \mathrm{scCO}_{2}$ is not a suitable medium for spin coating as there is no meniscus in a supercritical fluid. Any photoresist used in the process must be soluble in liquid $\mathrm{CO}_{2}$. As discussed previously, the density and solvent power of $\mathrm{CO}_{2}$ can be altered by changing the pressure of the fluid. However, by restricting the phase to liquid $\mathrm{CO}_{2}$, the pressure is determined by the vapor pressure of the liquid at the operating temperature. Fortunately, the solvent strength of $\mathrm{CO}_{2}$ is also highly dependent upon temperature. While solvent strength is affected by many factors, one of the most important is its direct dependence upon density. For the most part, as the density of the liquid $\mathrm{CO}_{2}$ increases, the polymer will become more soluble. The variation of liquid $\mathrm{CO}_{2}$ density versus temperature is given in Figure 2.6. As can be seen from this figure, a small decrease in temperature from $25^{\circ} \mathrm{C}$ to $5{ }^{\circ} \mathrm{C}$ increases the density of liquid $\mathrm{CO}_{2}$ almost $50 \%$. This significantly raises the solubility of the photoresist polymers in liquid $\mathrm{CO}_{2}$. 
The temperatures (and associated vapor pressures) necessary to dissolve various polymer samples are given in Table 2.3. The samples are random copolymers of PFOMA and TBM. Decreasing the temperature of the liquid $\mathrm{CO}_{2}$ down to $15^{\circ} \mathrm{C}$ results in the solubility of up to $40 \mathrm{wt} \%$ polymer. Thus, by cooling the spin coating apparatus down to sub-ambient temperatures, it is possible to use the high concentration solutions required for effective coating.

Table 2.3. Solubility of Polymer Resins in $\mathrm{CO}_{2}$

\begin{tabular}{|c|c|c|c|}
\hline $\begin{array}{l}\text { PFOMA-TBM } \\
\text { Sample Name }\end{array}$ & $\begin{array}{c}\text { Mol\% TBM in } \\
\text { polymer }\end{array}$ & $\begin{array}{c}\text { Wt\% of sample } \\
\text { in solution }\end{array}$ & $\begin{array}{c}\text { Cloud Point Temperature } \\
\text { (Vapor Pressure) }\end{array}$ \\
\hline \multirow[t]{2}{*}{ KLA1-15-1 } & 33 & 10 & $16.0^{\circ} \mathrm{C}(618 \mathrm{psi})$ \\
\hline & & 20 & $14.8^{\circ} \mathrm{C}(588 \mathrm{psi})$ \\
\hline \multirow[t]{2}{*}{ KLA1-10-1 } & 20 & 10 & $16.4^{\circ} \mathrm{C}(628 \mathrm{psi})$ \\
\hline & & 20 & $15.6^{\circ} \mathrm{C}(611 \mathrm{psi})$ \\
\hline \multirow[t]{3}{*}{ JLK1-75-1 } & 12 & 10 & $17.5^{\circ} \mathrm{C}(661 \mathrm{psi})$ \\
\hline & & 20 & $16.8^{\circ} \mathrm{C}(629 \mathrm{psi})$ \\
\hline & & 40 & $16.3^{\circ} \mathrm{C}(621 \mathrm{psi})$ \\
\hline \multirow[t]{2}{*}{ JLK1-71-1 } & 0 & 20 & $16.6^{\circ} \mathrm{C}(629 \mathrm{psi})$ \\
\hline & & 40 & $16.1^{\circ} \mathrm{C}(615 \mathrm{psi})$ \\
\hline
\end{tabular}




\section{Spin Coating Apparatus}

The first chamber in which the films were spun is a large, top-loading, pressure chamber (J. D. Clipper) constructed to withstand pressures up to $3000 \mathrm{psi}$. To achieve the necessary temperatures, the chamber and all of the associated equipment were placed in a large chromatography refrigeration cabinet (Revco) capable of maintaining the temperature from $0{ }^{\circ} \mathrm{C}-20^{\circ} \mathrm{C}$ (Figure 2.7). By regulating the temperature of the entire apparatus, it is possible to achieve a large range of liquid $\mathrm{CO}_{2}$ densities and vapor pressures.

To begin an experiment, a photoresist polymer was added to a $10 \mathrm{ml}$ high pressure view cell and filled with liquid $\mathrm{CO}_{2}$. The amount of polymer and $\mathrm{CO}_{2}$ is varied in order to obtain solution concentrations ranging from $5 \mathrm{wt} \%-25 \mathrm{wt} \%$ polymer. The dissolution rate of many of the photoresist polymers was slow, requiring the solution to be stirred overnight to achieve complete solubility. The polymer was deemed to be completely dissolved when the solution becomes visually transparent. Upon complete dissolution, the vapor pressure of the mixture was measured using a pressure transducer (Druck PMP 4010) accurate to +/- 0.3 psi and was displayed on the pressure indicator (DPI 282).

The transducer lines and inlets into the view cell are 1/16" tubing (High Pressure Inc.). Lines into the spinning chamber are $1 / 4 "$ high pressure tubing, with the exception 
of the solution inlet line which is $1 / 8^{\prime \prime}$ tubing. The smaller diameter is used to minimize the volume of the line. Initially, 1/16" tubing was used for the injection line, but it frequently became plugged if polymer dropped out of solution prematurely. Further refinements resulted in the installation of a 0.2 micron filter in the injection line to ensure solution purity.

After the solution is prepared, a substrate is cleaned with successive rinses of acetone, methanol, and DI water. The wafer is then placed onto the rotating chuck. Initial experiments were conducted with glass substrates (2" x 2", VWR). Later refinements allowed the use of an alternate wafer chuck for the spinning of $125 \mathrm{~mm}$ silicon wafers ( $<100>$ orientation, Boron doped, polished on one side, International Wafer Service). Further details on the chuck and chamber are given in Chapter 3.

\section{Measurement of Film Quality}

The first inspections of coated film quality were simple visual inspections. Such inspections were used to determine if the film covered the entire substrate and if any large scale defects were present. Measurements of film thickness and uniformity required additional techniques.

Films were first characterized using a precision profilometer (Tencor Instruments). A profilometer measures the height of any substrate with a precision stylus which moves along the surface while maintaining physical contact at all times. This technique yields a two-dimensional cross-section of the film. To obtain absolute film thicknesses and uniformities, it was necessary to remove the photoresist polymer from particular areas to provide a baseline for measurements. The polymer film was removed either by 
physically scraping the film or by dissolving a small portion of the film in a fluorinated solvent such as Freon-113 or trifluorotolunene.

Profilometry provides accurate film thicknesses over a small length. Ellipsometry, in which the film thickness is determined by the refraction of light, is used to verify profilometry measurements and provide more precise thickness values when required. In addition to ellipsometry, films were sometimes characterized using scanning electron microscopy (SEM) and atomic force microscopy (AFM).

\section{Falling Cylinder Viscometer}

It was necessary to determine values of the $\mathrm{CO}_{2}$-photoresist solution viscosity in order to predict film thicknesses and compare empirical results with theory. Viscosity measurements utilized a high-pressure falling cylinder viscometer [22],[23],[24]. The device consisted of a long high-pressure tube filled with the liquid $\mathrm{CO}_{2}$ / photoresist solution (Figure 2.8). The viscosity was determined by measuring the time it takes for a sinker (a small cylinder) to fall through a known distance of tubing [25],[26].

The motion of the falling sinker results in flow in the annular region between the viscometer tube and the sinker. If the system is designed with a minimal clearance between the sinker and the tube wall, the shear rate is nearly uniform and equal to the wall shear rate, $\gamma_{\mathrm{w}}$, and is given by [27],[28],

$$
\gamma_{w}=\frac{v_{\infty}}{(R-\kappa R)},
$$


where $v_{\infty}$ is the terminal velocity of the sinker and the radii are as given in Figure 2.9. The terminal velocity of the sinker is determined by the sinker density and fluid viscosity and density. Thus, it is difficult to obtain numerous shear rates with a given fluid [29]. In addition, if the same sinker is used with different fluids and pressures, direct comparison of the data will be somewhat inaccurate if the viscosities vary over a wide range. It is also difficult to maintain perfectly vertical motion of the sinker [30]. However, despite various problems, a falling-cylinder viscometer was found to be a quick and inexpensive method of obtaining estimates of the viscosity of the photoresist- $\mathrm{CO}_{2}$ solutions.

The viscosity of the solution is determined by measuring the time required for the sinker to fall a given distance. By making a force balance on the cylindrical sinker, an expression for the fluid viscosity is obtained [31],

$$
\eta=\frac{\left(\rho_{c}-\rho_{f}\right) g(\kappa R)^{2}}{2 v_{\infty}}\left(\operatorname{Ln} \frac{1}{\kappa}-\frac{1-\kappa^{2}}{1+\kappa^{2}}\right) .
$$

Here, $\rho_{\mathrm{c}}$ and $\rho_{\mathrm{f}}$ are the densities of the sinker and the fluid, respectively, and $\mathrm{g}$ is the acceleration due to gravity. For the case of a narrow clearance between the sinker and the tube walls, this expression reduces to,

$$
\eta=\frac{\left(\rho_{c}-\rho_{f}\right) g(\kappa R)^{2} \varepsilon^{3}}{6 v_{\infty}}\left(1-\frac{1}{2} \varepsilon-\frac{13}{20} \varepsilon^{2}+\ldots\right)
$$


where $\varepsilon=1-\kappa$. For a given viscometer, it is possible to lump those quantities which depend on the physical dimensions of the system into one constant. The viscosity is then proportional to the density difference between the sinker and solvent and to a calibration constant $\mathrm{C}$,

$$
\eta=C\left(\rho_{c}-\rho_{f}\right) t .
$$

The calibration constant of the viscometer is determined by conducting trials with fluids of known viscosity. The calibration constant can vary with the fluid Reynolds number, leading to errors when applied to turbulent flow.

The falling-cylinder viscometer used in this work consisted of a 3/8" O. D., 0.203" I.D. high-pressure tube with a small reservoir on the top and the bottom of the tube (see Figure 2.9). The system was filled with the desired photoresist solution, and then placed in a thermally isolated chamber to maintain the proper temperature. The velocity of the sinker was measured using small linear voltage transducers (LVDT) which registered the change in magnetic field as the sinker moved through the tube. The signal from the LVDTs was transferred to a computer data acquisition unit which stored values for various trials. Prior to investigating photoresist- $\mathrm{CO}_{2}$ solutions, the system was calibrated with acetone, water, and pure liquid $\mathrm{CO}_{2}$ solutions. The calibration plot and $\mathrm{C}$ values obtained are shown in Figure 2.10.

The solution viscosities of $\mathrm{CO}_{2}$-photoresist solutions determined by the falling cylinder viscometer are shown in Figure 2.11. These viscosity values were used in the calculations of the viscosity parameter described in Chapter 3. 


\section{Solubility Measurements}

Prior to developing films, it was necessary to determine the exact solubilities of the photoresist polymers. This was accomplished by conducting cloud point measurements in a high-pressure view cell [32]. For the purposes of this work, the cloud point was defined as the point below which the polymer begins to drop out of the $\mathrm{CO}_{2}$ solution, resulting in an opaque two-phase system. The most common method of determining the cloud point is visual observation [33].

To begin a measurement, the view cell was loaded with the desired amount of polymer and then filled with either liquid or supercritical $\mathrm{CO}_{2}$. The pressure in the cell was then increased such that all of the polymer dissolved and the solution became visually transparent. In the case where solubilities in liquid $\mathrm{CO}_{2}$ were required, the pressure could not be independently increased to increase solubility. Instead, the temperature of the fluid was reduced until the polymer became completely soluble. In this case, the cloud point was determined by then slowly increasing temperature until the polymer dropped out of solution. In the more traditional experiment using supercritical $\mathrm{CO}_{2}$, the view cell was connected to a variable volume hand pump (High Pressure). The pump piston was then slowly retracted, lowering the pressure, until the cloud point was observed. Data obtained using these methods were presented earlier in Tables 2.2 and 2.3.

\section{Defects in Spin Coating}

There are a variety of defects which commonly occur in conventional spin coating. Radial striations, cloudiness, and pitted surfaces (orange peel) are commonly encountered. The causes of many of these defects are poorly understood. They have 
been found to have a large dependence upon solution viscosity and solvent volatility, with several of the defects being caused by excessively rapid evaporation of the spin coating solvent. However, none of the common spin coating defects have been observed on $\mathrm{CO}_{2}$ spun films, with the exception of orange peel. Pitted surfaces are frequently generated due to particles on the surface or impurities in the photoresist solution.

One defect that plagued early $\mathrm{CO}_{2}$ spin coating efforts was the formation of large spiral waves on the wafer surfaces. These spiral waves emanated from the center of the wafer, and spiraled outward in the direction opposite rotation. Such formations are not reported in conventional spin coating literature, and were not easily identified. However, an analog to these waves was found in a paper written by Espig et al. in the 1960s describing the formation of spiral waves on a large rotating disk covered with water [34]. He concluded that the waves are the result of unstable flow which only occurs above a critical Reynolds number, i.e., $\operatorname{Re}_{\mathrm{c}}>\mathrm{A} \phi^{1 / 8}$, where $\phi$ is a parameter relating surface tension forces to viscous forces,

$$
\phi=\frac{\sigma^{3} \rho}{\mu^{4} g}
$$

Here $\sigma$ is the surface tension of the fluid, $\rho$ is the fluid density, $\mu$ is the solution viscosity, and $a$ is the angular acceleration,

$$
a=\frac{1}{2} D \omega^{2}
$$

The Reynolds number in this situation is defined as 


$$
\operatorname{Re}=\frac{\rho D}{\mu}\left|\frac{d h}{d t}\right| .
$$

Equation 2.7 does not use a standard Reynolds number velocity, but instead, uses an expression for the change in film height due to centrifugal flow, $\mathrm{dh} / \mathrm{dt}$, which is related to fluid velocity. Using the approximate formula for the film thickness as a function of time given in equation 1.1, it is possible to estimate the time rate of change of the film thickness as,

$$
\left|\frac{d h}{d t}\right|_{t=0}=2 K h_{o}^{3} ; K=\frac{\rho \omega^{2}}{3 \mu} .
$$

Using this expression for the Reynolds number, it was possible to plot the Reynolds number for our $\mathrm{CO}_{2}$ spun films vs the parameter $\phi$. As can be seen in Figure 2.12, 10\% of PMMA in a conventional spin coating solvent was securely in the stable regime. However, our $10 \%$ poly(FOMA-r-TBM)in $\mathrm{CO}_{2}$ had such a low viscosity that it was far above the critical Reynolds number, resulting in our unstable flow. The plot also explains why $20 \mathrm{wt} \%$ solutions in $\mathrm{CO}_{2}$ were far more uniform and did not exhibit this phenomenon to any large degree. Further refinements in the spin coating process increased the evaporation rate and eliminated this problem entirely.

\section{References}

1. McHugh, M.A. and V.J. Kurkonis, Supercritical Fluid Extraction: Principle and Practice. 2nd ed. 1994, Stoneham, MA: Butterworths. 
2. Cooper, A.I., Polymer synthesis and processing using supercritical carbon dioxide. Journal of Materials Chemistry, 2000. 10(2): p. 207.

3. DeSimone, J.M., et al., Cleaning process using carbon dioxide as a solvent and employing molecularly engineered surfactants. 1999, The University of North Carolina at Chapel Hill: USA.

4. DeSimone, J.M., Z. Guan, and C.S. Eisbernd, Synthesis of Fluoropolymers in Supercritical Carbon Dioxide. Science, 1992. 257: p. 945-947.

5. Romack, T.J., J.R. Combes, and J.M. DeSimone, Free-Radical Telomerization of Tetrafluoroethylene in Supercritical Carbon Dioxide. Macromolecules, 1995. 28(5): p. 1724-6.

6. DuPont, DuPont Introduces Fluoropolymers Made with Supercritical CO2 Technology. March 22, 2002.

7. Span, R. and W. Wagner, A new equation of state for carbon dioxide covering the fluid region from the triple-point temperature to $1100 \mathrm{~K}$ at pressures up to 800 MPa, in J. Phys. Chem. Ref. Data. 1996. p. 1509-1596.

8. Toriumi, M., I. Satou, and T. Itani, Vacuum ultraviolet spectra of flurocompounds for 157 nm lithography. J. Vac. Sci. Technol. B, 2000. 18(6): p. 3328-3331.

9. Kunz, R.R., et al., Outlook for 157 nm Resist Design. J. Vac. Sci. Technol. B, 1999. 17(6): p. 3267-3272.

10. Domke, W.-D., et al., Pattern Collapse in High-Aspect-Ratio DUV and 193-nm Resists. Proceedings of the SPIE Part 1, Advances in Resist Technology and Processing XVII, 2000: p. 313-321. 
11. Namatsu, H., K. Yamazaki, and K. Kurihara, Supercritical resist dryer. J. Vac. Sci. Technol. B, 2000. 18(2): p. 780-784.

12. Rindfleisch, F., T.P. DiNoia, and M.A. McHugh, Solubility of Polymer and Copolymers in Supercritical $\mathrm{CO}_{2}$. J. Phys. Chem., 1996. 100: p. 15581-15587.

13. McHugh, M.A. and V.J. Kurkonis, Supercritical Fluid Extraction: Principle and Practice. 2nd ed. 1986, Stoneham, MA: Butterworths.

14. Diep, P., et al., $\mathrm{CO}_{2}$-Fluorocarbon and $\mathrm{CO}_{2}$-Hydrocarbon Interactions from First-Principles Calculations. J. Phys. Chem. A, 1998. 102: p. 2231-2236.

15. Hoefling, T.A., R.M. Enick, and E.J. Beckman, J. Phys. Chem., 1991. 95: p. 7127.

16. Goel, S.K. and E.J. Beckman, Generation of Microcellular Polymeric Foams Using Supercritical Carbon Dioxide. Polmyer Engineering and Science, 1994. 34(14).

17. McHugh, M.A. and V.J. Krukonis, Supercritical Fluid Extraction : Principles and Practice. Second ed, ed. H. Brenner. 1994, Boston: Butterworth-Heinemann.

18. McClain, J.B., et al., Design of Nonionic surfactants for Supercritical Carbon Dioxide. Science, 1996. 274: p. 20492052.

19. Desimone, J.M., et al., Dispersion Polymerizations in Supercritical Carbon Dioxide. Science, 1994. 265: p. 356-359.

20. McClain, J.B., et al., Solution Properties of a $\mathrm{CO}_{2}$-Soluble Fluoropolymer via Small Angle Neutron Scattering. J. Am. Chem. Soc., 1996. 118: p. 917-918.

21. Ober, C.K., et al., Imaging Polymers with Supercritical Carbon Dioxide. Advanced Materials, 1997. 9(13): p. 1039-1043. 
22. DeSimone, J.M., et al., Molecularly Engineered Surfactants for CO2. Polymer Preprints, 1999. 40(1): p. 435-436.

23. Kiran, E. and Y.L. Sen, Viscosity of Polymer Solutions in Near-Critical and Supercritical Fluids. Polystyrene and n-butane. ACS Symposium Ser. (Supercritical Fluid Engineering and Science), 1993. 514: p. 104-20.

24. Raghavan, S.R. and S.A. Khan, Shear-Induced Microstructural Changes in Flocculated Suspensions of Fume Silica. Journal of Theology, 1997. 39(6): p. 1311-1325.

25. Mattischek, H.P. and R. Sobczak, A New Cell for Measurement of Viscosity Under High Pressure. Meas. Sci. Technol., 1994. 5: p. 782-785.

26. King, H.E., E. Herbolzheimer, and R.L. Cook, The Diamond-Anvil Cell as a High Pressure Viscometer. Journal of Applied Physics, 1992. 71(5): p. 2071-2081.

27. Hersey, M.D. and H. Shore, Viscosity of Lubricants Under Pressure. Mechanical Engineering, 1928. 50(3): p. 221-232.

28. Bird, R.B., R.C. Armstrong, and O. Hassager, Dynamics of Polymer Liquids, Vol 1: Fluid Mechanics. 1997, New York: Wiley.

29. Hatschek, E., The Viscosity of Liquids. 1928, New York: D. Van Nortrand Company, Inc.

30. Bae, Y.C. and E. Gulari, Viscosity Reduction of Polymeric Liquid by Dissolved Carbon Dioxide. Journal of Applied Polymer Science, 1997. 63: p. 459-466.

31. Bird, R.B., W.E. Stewart, and E.N. Lightfoot, Transport Phenomena. 1960: John Wiley \& Sons. 502. 
32. Henon, F., Environmentally Benign Polymer Coating Process for the Protection of Monumental Civil Infrastructures, in Chemical Engineering. 1999, North Carolina State Univ.: Raleigh. p. 54.

33. Wetmore, P.M., Supercritical Fluid Technology for Photoresist Developing, in Chemical Engineering. 1997, University of Massachusetts: Lowell.

34. Espig, H. and R. Hoyle, Waves in a thin liquid layer on a rotating disk. J. Fluid. Mech., 1964. 22(4): p. 671. 


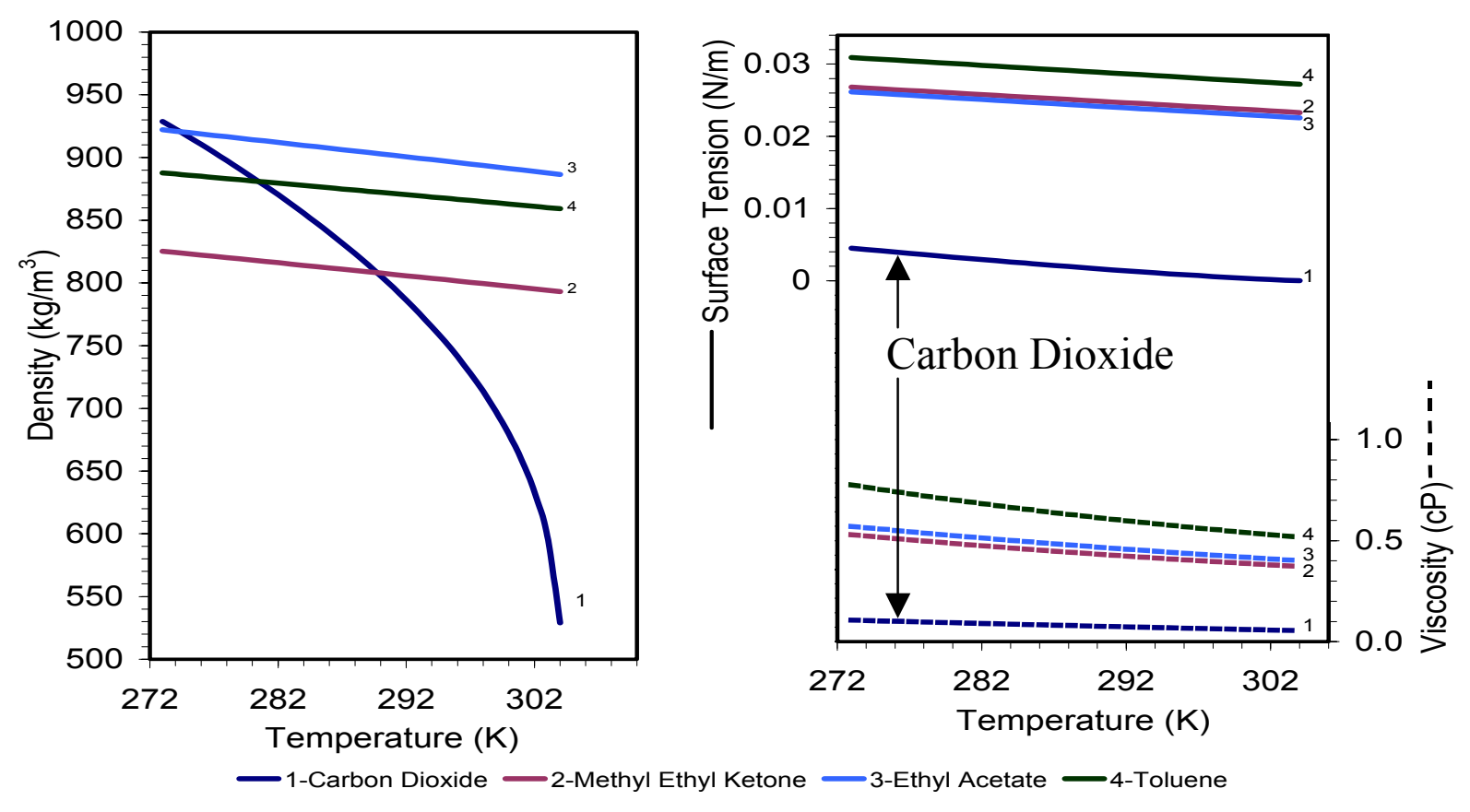

Figure 2.1. Density, surface tension and viscosity of liquid $\mathrm{CO}_{2}$ vs temperature [7]. 


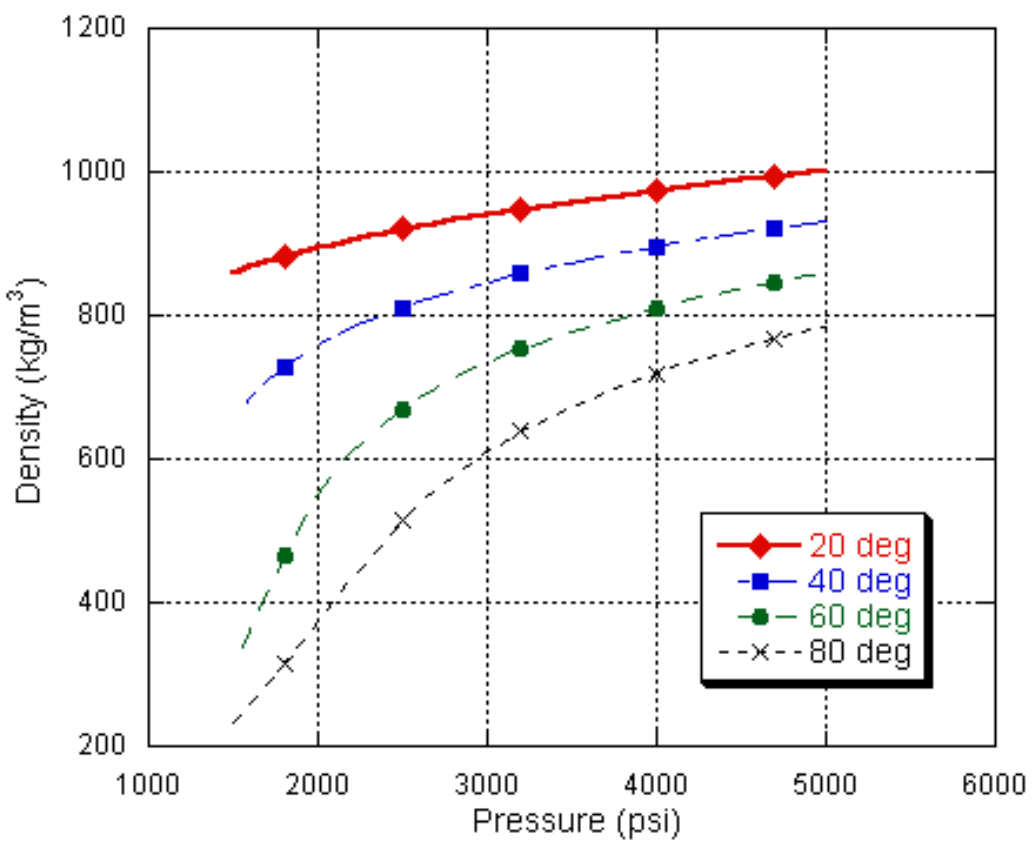

Figure 2.2. Density of dense phase $\mathrm{CO}_{2}$ vs pressure [7]. 

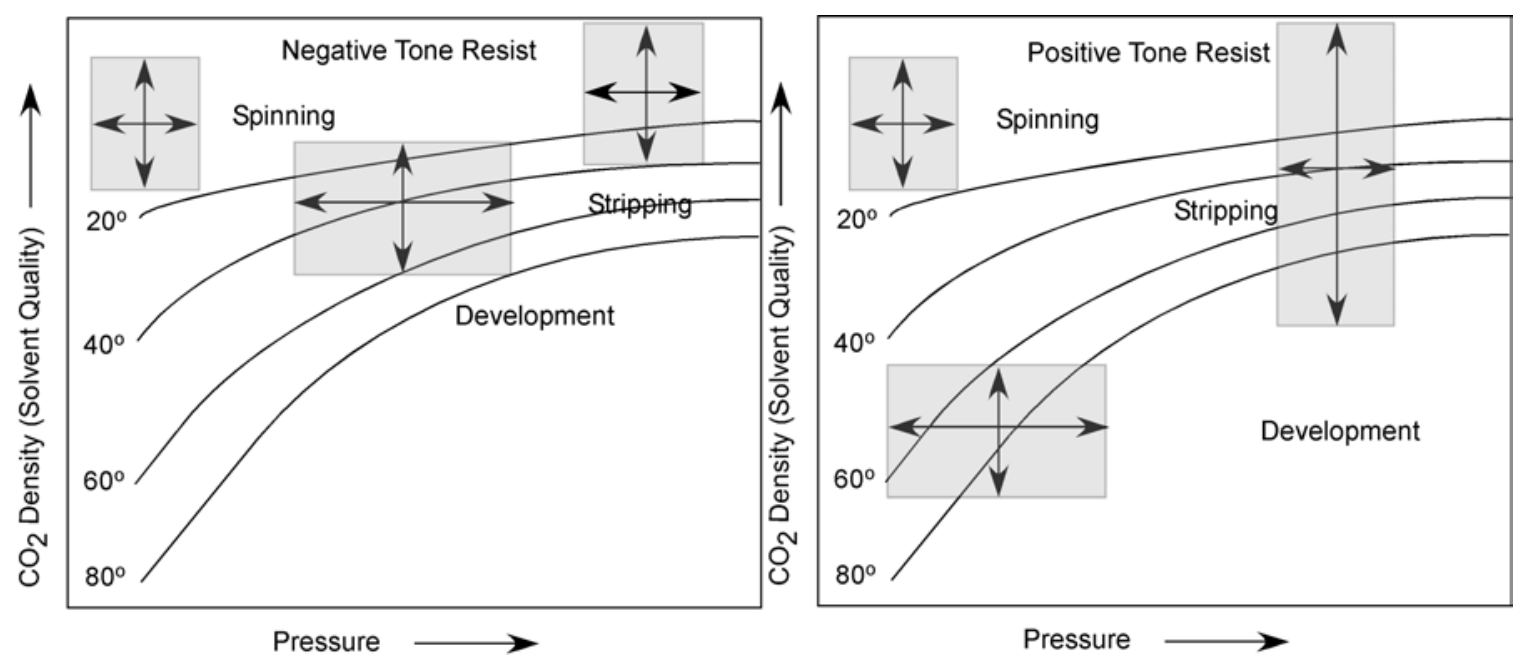

Figure 2.3. Schematic of how $\mathrm{CO}_{2}$ may be used for spinning, developing and stripping by altering the solvent strength for each step. Solvent strength is approximated here by $\mathrm{CO}_{2}$ density. 


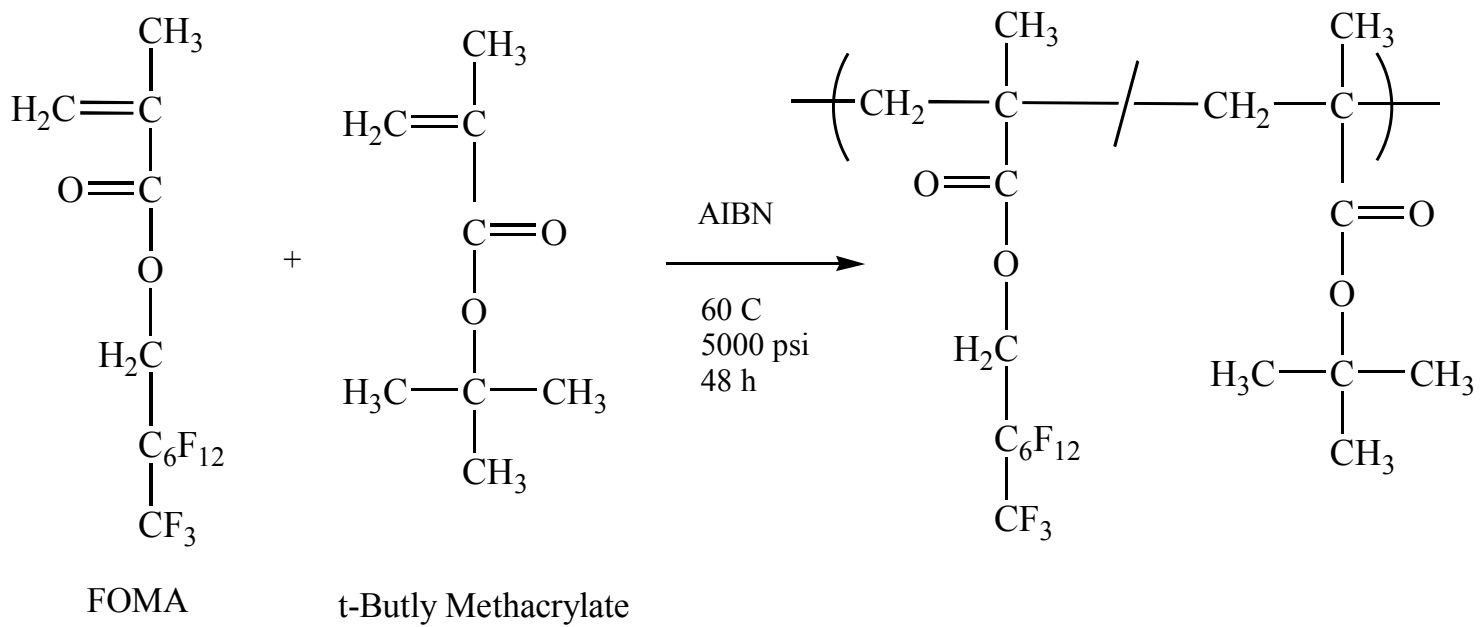

Figure 2.4. Structure of negative resist copolymer 
a)

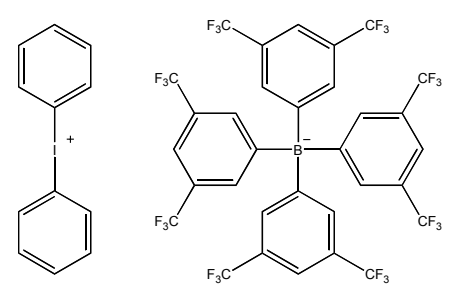

b)

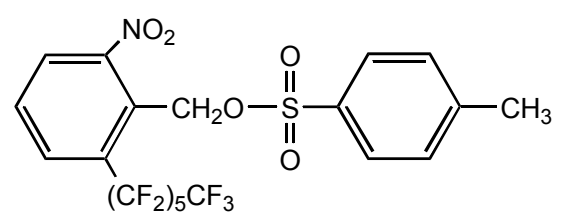

c)

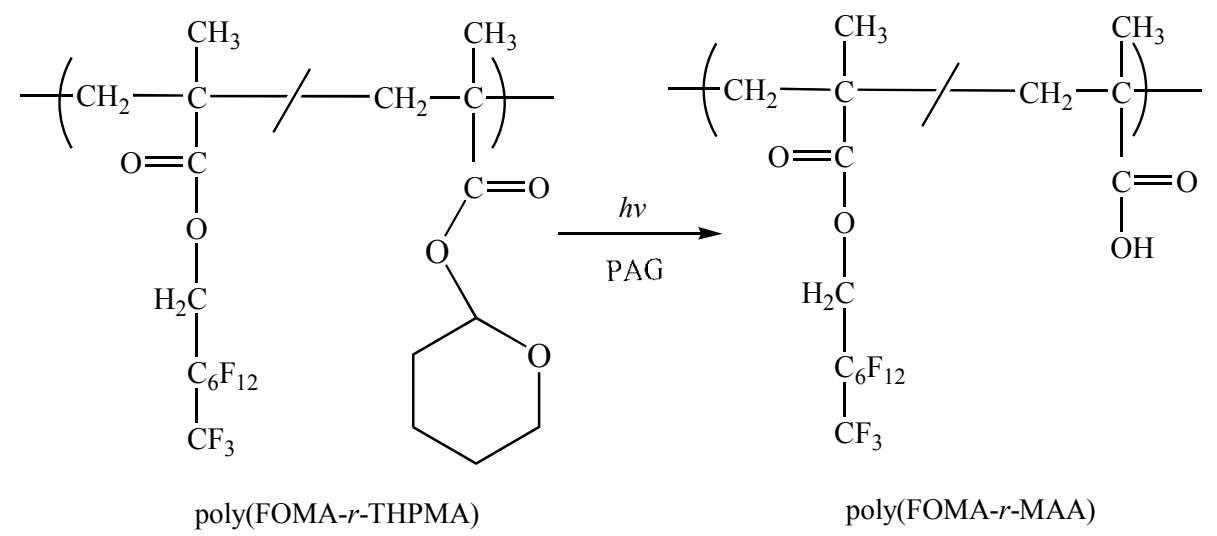

Figure 2.5. Structure of $\mathrm{CO}_{2}$ soluble photoresist system: a) ionic PAG, Diphenyliodonium tert-(3,5-bis(trifluoromethyl) phenyl boronium, b) nonionic PAG, 2perfluorohexyl-6-nitrobenzyl tosylate, c) $\mathrm{CO}_{2}$ soluble resin, poly(1,1dihydroperfluorooctyl)methacrylate 2-tetrahyrdopyranyl methacrylate. 


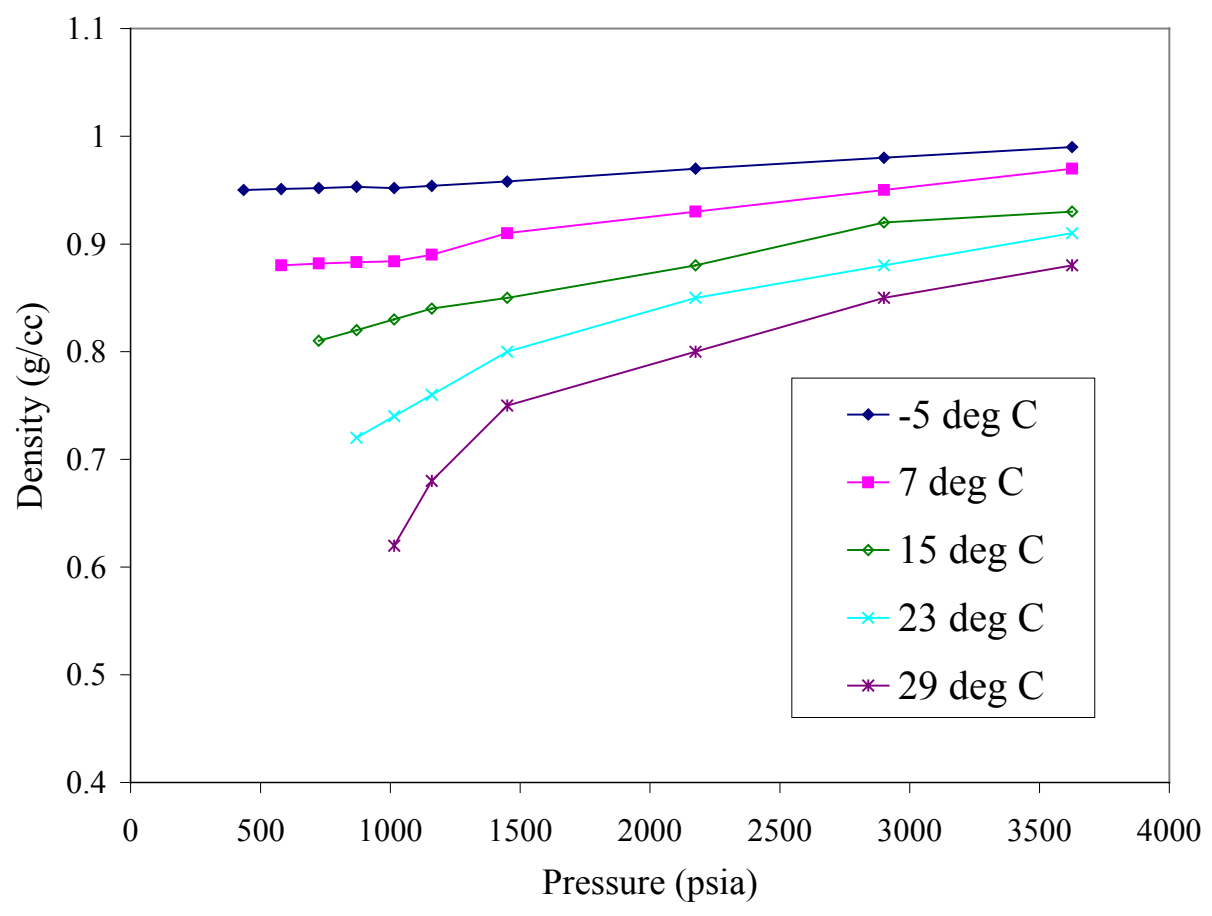

Figure 2.6. Liquid $\mathrm{CO}_{2}$ density vs pressure at various temperatures. The leftmost points on the isotherms are at the respective vapor pressures [7]. 


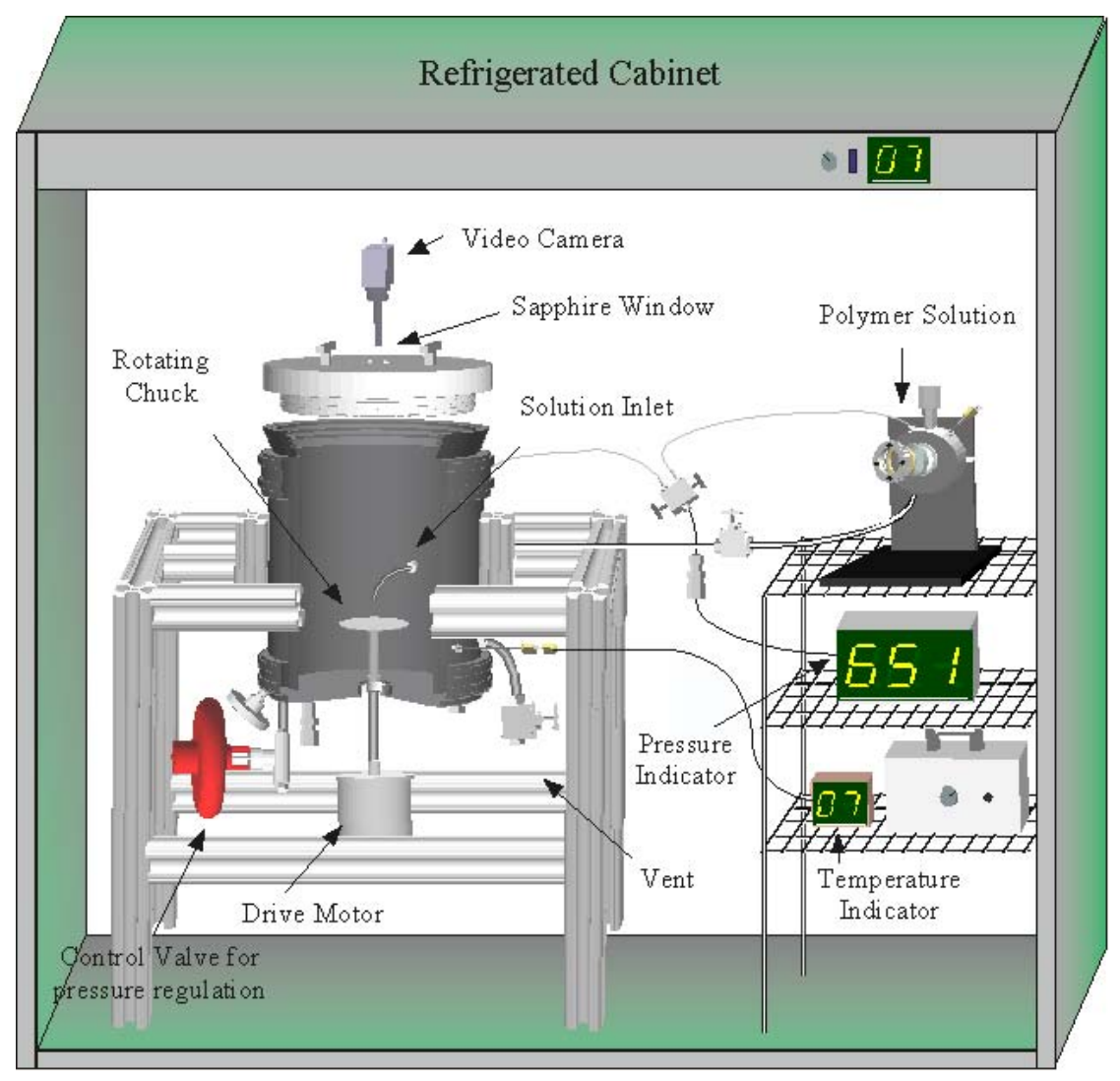

Figure 2.7a. Overview of liquid $\mathrm{CO}_{2}$ spin coating apparatus. 


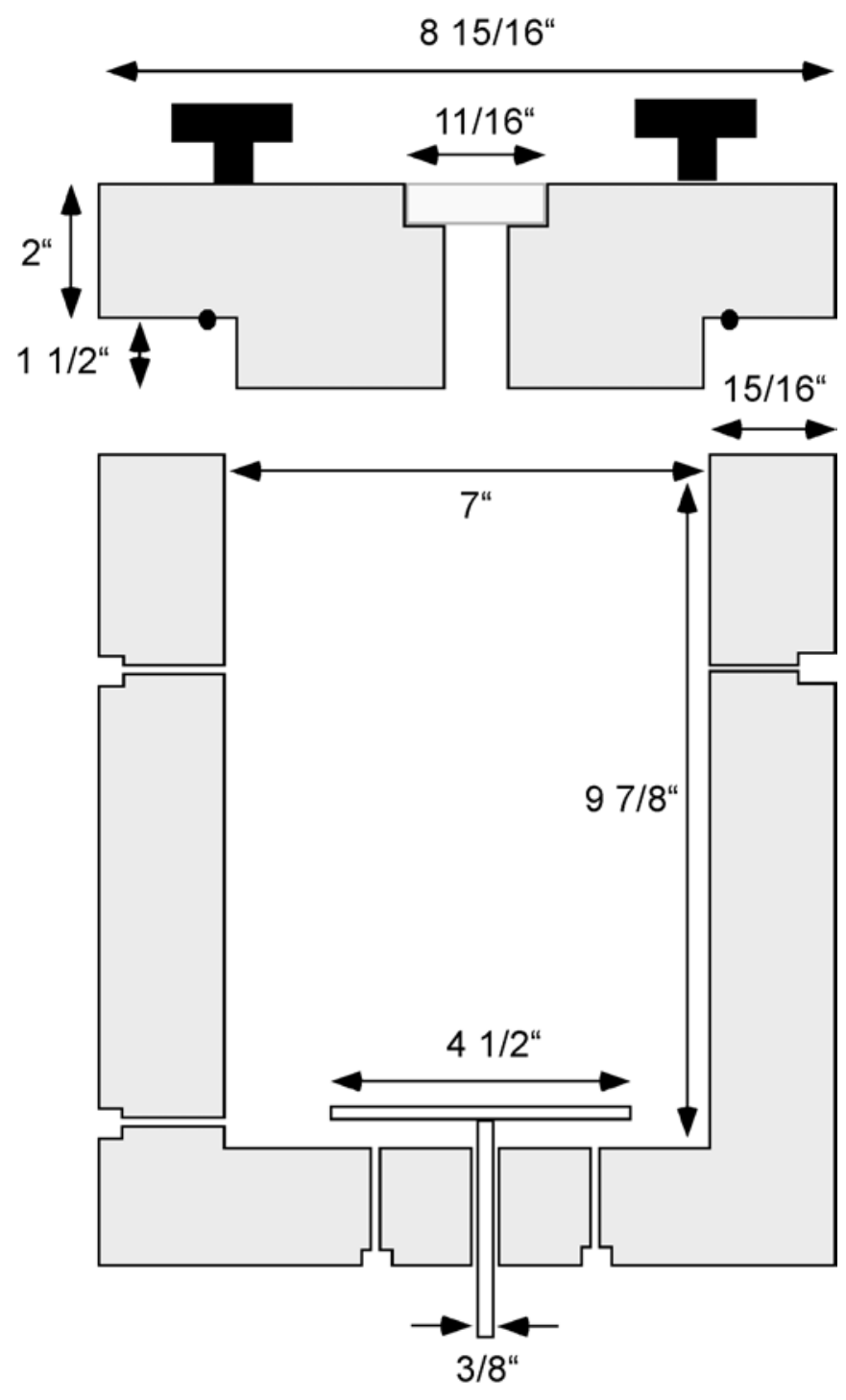

Figure 2.7b. Spin coating pressure chamber. Chamber is a modified vessel from J.D. Clipper. Total chamber volume is 6.2 litres. 


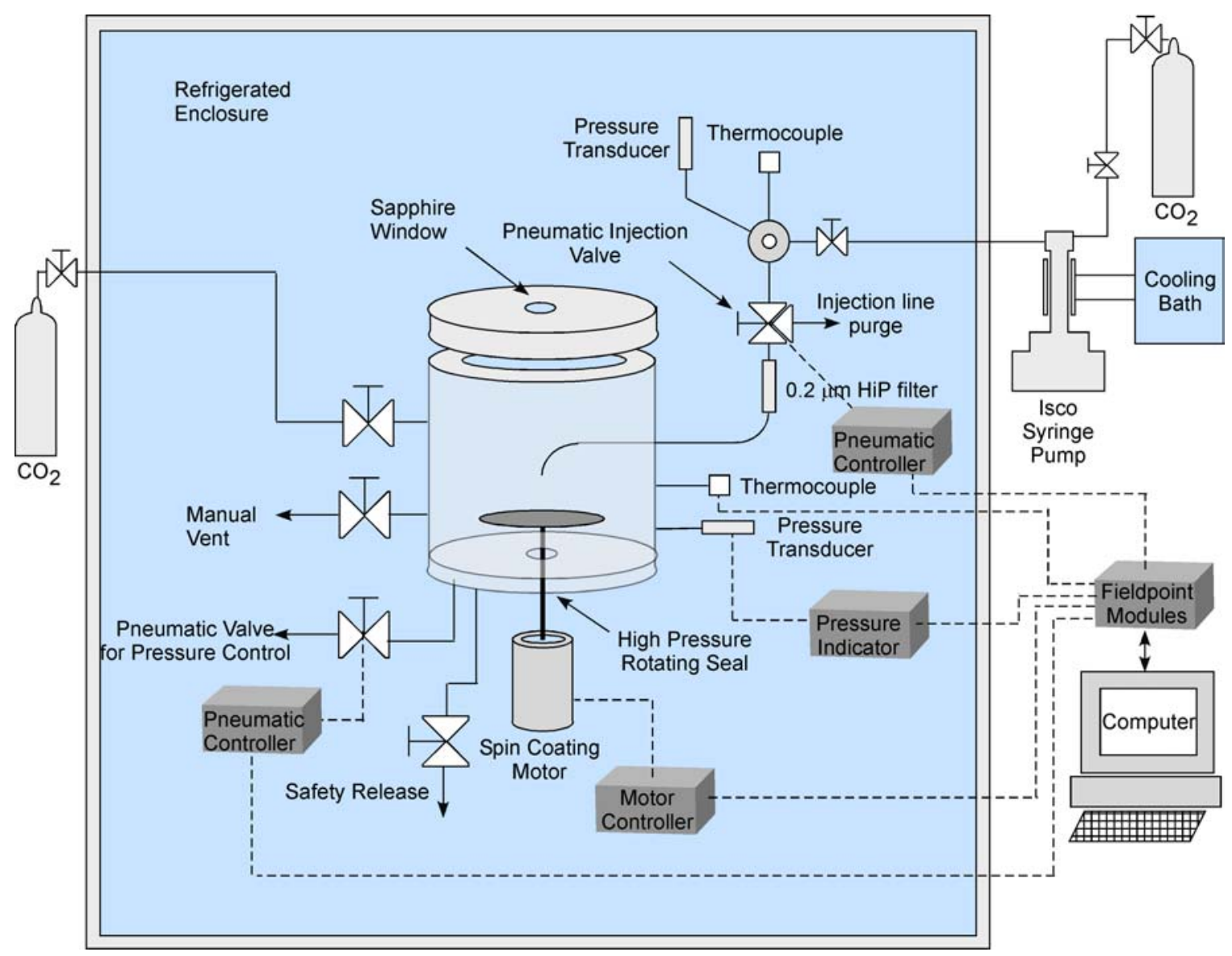

Figure 2.7c. Key components of the spin coating apparatus. High pressure tubing was a combination of $1 / 4$ ", 1/8" and 1/16" tubing (HiP). Manual valves (HiP), on/off pneumatic valves (HiP), control pneumatic valves (Badger Meter), and solenoids (McMaster) were used. Computer control was programmed with Bridgeview, and I/O between the computer, input and control devices was accomplished via Fieldpoint modules (National Instruments). 


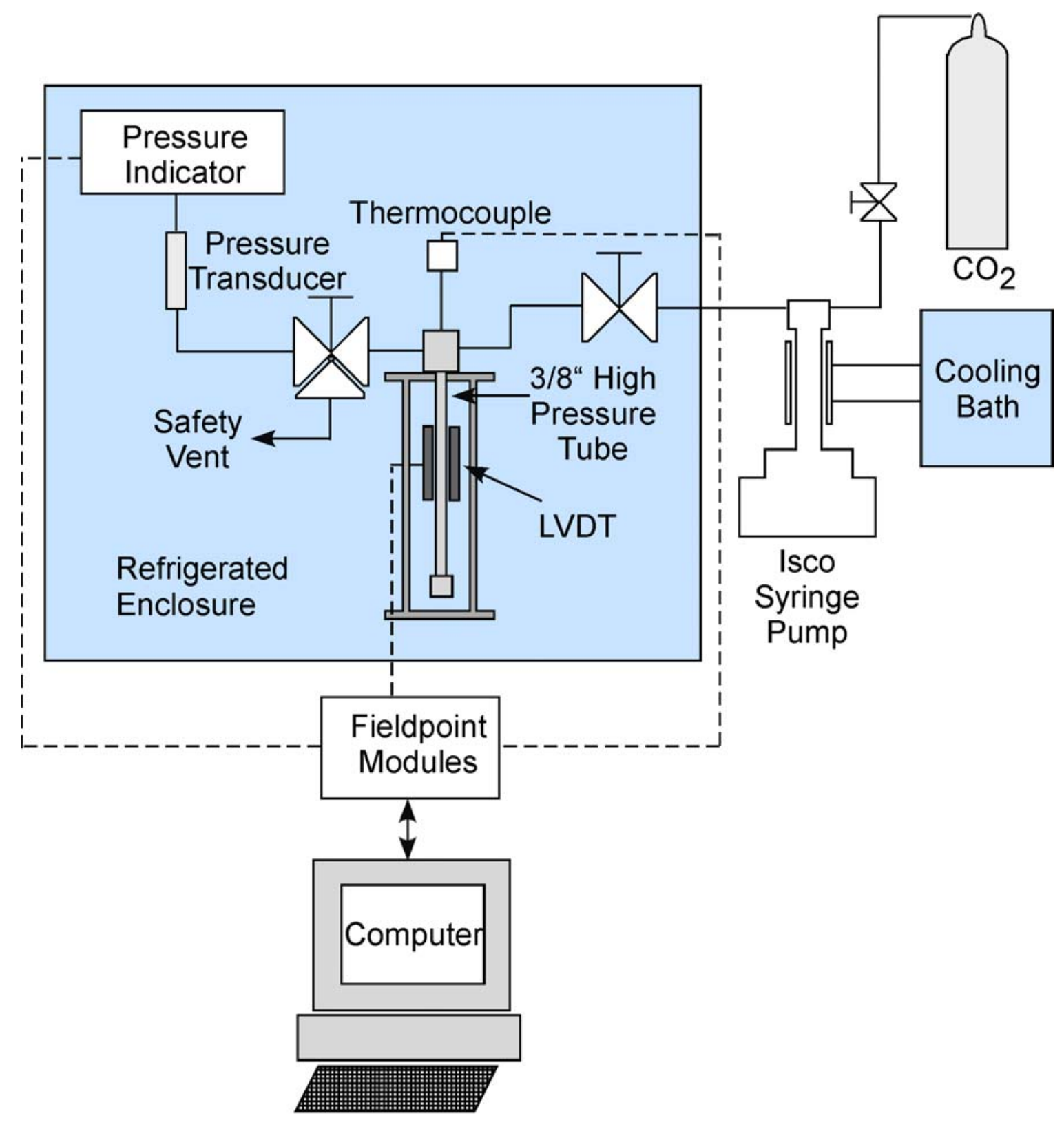

Figure 2.8. Schematic of the falling cylinder viscometer 


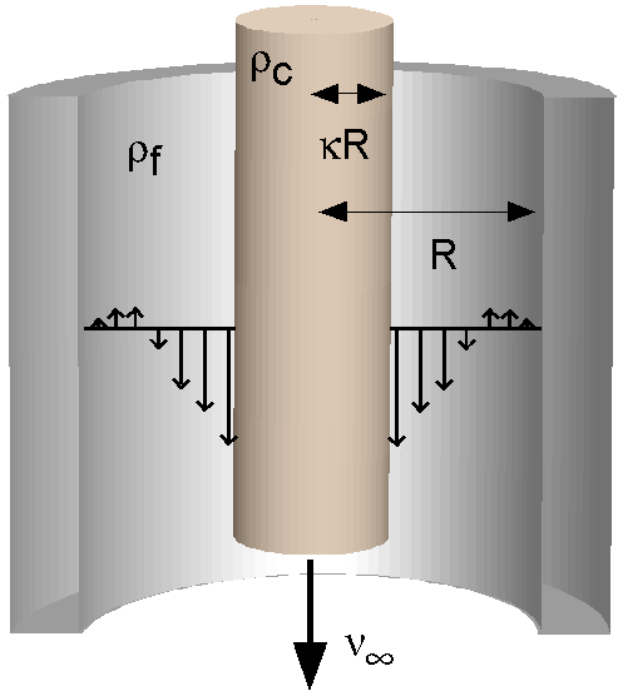

Figure 2.9. Concept of a falling cylinder viscometer 


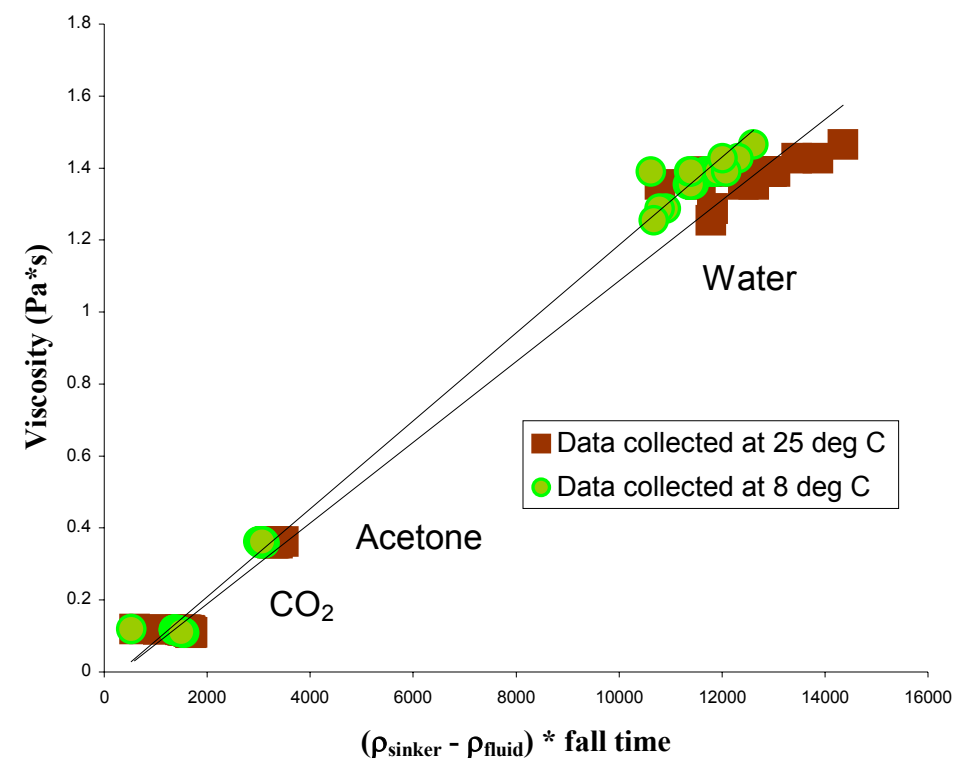

Figure 2.10. Calibration plot for the falling cylinder viscometer. Calibration constants were obtained from the slope of each line. For $25^{\circ} \mathrm{C}$, the calibration constant $\mathrm{C}$ was $1.22 \times 10^{-4}\left(\mathrm{~m}^{2} / \mathrm{s}^{2}\right)$, for $25^{\circ} \mathrm{C}$, the constant was $1.12 \times 10^{-4}\left(\mathrm{~m}^{2} / \mathrm{s}^{2}\right)$. 


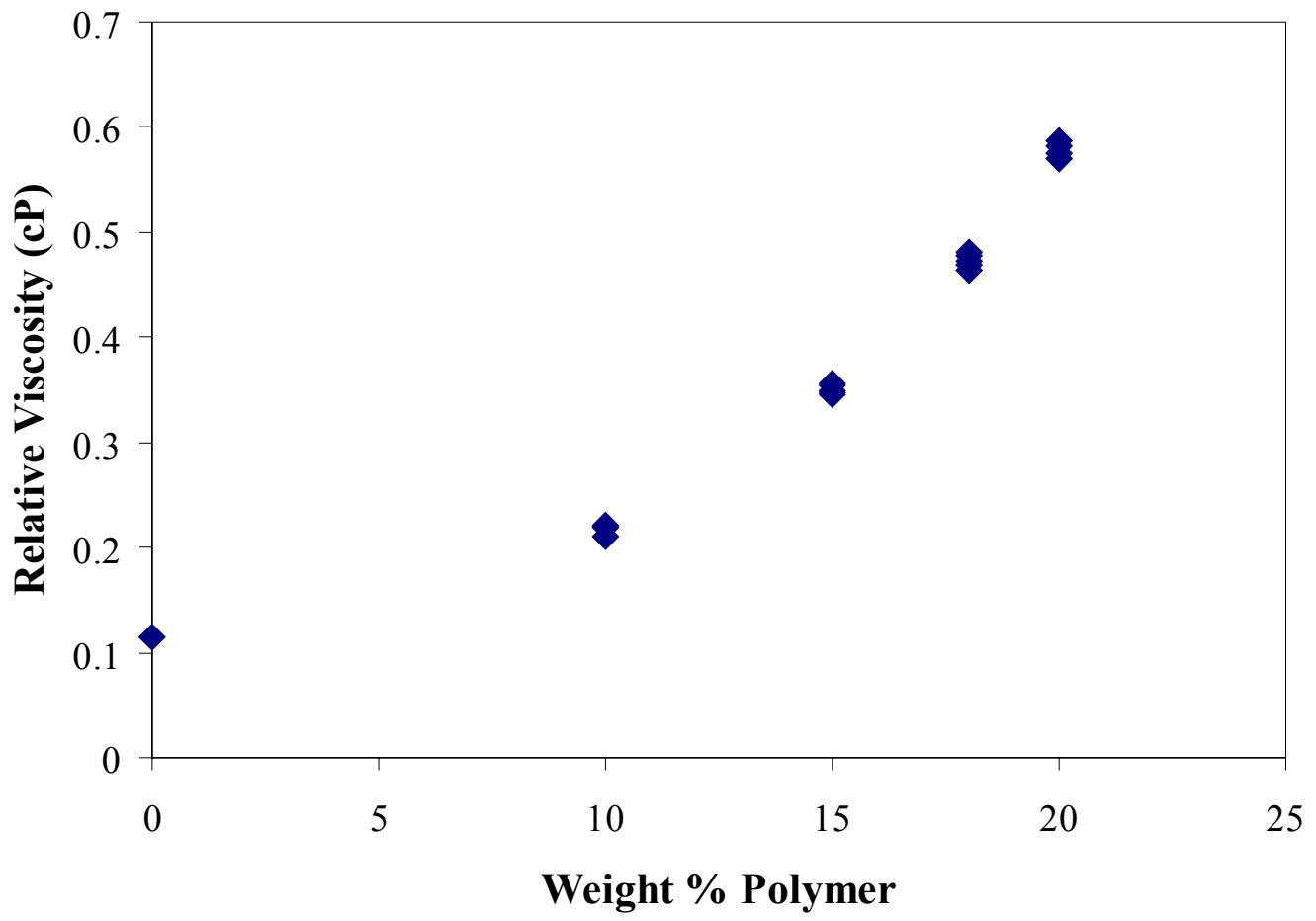

Figure 2.11. Viscosity of $\mathrm{CO}_{2} /$ poly(FOMA-r-TBM)solutions measured with the falling cylinder viscometer. 


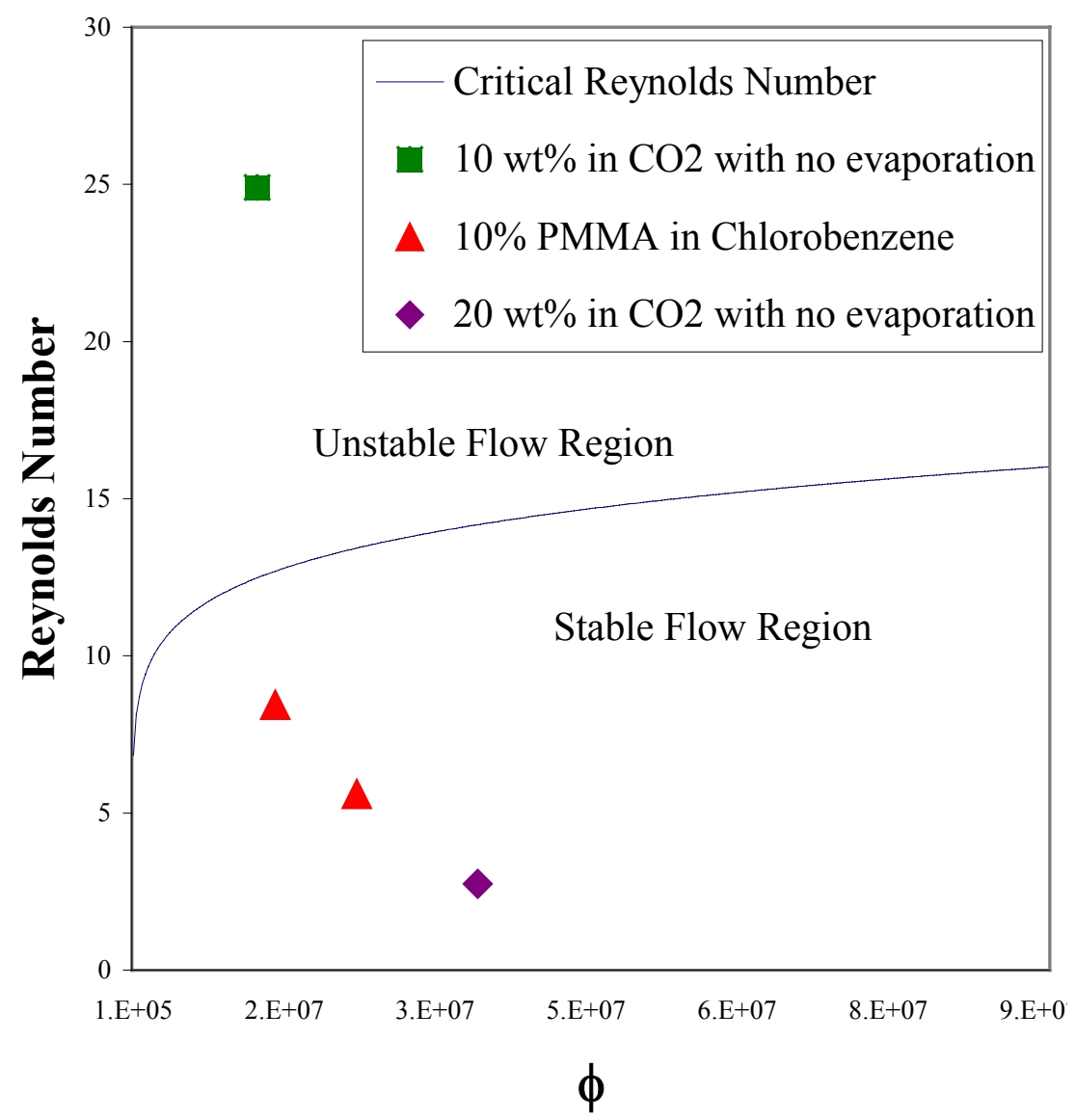

Figure 2.12. Plot of Reynolds number vs. $\phi$, demonstrating the stability limit of spun films. 


\section{Chapter 3. $\mathrm{CO}_{2}$ spin coating apparatus, theory, and coating results.}

This chapter describes the use of the high-pressure $\mathrm{CO}_{2}$ spin coating apparatus to form thin films. Included in this discussion is the development of a theoretical model for $\mathrm{CO}_{2}$ based spin coating and a comparison of that model with empirical results for film thickness and uniformity. Although patternable photoresists were used in this spin coating, their photolithographic qualities are not evaluated in this chapter, but are covered in detail in Chapters 4 and 5.

\section{Materials and Methods}

Before casting films from $\mathrm{CO}_{2}$, it was necessary to design suitable photoresists. It had been shown previously that fluorinated methacrylates could be synthesized in $\mathrm{CO}_{2}$ using a homogeneous polymerization technique, and displayed excellent solubility properties [1],[2],[3]. Building upon this previous discovery, and in light of work done in $\mathrm{CO}_{2}$ by Allen and Ober [4],[5], the first photoresists used in this work were based upon fluorinated octyl methacrylate copolymers. $\quad(1 \mathrm{H}, 1 \mathrm{H}-$ perfluorooctyl)methacrylate (FOMA) was copolymerized with t-butyl methacrylate (TBM) using azobisisobutyronitrile (AIBN) as a free radical initiator (Figure 3.1). This reaction was carried out in a high pressure $\mathrm{CO}_{2}$ vessel at $60{ }^{\circ} \mathrm{C}$ and 5000 psi. This polymer displayed excellent solubility in $\mathrm{CO}_{2}$, completely dissolving at concentrations as high as $25 \mathrm{wt} \%$. Table 2 shows the cloud points of these copolymers. When this polymer was exposed to acid, it was found that the t-butyl structures were hydrolized to methacrylic acid units, rendering the copolymer much less soluble in $\mathrm{CO}_{2}$, suggesting that it would behave well as a negative resist. 
Table 3.2. Solubilities of poly(FOMA-r-TBM)resins used.

\begin{tabular}{|c|c|c|c|}
\hline $\begin{array}{c}\text { PFOMA-TBM } \\
\text { Sample Name }\end{array}$ & Mol\% TBM in & $\begin{array}{c}\text { Wt\% of sample } \\
\text { in solution }\end{array}$ & $\begin{array}{c}\text { Cloud Point Temperature } \\
\text { (Vapor Pressure) }\end{array}$ \\
\hline KLA1-15-1 & 33 & 10 & $16.0^{\circ} \mathrm{C}(618 \mathrm{psi})$ \\
\hline & & 20 & $14.8^{\circ} \mathrm{C}(588 \mathrm{psi})$ \\
\hline KLA1-10-1 & 20 & 10 & $16.4^{\circ} \mathrm{C}(628 \mathrm{psi})$ \\
\hline & & 20 & $17.6^{\circ} \mathrm{C}(611 \mathrm{psi})$ \\
\hline JLK1-75-1 & 12 & 10 & $16.8^{\circ} \mathrm{C}(629 \mathrm{psi})$ \\
\hline & & 20 & $16.3{ }^{\circ} \mathrm{C}(621 \mathrm{psi})$ \\
\hline JLK1-71-1 & 0 & 40 & $16.6^{\circ} \mathrm{C}(629 \mathrm{psi})$ \\
\hline & & 20 & $16 .{ }^{\circ} \mathrm{C}(615 \mathrm{psi})$ \\
\hline
\end{tabular}

The spin coating was carried out in the first generation high pressure $\mathrm{CO}_{2}$ spin coater described in Chapter 2 Wafers used were $125 \mathrm{~mm}$ silicon wafers $(<100>$ orientation, boron doped, polished, International Wafer Services. The spin coating process was initiated by first dissolving the poly(FOMA-r-TBM)resin and the appropriate photoacid generator $(\mathrm{PAG})$ in a small volume $(5-10 \mathrm{ml})$ of $\mathrm{CO}_{2}$ in a high-pressure cell with sapphire windows (Inasco). The windows were used to observe the dissolution of the polymer, with full dissolution being assumed upon transparency of the mixture. Solutions were mixed at $7{ }^{\circ} \mathrm{C}$ for at least 4 hours. The nature of spin coating is such that it requires a liquid/polymer solution evaporating into the surrounding vapor. Because of this requirement supercritical $\mathrm{CO}_{2}$ was an unsuitable medium. However, it was possible to take advantage of the tunability of $\mathrm{CO}_{2}$, wherein a small decrease in temperature acts to increase the fluid density by $40 \%$ while still staying in the liquid state. This allowed us to 
dissolve sufficient concentrations of polymer by cooling the entire apparatus, placing it in a chromatography cabinet to lower the operating temperature to $7^{\circ} \mathrm{C}$.

After the solution was fully dissolved, an external computer registered the pressure inside the small cell. $\mathrm{CO}_{2}$ vapor was then added to the large spinning chamber to a specified pressure lower than the measured vapor pressure of the solution in the small cell. Precisely maintaining this relative pressure differential is key to the $\mathrm{CO}_{2}$ spin coating process. After injection of the $\mathrm{CO}_{2}$-photoresist solution, the injection line was flushed to prevent further dripping, and the wafer was spun. Spinning speeds varied from $750 \mathrm{rpm}$ to $2500 \mathrm{rpm}$. Acceleration was fixed at $5000 \mathrm{rpm} / \mathrm{min}$, and deceleration rates were $500 \mathrm{rpm} / \mathrm{min}$. After 60 seconds of spinning, the pressure in the large chamber was slowly lowered until it reached atmospheric pressure, at which time the spun wafer was then removed and analyzed. Typically spin coating times were on the order of 10 minutes per wafer, not counting loading and unloading time. Final film thicknesses ranged from $0.250 \mathrm{~mm}$ to $10 \mathrm{~mm}$, depending upon the conditions used. Films were analyzed for thickness and uniformity using profilometry, ellipsometry, and in some cases, atomic force microscopy (AFM).

It was necessary to determine values of the $\mathrm{CO}_{2}$-photoresist solution viscosity in order to predict film thicknesses and compare empirical results with theory. Viscosity measurements utilized the high-pressure falling cylinder viscometer detailed in Chapter 2. 


\section{Results}

In order to evaluate the feasibility and performance of $\mathrm{CO}_{2}$ spin coating, photoresist films were spun onto silicon at a variety of conditions in addition to those presented above.

We found that films could be cast under a wide variety of conditions, with varying degrees of quality and uniformity. Table 3.3 shows the film thickness at a variety of operating conditions.

Table 3.3. Thickness of Poly(FOMA-r-THPMA) films at various conditions (thicknesses measured via profilometry).

\begin{tabular}{|c|c|c|c|c|c|}
\hline RPM & $\mathbf{6 2 5}$ psi & $\mathbf{6 2 0}$ psi & $\mathbf{6 1 5}$ psi & 610 psi & 605 psi \\
\hline $\mathbf{7 5 0}$ & $0.4 \mu \mathrm{m}^{\ddagger}$ & $1.2 \mu \mathrm{m}^{\star}$ & $1.2 \mu \mathrm{m}$ & $1.7 \mu \mathrm{m}^{\dagger}$ & $3.1 \mu \mathrm{m}^{\dagger}$ \\
\hline $\mathbf{1 0 0 0}$ & $0.3 \mu \mathrm{m}^{\dagger}$ & $0.8 \mu \mathrm{m}^{\dagger}$ & $1.0 \mu \mathrm{m}$ & $1.0 \mu \mathrm{m}$ & $2.5 \mu \mathrm{m}^{\dagger}$ \\
\hline $\mathbf{1 5 0 0}$ & $0.2 \mu \mathrm{m}^{\dagger}$ & $0.7 \mu \mathrm{m}^{\dagger}$ & $0.9 \mu \mathrm{m}$ & $1.1 \mu \mathrm{m}$ & $2.2 \mu \mathrm{m}^{\dagger}$ \\
\hline $\mathbf{2 0 0 0}$ & $0.12 \mu \mathrm{m}^{\dagger}$ & $0.5 \mu \mathrm{m}^{\dagger}$ & $0.6 \mu \mathrm{m}$ & $0.9 \mu \mathrm{m}$ & $1.6 \mu \mathrm{m}^{\dagger}$ \\
\hline
\end{tabular}

$\ddagger \rightarrow$ Spiral waves present on film. Instability due to low viscosity.

$\dagger \rightarrow$ Defects near center of film. Evaporation rates too fast. Film partially solidifies prior to complete spin out.

As mentioned earlier, films spun with a nominal $\Delta \mathrm{P}$ of zero, i.e. no evaporation, were difficult to control precisely, but resulted in very thin films. As would be expected, the higher the evaporation rate, the thicker the final films. However, there is a clear window of optimum processing pressure where the films are of much higher quality. Photoresist films cast with a $\Delta \mathrm{P}$ of $20 \mathrm{psi}$ or greater resulted in very rapid evaporation and nonuniform polymer distribution over the wafer. Such films were unsuitable for further 
lithographic studies. Films cast with $\Delta$ Ps ranging from 10 to 15 psi produced the best results.

Not surprisingly, higher concentration (higher viscosity) solutions produced thicker films. It was found that very low concentration solutions did not produce as high quality films due to instabilities introduced into the system because of very high Reynolds numbers, in Chapter 2. Solution concentrations beyond $25 \mathrm{wt} \%$ were not investigated due to the solubility limit of the material in $\mathrm{CO}_{2}$. As expected, we found that higher rotational speeds resulted in thinner films. Lower quality films were obtained at lower speeds, but this may have been due to increased wobble in the chuck rotation at lower RPMs. Rotational speeds beyond 2500 RPMs were not investigated due to limitations in the design of the system. Values for solution viscosity were obtained with the falling cylinder viscometer mentioned above. Viscosities for solutions up to $20 \mathrm{wt} \%$ polymer are shown in Figure 3.3.

When examined by AFM, films spun under optimum conditions were found to be quite smooth (r.m.s. roughness of $0.4-0.5 \mathrm{~nm}$ ) and comparable in quality to films spun from organic solvents (Figure 3.2).

\section{Theory}

The first detailed mathematical analysis of spin coating was conducted by Emslie, Bonner, and Peck in the 1950s [6]. In their analysis, they neglected the effect of evaporation and the resultant viscosity and concentration gradients. Meyerhofer expanded upon their analysis and those results have been extended even more recently by Bornside et al. and Yonkoski et al. [7],[8],[9]. 
The conclusion reached by many of the above authors is that the spin coating process can be generally considered in four phases. In the first stage the liquid solution is deposited on the substrate, covering the entire surface. In the second stage, spin-up, the rotational speed of the substrate is rapidly increased to its final value. Acceleration rates of $10,000 \mathrm{rpm} / \mathrm{s}$ are common in the spin-up stage. Ideally, very little film thinning occurs in this step. The two final steps, spin-off and evaporation, occur concurrently. Final rotation speeds of 5,000 to $10,000 \mathrm{rpm}$ are common in conventional spin coating, although slower speeds were used in this work. At the beginning of the spin-off step, the film is several microns thick and the fluid lost to centrifugal force is far greater than that lost to evaporation. However, as the film becomes thinner, relatively little solution is flung off the wafer and evaporation becomes the primary method of film thinning. The spin coating process is typically completed in less than a minute [10],[11]. The spin-up, spin-off and evaporation stages are shown in Figure 3.3. In the figure, region I would be the spin-up stage wherein no evaporation occurs, region II would be both the spin-off and evaporation stages (they occur concurrently), and region III would be the spinning after the film has reached its final thickness.

To model this process, we begin with a coupling of the averaged momentum and continuity [7]. For the polymer- $\mathrm{CO}_{2}$ solution spinning in region I,

$$
\frac{\partial H}{\partial t}=-\frac{2 \rho \Omega^{2}}{3 \mu} H^{3}-E .
$$

Here $\mathrm{H}$ is the film thickness, $\Omega$ is the rotational speed, $\rho$ is the density, $\mu$ is the viscosity, and $\mathrm{E}$ is the film loss due to evaporation, and is not to be confused with a true molar flux. 
Equation 3.1 is very similar to equation A.13, with the notable addition of the evaporative term, and the interested reader is again referred to Appendix A for details of its derivation. An analog expression to Equation A.13 may also be derived for the solute [7] and can be written in the form,

$$
\frac{\partial(\varphi H)}{\partial t}=-\varphi \frac{2 \rho \Omega^{2} H^{3}}{3 \mu(\varphi)}
$$

where $\varphi$ is the volume fraction of polymer in the solution.

As discussed in Chapter 1, for the spin-up stage (region I - Figure 3.3) of the spin coating process, where solvent flow dominates and evaporation may be neglected, the film thickness can be described by the equation [6],

$$
H=H_{i}\left[1+\frac{4 \rho \Omega^{2}}{3 \mu_{o}} H_{i}^{3} t\right]^{-1 / 2}
$$

where $\mathrm{H}_{\mathrm{i}}$ is the initial film height, and $\mu_{\mathrm{o}}$ is the initial viscosity. Again, note that equation 3.3 is the same as Equation 1.1 as derived previously. This equation yields the wet film in the case of no evaporation. However, we are not interested in the film thickness during spin up where the volume fraction of polymer does not change, but rather the film thickness after solvent evaporation. Thus we must extend beyond the convection regime into the evaporation regime(during region II). This is done by examining the transition point, at which the film thinning due to flow is equal to the film thinning due to evaporation. This point is designated $t_{o}$ in Figure 3.4. By definition, at this point the film thinning due to evaporation is equal to the rate of thinning by centrifugal forces, 


$$
E=\left(1-\varphi_{o}\right) \frac{2 \Omega^{2} \rho H_{o}^{3}}{3 \mu_{o}} .
$$

Equation 3.4 relates the film loss due to evaporation to the film loss due to the centrifugal loss of solvent. If we assume that evaporation is the dominant force for film thinning in region II, then the final dry film thickness $\mathrm{H}_{\mathrm{f}}$ must be equal to the volume fraction of polymer multiplied by the film height at the beginning of that region $\left(\mathrm{H}_{\mathrm{f}}=\phi_{\mathrm{o}} \mathrm{H}_{\mathrm{o}}\right)$. Thus Equation 3.4 may be solved for $H_{o}$ and then multiplied by $\phi_{o}$ to yield the dry film thickness,

$$
H_{f}=\varphi_{o} H_{o}=\varphi_{o}\left[\frac{3 \mu_{o} E}{2\left(1-\varphi_{o}\right) \rho \Omega^{2}}\right]^{1 / 3} .
$$

Equation 3.5 may be applied to both conventional and $\mathrm{CO}_{2}$ spin coating. The difference is in the expression for the film loss due to evaporation, E. In conventional spin coating, the evaporation rate is dependent upon the diffusion of the solvent vapor away from the surface and may be written as,

$$
E=\frac{\dot{m}}{\rho}=\frac{k_{m}\left[C^{*}-C_{\infty}\right] M}{\rho} .
$$

Here $m$ is the mass flux due to evaporation, $\mathrm{C}^{*}$ is the concentration of solvent at the surface, $\mathrm{C}_{\infty}$ is the concentration of solvent in the bulk phase, and $\mathrm{M}$ is the molar mass of the solvent. For the case of a rotating disk, it is known that [12]

$$
k_{m}=0.6205 v^{-1 / 6} D^{2 / 3} \Omega^{1 / 2} .
$$


Here $v$ is the fluid kinematic viscosity, and $\Omega$ is the rotational speed of disk. Thus, it is possible to combine Equations 3.5 and 3.6 and represent the change in film height due to evaporation as [8],

$$
E=c_{1} \Omega^{1 / 2}
$$

where $\mathrm{c}_{1}$ is a parameter containing the diffusion coefficient. However, in the case of $\mathrm{CO}_{2}$ spin coating, there is no diffusion gradient because the atmosphere inside the chamber is composed entirely of $\mathrm{CO}_{2}$ vapor. Thus the evaporation rate is dependent not upon the rotational speed, but rather upon the difference between the chamber pressure and the vapor pressure of the $\mathrm{CO}_{2}$-photoresist solution. The Hertz-Knudsen equation, based on the statistical mechanics of gases, states that the molar evaporative flux for a single component system is given by [13],

$$
\frac{d N}{A_{e} d t}=\frac{\alpha_{v}}{N_{a} \sqrt{2 m k_{B} T}} \cdot \Delta P
$$

Here $\left(1 / \mathrm{A}_{\mathrm{e}}\right) \mathrm{dN} / \mathrm{dt}$ is the molar evaporative flux, $m$ is the mass of the molecule, $\mathrm{k}_{\mathrm{B}}$ is the Boltzmann constant, $\alpha_{v}$ is a sticking coefficient, $N_{a}$ is Avogadro's number, $T$ is the temperature, and $\Delta \mathrm{P}$ is the difference between the operating pressure and the vapor pressure of the component. The evaporative flux may be related to the quantity $\mathrm{E}$ as

$$
E=\frac{d N}{d t} \cdot \frac{M}{\rho}
$$


Equations 3.8 and 3.9 may be combined and simplified by lumping the parameters into an evaporation coefficient, $\mathrm{k}$,

$$
E=k \Delta P \text {. }
$$

Thus, for the case of spin coating in $\mathrm{CO}_{2}$, the evaporative loss is not proportional to the square root of the rotational speed, but rather is proportional to the difference between the vapor pressure of the fluid and the chamber pressure. Combining equation 3.11 with equation 3.5 yields an expression for the final film thickness,

$$
H_{f}=\left(\frac{3}{2}\right)^{1 / 3} k^{1 / 3} \varphi_{o}\left(1-\varphi_{o}\right)^{-1 / 3} \mu_{o}^{1 / 3} \rho^{-1 / 3} \Omega^{-2 / 3} \Delta P^{1 / 3}
$$

Equation 3.12 yields the final dry film thickness as a function of initial solution properties including initial polymer volume fraction, viscosity, density, and two process conditions, namely rotational speed and pressure differential.

\section{Comparison of Theory and Experiment}

A variety of films were spun from $\mathrm{CO}_{2}$ and compared to the theory. All films discussed here were cast of poly(FOMA-r-TBM)copolymer with a $\mathrm{M}_{\mathrm{w}}$ of approximately 25,000. To determine the effect of each variable, processing conditions were fixed with the exception of the variable to be studied.

To determine the effect of rotational speed on film thickness, films were spun of 20 wt $\%$ copolymer dissolved in $\mathrm{CO}_{2}$ with an evaporative driving force of $10 \mathrm{psi}$, i.e., the pressure in the spinning chamber was maintained at 10 psi below the vapor pressure of 
the $\mathrm{CO}_{2}$-photoresist solution. Figure 3.4 shows the measured thicknesses of these films plotted versus $1 / \Omega^{2 / 3}$. As can be seen, the data points fall on nearly a straight line, as is predicted by equation 3.12 . These data correspond very well to the trend predicted by the theory.

An additional group of parameters investigated was that relating to solution viscosity and concentration. According to Equation 3.12, the final film height should be proportional to the viscosity and volume fraction of polymer such that

$$
H_{f} \propto \varphi_{o}\left(\frac{\mu_{o}}{\left(1-\varphi_{o}\right)}\right)^{1 / 3}
$$

To calculate values for this viscosity parameter, the volume fraction of the polymer in the initial solution was calculated using the linear average of the densities of the liquid $\mathrm{CO}_{2}\left(0.886 \mathrm{~kg} / \mathrm{m}^{3}\right)[14]$ and the pure polymer $\left(1.6 \mathrm{~kg} / \mathrm{m}^{3}\right)$ assuming no volume change upon mixing. The initial solution viscosities were obtained as discussed above. Figure 3.5 contains a plot of the final film thickness plotted versus this viscosity parameter. The data plotted in this manner are nearly linear, suggesting once again that equation 3.9 seems to provide a valid representation of the system behavior.

The final experiments performed were to evaluate the effect of the evaporative driving force, $\Delta \mathrm{P}$, on the film thickness. A series of films were spun from $20 \mathrm{wt} \%$ copolymer in $\mathrm{CO}_{2}$, at a rotational speed of 1500 RPM. Because initial solution viscosity, density, and concentration were all fixed, equation 3.12 predicts that the resultant film thicknesses from these trials should be proportional to $\Delta \mathrm{P}^{1 / 3}$. Figure 3.4 shows that this is indeed the case, showing good agreement between the experimental data and the theory. It should 
be noted that films spun with a pressure differential of zero resulted in a non-zero film thickness. Such films were found to be very thin $(<0.2 \mu \mathrm{m})$ and displayed significant nonuniformities. Equation 3.12 predicts a zero film thickness at a pressure differential of zero, resulting in an apparent contradiction between this data and the proposed theory. The reason for this discrepancy seems to be the limitations in the pressure control system for the apparatus. The apparatus was designed to precisely maintain the pressure in the chamber relative to the vapor pressure of the solution, however, there was an error of approximately $0.3 \mathrm{psi}$ in the pressure transducer used. Coupled with the noninstantaneous response of the PID controller, small deviations from $0 \Delta \mathrm{P}$ were observed.

To evaluate the effect of small variations from zero $\Delta \mathrm{P}$, equation 3.12 was applied to all of the non-zero $\Delta \mathrm{P}$ experimental data. Since all quantities in equation 3.9 were known except for the $\mathrm{k}$ values, it was possible to fit the data to determine a value for $\mathrm{k}$. A $\mathrm{k}$ value of $228.7 \mathrm{~kg} /\left(\mathrm{Pa}^{2} \cdot \mathrm{s}^{3}\right)$ was found to provide the best fit of the data. Using this value of $\mathrm{k}$ it was possible to calculate the predicted dry film thickness for very small, but nonzero $\Delta \mathrm{P}$ values. Results of these calculations are given in Table 3.4.

Table 3.4. Predicted film thicknesses for low $\Delta \mathrm{P}$.

\begin{tabular}{|c|c|}
\hline$\Delta \mathbf{P}(\mathbf{p s i})$ & Predicted Dry Film Thickness $(\mu \mathbf{m})$ \\
\hline 3.0 & 0.62 \\
\hline 2.0 & 0.54 \\
\hline 1.0 & 0.43 \\
\hline 0.5 & 0.34 \\
\hline 0.1 & 0.20 \\
\hline
\end{tabular}


As can be seen from Table 3.4, even very small deviations from zero $\Delta \mathrm{P}$ result in significant film thicknesses. Since the pressure control for the spin coater is not accurate to that precision, the significant film thicknesses at a nominal $\Delta \mathrm{P}=0$ are most likely due to this small fluctuation in pressure. It was not possible to include film thicknesses for $\Delta \mathrm{P}$ values of greater than $20 \mathrm{psi}$ due to the large film defects caused by too rapid evaporation.

\section{Conclusions}

We have successfully shown that high quality, uniform thin films of photoresist polymer may be spun using only $\mathrm{CO}_{2}$ as the solvent. Film thicknesses match up very well against a proposed theoretical model, allowing the prediction of film thickness based upon key operating parameters such as solution concentration, rotational speed, and evaporative driving force. While there remains much work to be done to improve this new process, we have shown that it is indeed possible to produce lithographic quality films using only liquid $\mathrm{CO}_{2}$ as a solvent. The replacement of conventional organic solvents in this process promises many benefits, and opens the way for the use of $\mathrm{CO}_{2}$ in all areas of photolithography in the future.

Acknowledgement. The author thanks Dario Goldfarb of IBM Corporation, Yorktown Heights, NY, for his assistance with the AFM data.References 


\section{References}

1. DeSimone, J.M., Z. Guan, and C.S. Eisbernd, Synthesis of Fluoropolymers in Supercritical Carbon Dioxide. Science, 1992. 257: p. 945-947.

2. McClain, J.B., et al., Solution Properties of a $\mathrm{CO}_{2}$-Soluble Fluoropolymer via Small Angle Neutron Scattering. J. Am. Chem. Soc., 1996. 118: p. 917-918.

3. McClain, J.B., et al., Design of Nonionic surfactants for Supercritical Carbon Dioxide. Science, 1996. 274: p. 20492052.

4. Allen, R.D. and G.M. Wallraff. 1997: U.S.

5. $\quad$ Ober, C.K., et al., Imaging Polymers with Supercritical Carbon Dioxide. Advanced Materials, 1997. 9(13): p. 1039-1043.

6. Emslie, A.G., F.T. Bonnner, and L.G. Peck, Flow of a Viscous Liquid on a Rotating Disk. J. Appl. Phys., 1957. 29(5).

7. Meyerhofer, D., Characteristics of Resist Films Produced by Spinning. J. Appl. Phys., 1978. 49(7).

8. Bornside, D.E., C.W. Macosko, and L.E. Scriven, Spin Coating: OneDimensional Model. J. Appl. Phys., 1989. 66(11): p. 5185-5193.

9. Yonkoski, R.K. and D.S. Soane, Model for Spin Coating in Microelectronic Applications. J. Appl. Phys., 1992. 72(2).

10. Birnie, D.P., Combined Flow and Evaporation During Spin Coating of Complex Solutions. Journal of Non-Crystalline Solids, 1997. 2118: p. 174-178. 
11. Paul, S., et al., An Anthracene-Containing PMMA Derivative for Photoresist and Channel Waveguide Applications. Thin Solid Films, 1996. 288: p. 150-154.

12. Geankoplis, C.J., Transport Process and Unit Operations. Third ed. 1993: Simon \& Schuster. a)464, b)462, c)463, d)395.

13. L.V. Gurvich, I.V.V., C. B. Alcock, Thermodymanic Properties of Individual Substances, Fourth Edition. 1989, New York: Hemisphere Publishing Corporation.

14. Span, R. and W. Wagner, A new equation of state for carbon dioxide covering the fluid region from the triple-point temperature to $1100 \mathrm{~K}$ at pressures up to 800 MPa, in J. Phys. Chem. Ref. Data. 1996. p. 1509-1596. 


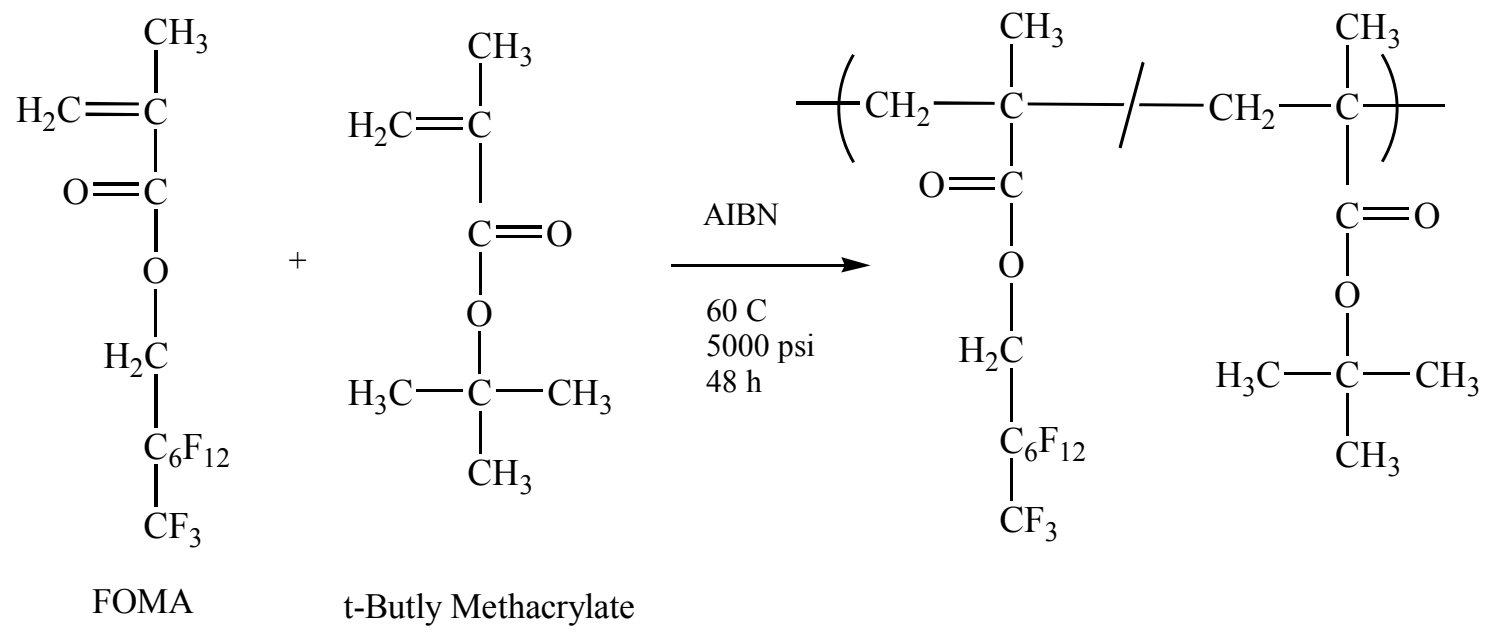

Figure 3.1. Synthesis of poly(FOMA-r-TBM)in supercritical $\mathrm{CO}_{2}$. 


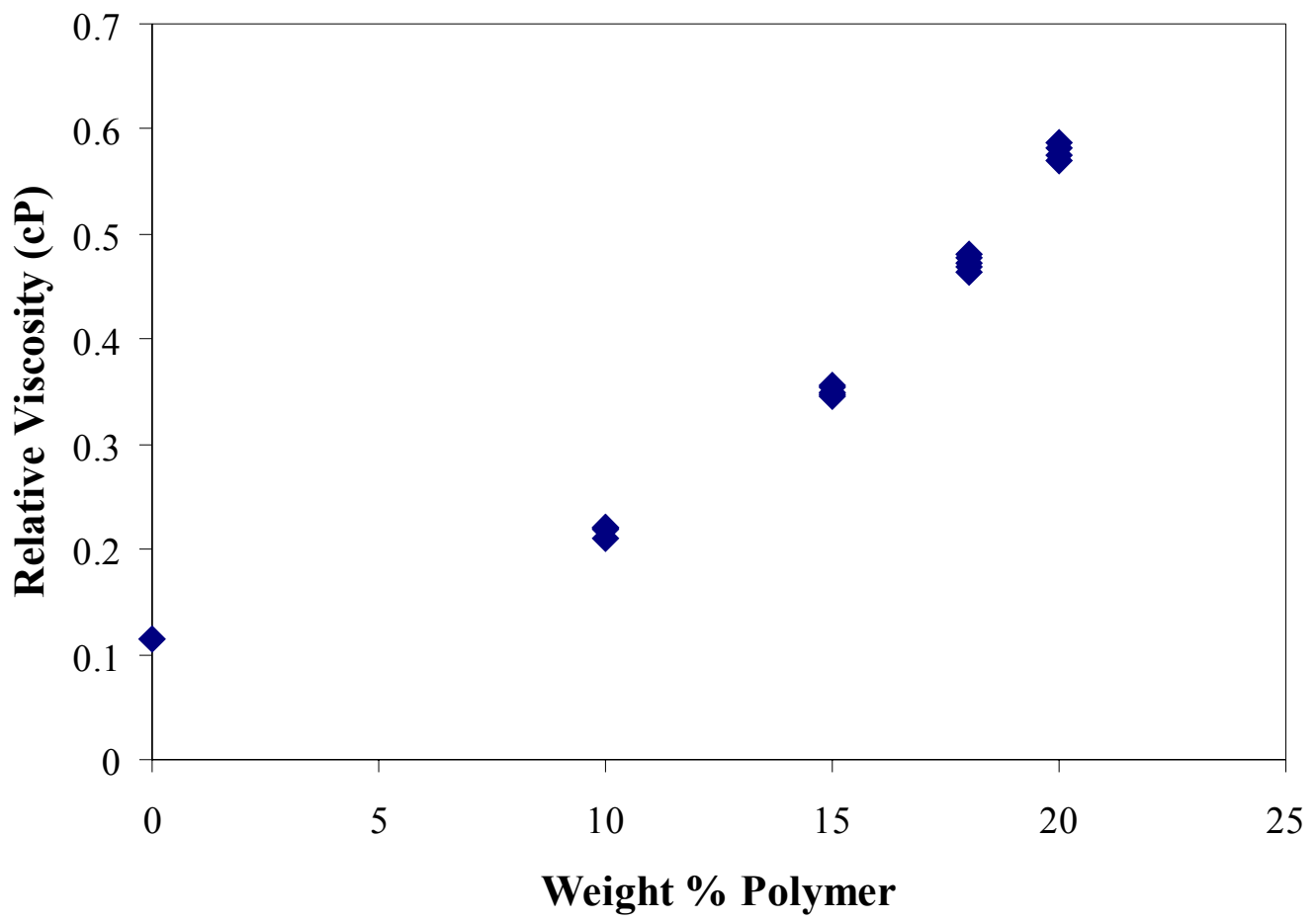

Figure 3.2. Viscosity of $\mathrm{CO}_{2} /$ poly(FOMA-r-TBM)solutions measured with the falling cylinder viscometer. 


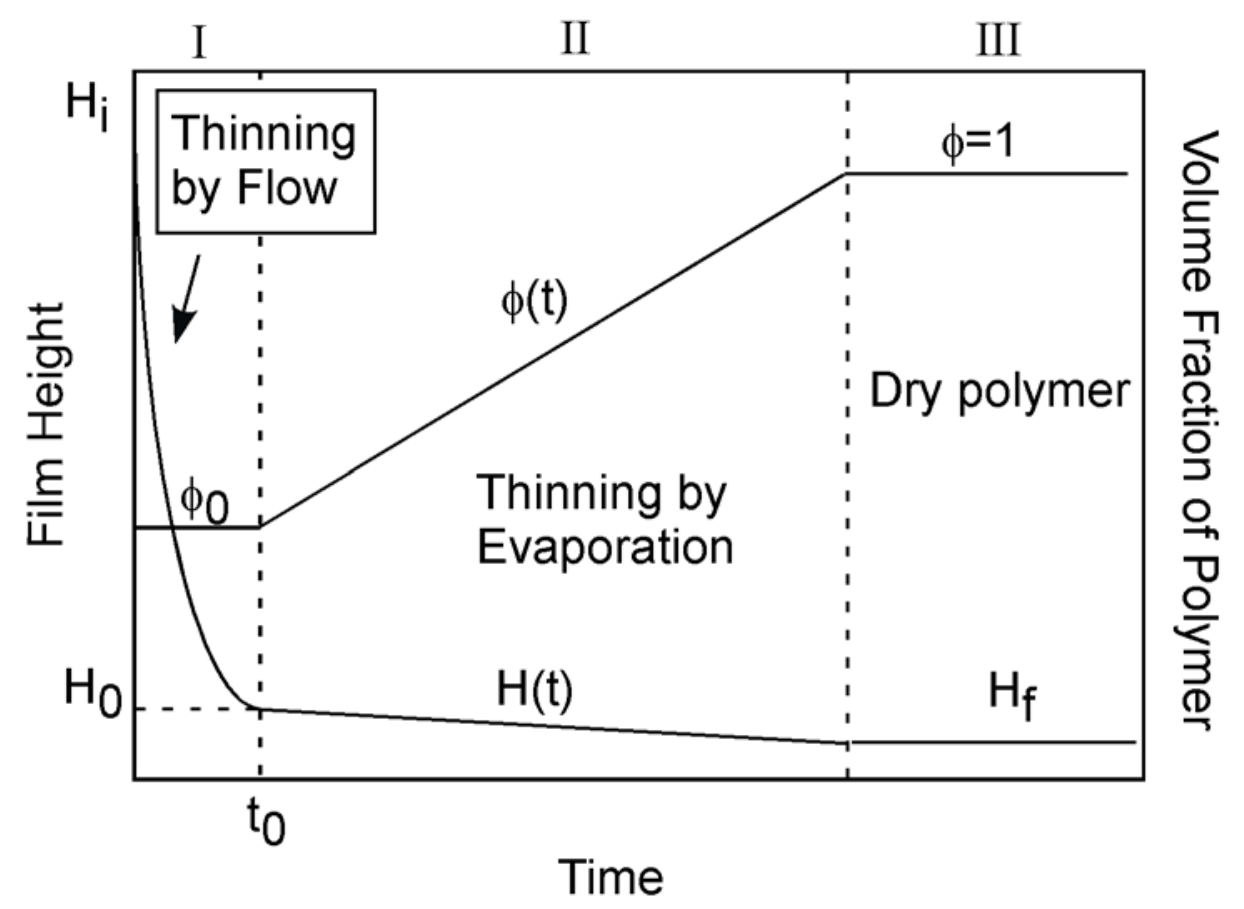

Figure 3.3. Different flow regimes in spin coating. 


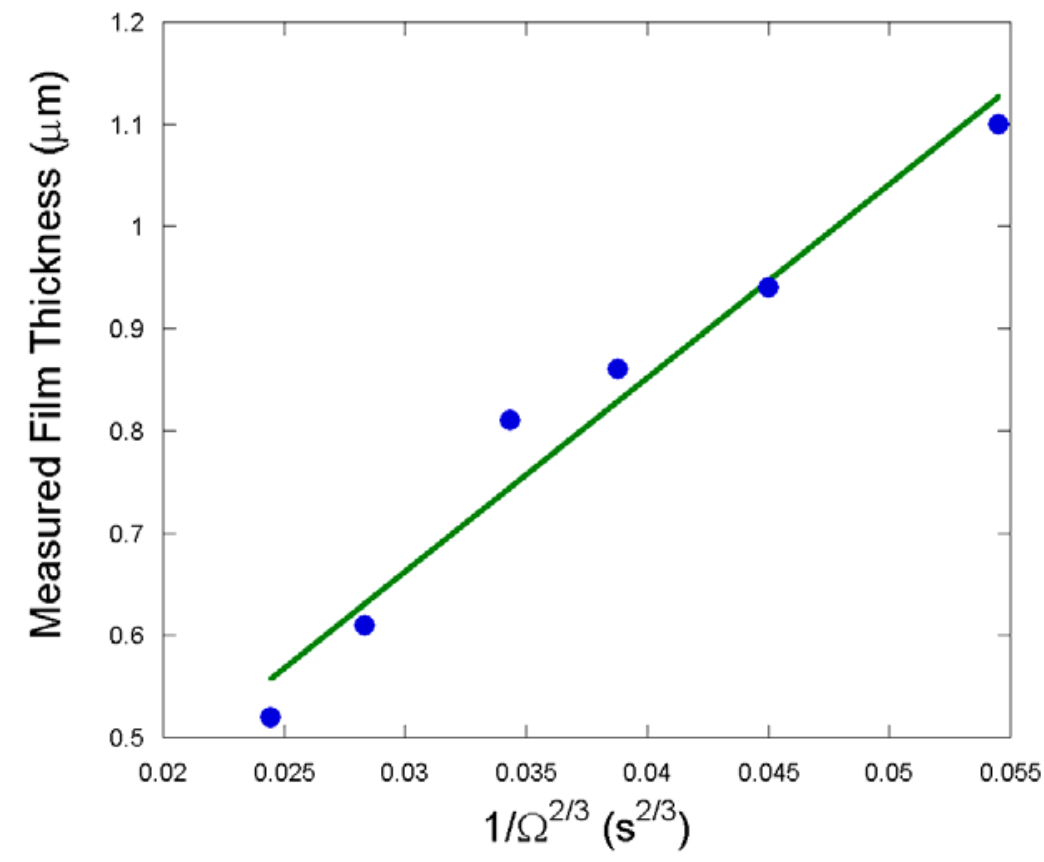

Figure 3.4. Measured film thickness vs $1 / \Omega^{2 / 3}$. 


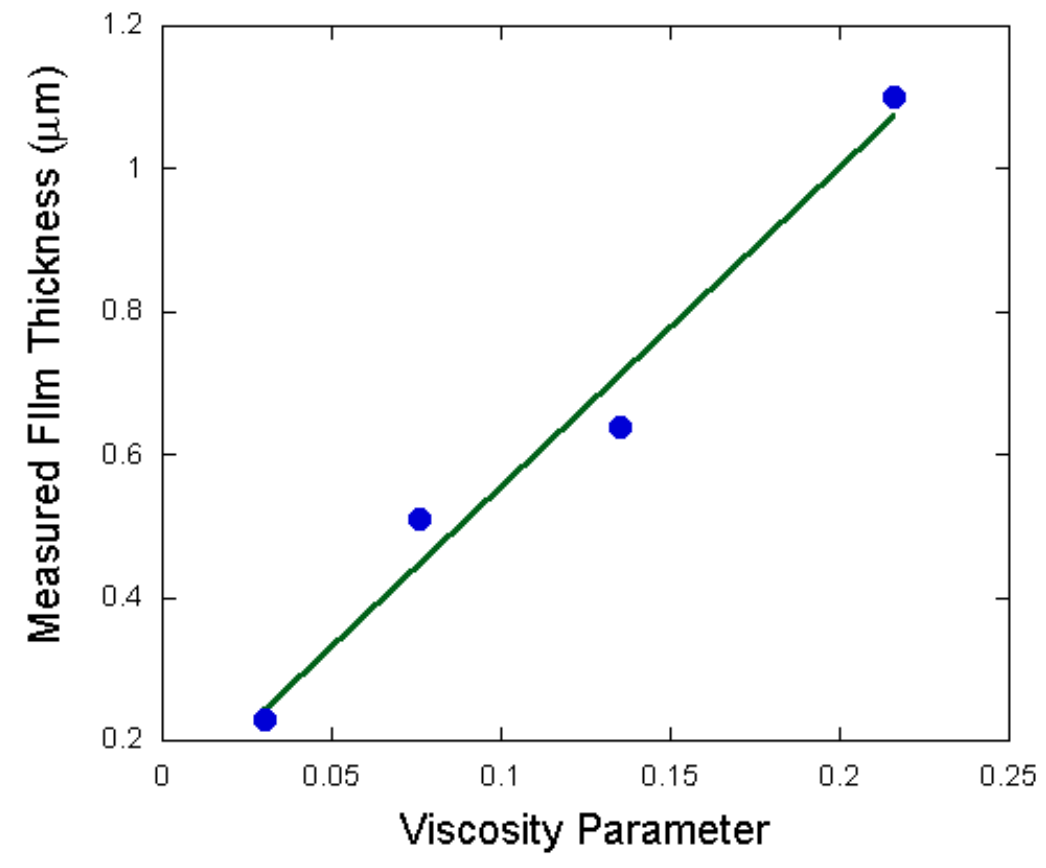

Figure 3.5. Measured film thickness versus $\varphi_{o}\left(\frac{\mu_{o}}{\left(1-\varphi_{o}\right)}\right)^{1 / 3}\left(\frac{k g}{m \cdot s}\right)^{1 / 3}$. 


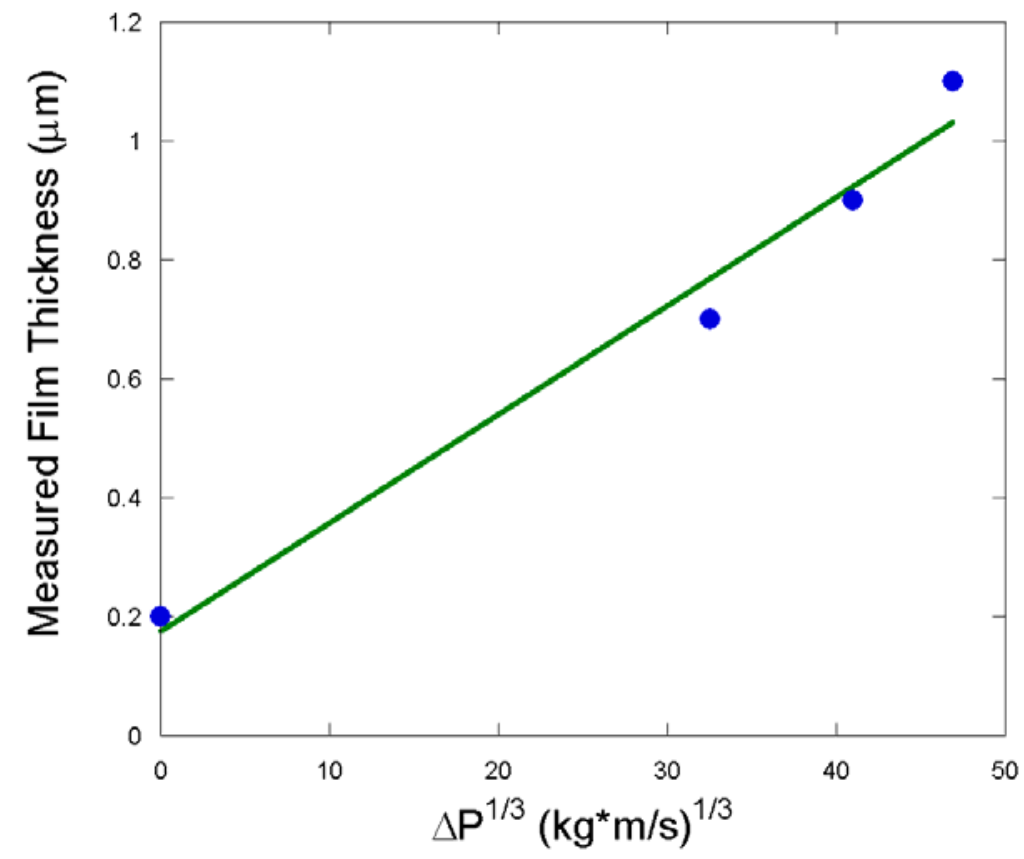

Figure 3.6. Measured film thickness vs $\Delta \mathrm{P}^{1 / 3}$. 

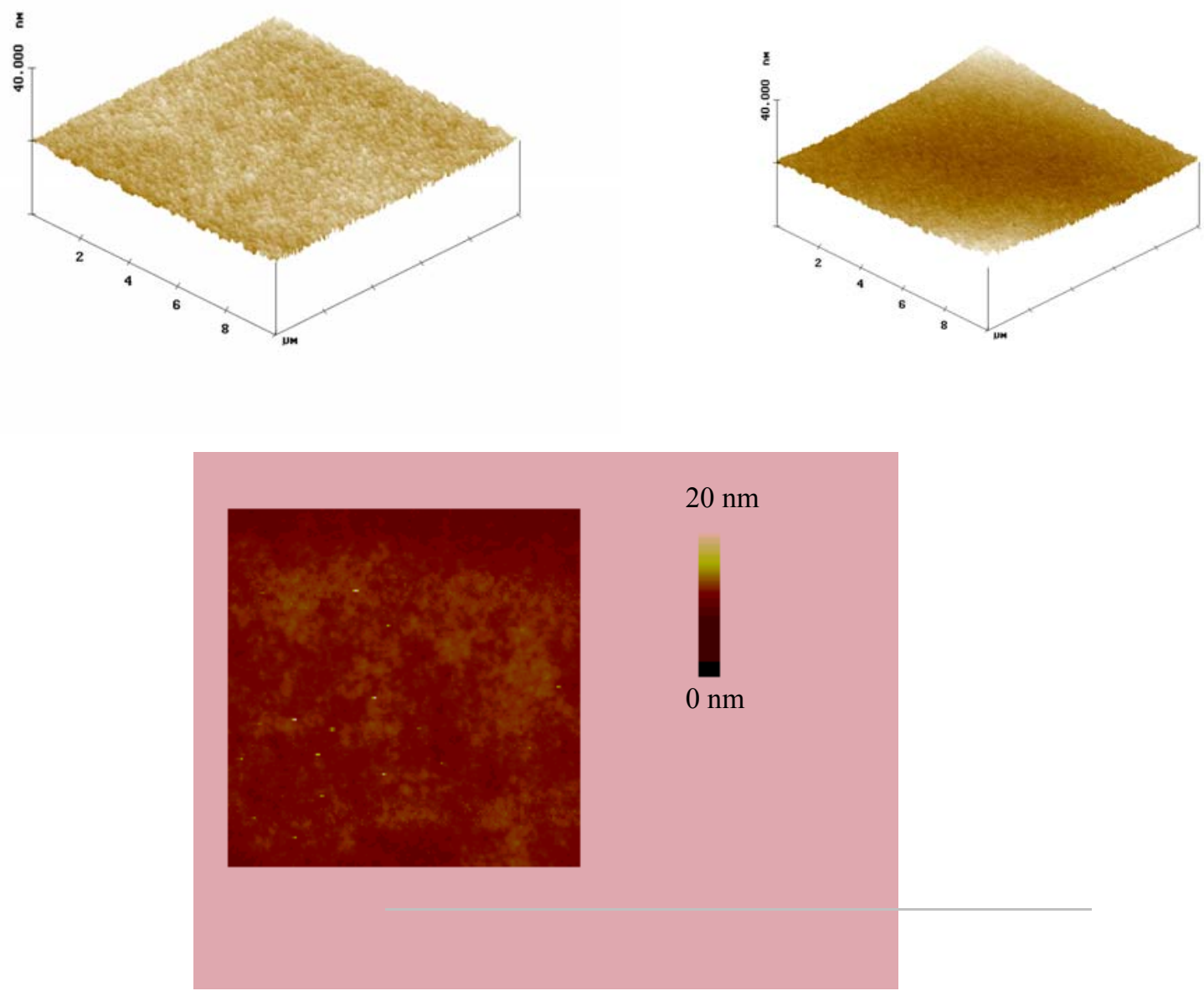

Figure 3.7. AFM scan of a photoresist film spun from $\mathrm{CO}_{2}(10 \times 10 \mu \mathrm{m})$ (Dario Goldfarb, IBM Corp, Yorktown Heights) 


\section{Chapter 4. Exposure and development of $\mathrm{CO}_{2}$ based photoresist}

\section{Deep-UV exposures - First Batch}

With lithographic quality spun cast films in hand, it became necessary to investigate the photolithographic performance of the potential photoresists. To that end, we began a series of exposures and developing experiments using a deep-UV flood exposure tool. The exposure system used was constructed by Cody Berger, a graduate student in Chemical Engineering at the Georgia Institute of Technology, under the supervision of Professor Clifford Henderson.

The core of the flood exposure system was a $500 \mathrm{~W} \mathrm{Hg}(\mathrm{Xe})$ flood exposure tool $(220-260 \mathrm{~nm})$ from Oriel. Coupled with a light intensity controller (Oriel 68851) and a $250 \mathrm{~nm}$ filter (Oriel 59580), we were able to generate sufficient photons at $250 \mathrm{~nm}$ to expose our films. The wafer was manipulated by placing it on a dual-axis linear stage (Daedal). An integrated computer control system (Bridgeview) regulated both the wafer movement and the shutter control. The entire apparatus was housed in a small cleanroom (Ultrapure) equipped with two high purity filters to prevent base contamination (Extraction Systems). Using this setup, it was possible to expose a 5 x 5 matrix of dosages on each wafer, or alternately, to flood expose the entire wafer to a single dosage through a photomask. 
The previous chapter discussed spin coating theory and film qualities obtained using a poly(FOMA-r-TBM) copolymer. Prior to the exposure work, attempts were made to improve upon the photoresist chemistry in order to improve performance. The poly(FOMA-r-TBM)polymers used exhibited a fairly low glass transition temperature ( $\mathrm{T}_{\mathrm{g}}$ $\sim 42{ }^{\circ} \mathrm{C}$ ). In addition, the t-butyl group, while behaving as an adequate leaving group, was not as easily cleavable as analogous compounds. Thus, to improve potential performance, an alternate copolymer was synthesized in preparation for the flood exposure experiments. The polymer used were random copolymers of poly(1,1dihydroperfluorooctyl)methacrylate 2-tetrahyrdopyranyl methacrylate (PFOMA-rTHPMA). This polymer exhibited a slightly higher $\mathrm{T}_{\mathrm{g}}$ and was more readily cleavable by the activated PAG, resulting in the same methacrylic acid as the TBM cleavage (Figure 4.1). Another benefit of using the THPMA copolymer is a reduction of swelling in $\mathrm{CO}_{2}$ compared to the TBM copolymer.

In the initial deep-UV exposure trials, it was noticed that the poly(FOMA-rTBM)polymer resin swelled to such a large extent that imaged features became extremely distorted. In order to rectify this problem, several new resins were synthesized, including the PFOMA-r-THPMA copolymers. To evaluate any decrease in swelling using the new resins, several experiments were performed in which a bulk sample of the polymer was exposed to $\mathrm{CO}_{2}$ and swelled over time.

These experiments were carried out in a high pressure $\mathrm{CO}_{2}$-view cell equipped with sapphire windows. This cell was similar to the cells discussed previously in this work, with the exception of its inner chamber, which was rectangular rather than 
cylindrical. To begin an experiment, a small amount $(\sim 500 \mathrm{mg})$ of resin was heated slightly above its Tg and placed in a small flat-bottomed glass vial designed to fit into the rectangular view cell. The samples were allowed to rest overnight to eliminate any large bubbles or voids. The vials were then placed into the view cell and the cell was pressurized. The swelling was observed over time by a CCD camera attached to a PC in the lab (Figure 4.2). The CCD camera was set to record images every 60 seconds over the course of 14 hours. After the set time was elapsed, the frames were then examined individually using the image acquisition software (Scion Image) and the height of the polymer was recorded as a function of time. Using this data, it was possible to plot the rise in polymer height as a function of time. Figure 4.3 shows one such plot.

Analysis of the data showed that the THPMA did indeed swell less than the TBM copolymers. As can be seen in Table 4.1, at a pressure of 1500 psi, the TBM copolymer swelled nearly $60 \%$ over the course of the experiment. This is significantly higher than the THPMA copolymer's 35\% swelling. This experiments indicated that the THPMA resin should indeed deform much less in $\mathrm{CO}_{2}$ processing. Also of note, the deprotected resin showed extremely small amounts of swelling. Thus swelling in exposed regions wafers should minimal. 
Table 4.1. Swelling of various copolymer resins over the course of 14 hours.

\begin{tabular}{cccc} 
Sample & $\begin{array}{c}\text { Initial } \\
\text { Height } \\
(\mathrm{mm})\end{array}$ & $\begin{array}{c}\text { Final } \\
\text { Height } \\
(\mathrm{mm})\end{array}$ & $\begin{array}{c}\text { Volume \% } \\
\text { swelling }\end{array}$ \\
\hline TBM at 1500 & 4.71 & 7.47 & $58.7 \%$ \\
THPMA at 1500 & 2.41 & 3.24 & $34.4 \%$ \\
Deprotected THPMA at 1500 & 2.15 & 2.22 & $3.3 \%$ \\
TBM at 2100 & 7.53 & 7.94 & $5.4 \%$ \\
THPMA at 2000 & 3.25 & 3.44 & $5.8 \%$ \\
Deprotected THPMA at 2000 & 2.22 & 2.22 & $0.0 \%$
\end{tabular}

Three different batches of this polymer were used in the first round of exposures as shown in Table 4.2.

Table 4.2. Copolymer resins used in initial flood exposure work.

\begin{tabular}{|c|c|c|c|c|c|}
\hline Sample & Target & FOMA & FOMA & $\mathbf{T}_{\mathbf{g}}$ & $\mathbf{T}_{\mathbf{d}}$ \\
\# CLM- & MW & Mole \% & Mole \% & (DSC) & (TGA) \\
A- & & (Feed) & (NMR) & & onset \\
\hline $\mathbf{0 0 3}$ & $5 \mathrm{~K}$ & 65 & 66 & - & 154.5 \\
\hline $\mathbf{0 0 9}$ & $25 \mathrm{~K}$ & 65 & 67 & - & 146.0 \\
\hline $\mathbf{0 1 5}$ & $25 \mathrm{~K}$ & 80 & 80 & 47.6 & - \\
\hline
\end{tabular}

Suitable films were spun of each of these photoresists, with film thicknesses shown in Table 4.3. Films were slightly thicker than necessary, but within an acceptable limit for 
the flood exposure tool. These films were then exposed to varying degrees of radiation dosage, according to the dose matrix given in Figure 4.4. A post exposure bake of 120 ${ }^{\circ} \mathrm{C}$ was used for all exposures. This PEB temperature was considerably higher than the $\mathrm{Tg}$ of the polymer, which is not optimal.

Table 4.3. Film thicknesses and PAG loadings.

\begin{tabular}{|c|c|c|c|}
\hline Sample \# & Polymer & $\begin{array}{c}\text { PAG } \\
\text { Concentration } \\
\text { (wt\% PAG) }\end{array}$ & $\begin{array}{c}\text { Film Thickness } \\
(\AA)\end{array}$ \\
\hline $\mathbf{3}$ & CLM-A-003 & 5 & 3970 \\
\hline $\mathbf{6}$ & CLM-A-015 & 4 & 11968 \\
\hline $\mathbf{7}$ & CLM-A-009 & 4 & 14114 \\
\hline $\mathbf{8}$ & CLM-A-009 & 4 & 14114 \\
\hline
\end{tabular}

After exposure, the films were sealed in single wafer shippers (Fluoroware) and transported back to the $\mathrm{CO}_{2}$ lab at NCSU. The films were sectioned and then developed in $\mathrm{CO}_{2}$. The $\mathrm{CO}_{2}$ development apparatus consisted of a 1" diameter high pressure tubular reactor, which was supplied with an inlet capable of delivering a steady stream of $\mathrm{CO}_{2}$ and equipped with a high pressure control valve (Badger) to regulate the $\mathrm{CO}_{2}$ effluent. In this manner, $\mathrm{CO}_{2}$ may be supplied at both a predetermined flowrate and a fixed pressure. Supercritical $\mathrm{CO}_{2}$ was supplied by a high pressure syringe pump (Isco) with a heating jacket. The developing chamber itself was also heated to prevent temperature fluctuations. The ends of the column were packed with glass wool to distrubte the $\mathrm{CO}_{2}$ 
flowing in and to prevent high sheer on the exposed surface. A rough of this apparatus is given in Figure 4.3. Using this apparatus, the exposed films were developed according to the conditions listed in Table 4.4.

Table 4.4. $\mathrm{CO}_{2}$ development conditions

\begin{tabular}{|c|c|c|c|}
\hline Sample \# & Polymer & $\begin{array}{c}\text { Development } \\
\text { Temperature }\left({ }^{\mathbf{}} \mathbf{C}\right)\end{array}$ & $\begin{array}{c}\text { Development } \\
\text { Pressure (psi) }\end{array}$ \\
\hline $\mathbf{3}$ & CLM-A-003 & 70 & 2050 \\
\hline $\mathbf{6}$ & CLM-A-015 & 70 & 2500 \\
\hline $\mathbf{7}$ & CLM-A-009 & 70 & 3300 \\
\hline $\mathbf{8}$ & CLM-A-009 & 70 & 3500 \\
\hline
\end{tabular}

All films were developed at $70{ }^{\circ} \mathrm{C}$. Pressures were varied according to the optimum for each polymer composition/molecular weight. Optimum conditions were determined by placing films of identical thickness into the developing chamber and identifying the lowest pressure at which the film was completely removed. Although samples 7 and 8 were of identical compositions, the pressure was increased slightly for sample 8 due to a small amount of residual polymer for sample 7. Raising the pressure 200 psi ensured that the unexposed areas were completely clean.

After development, the film thickness in each exposure region was measured using profilometry. The samples using the 003 and 015 polymers were of such low sensitivity that even $50 \mathrm{~mJ} / \mathrm{cm}^{2}$ was not sufficient to fully cleave the leaving groups. 
Because of this, the residual film thicknesses were relatively small and a normalized film thickness could not be obtained because the contrast curve never reached the asymptote. The partial curves obtained are shown in Figure 4.6.

Samples 7 and 8 were much more sensitive, producing complete sensitivity curves in both cases. The contrast in both cases was rather low, and the dosage to clear was still relatively high, as can be seen in Figure 4.7. It was clear, however, from these first experiments that there was much room for improvement. This improvement was realized in the second round of experiments.

\section{Deep-UV exposures - Improved Results}

The next round of flood exposure experiments focused on generating improved contrast curves, identifying the effect of various process variables, and generating some actual imaged films using $\mathrm{CO}_{2}$ development.

Building upon the successes of the first trials, all polymers used in the second round of experiments were PFOMA-r-THPMA in a 65:35 mol ratio. Three batches of different molecular weights were synthesized, designated A, B, and C. Sample B was estimated to be $15 \mathrm{k}$ MW, sample A to be $25 \mathrm{k} \mathrm{MW}$, and sample C to be $35 \mathrm{k}$ (was later

confirmed by GPC to be $39 \mathrm{k}$ MW). A battery of experiments was designed to investigate the effect of resin MW, PAG type (ionic or nonionic) and concentration (1\% or 5\%), and post exposure baking temperature $\left(90^{\circ} \mathrm{C}, 105^{\circ} \mathrm{C}, 120^{\circ} \mathrm{C}\right)$. A summary of the various combinations investigated is given in Table 4.5. 
The experimental procedures in the second round were quite similar to those presented previously. The equipment and exposure matrices used were identical. Significant differences include the batch-to-batch variability associated with the polymer synthesis method, in which much greater care was taken to ensure the samples were fresh and uncontaminated, and the timing of the experiments, where every effort was taken to eliminate the delay between the spin coating and exposure of the films. It is anticipated that these samples had a broad chemical composition distribution as well. This issue will be explored in later work when synthesis is converted from a batch process to a continuous polymerization.

The first variable examined was the effect of the resin MW. Three separate wafers were exposed with a PEB of $90{ }^{\circ} \mathrm{C}$ and $5 \%$ ionic PAG loading, but using each of the three different resins. Sensitivity plots for these three samples are given in Figure 4.8. The highest MW resin (in green) clearly has a much poorer contrast than the other two MW samples. The contrast, $\gamma$, for the $35 \mathrm{k}$ MW sample was 1.2 , as opposed to a $\gamma$ of 3.3 and 3.6 for the $15 \mathrm{k}$ and $25 \mathrm{k}$ MW samples respectively. However, the trend in sensitivities is less apparent, with the $25 \mathrm{k}$ MW sample being the most sensitive, while the $15 \mathrm{k} \mathrm{MW}$ resin takes as much dose to clear as the $35 \mathrm{k}$ MW resin. 
Table 4.5. Experimental conditions used in the second round of deep-UV exposures.

\begin{tabular}{|c|c|c|c|c|c|c|}
\hline Sample \# & Resin & PAG Loading & PEB & Images & Contrast & Sensitivity \\
\hline 1 & B & $1 \mathrm{wt} \%$ IP & $90 \mathrm{C}$ & 13 faint & - & - \\
2 & B & $5 \mathrm{wt} \%$ IP & $90 \mathrm{C}$ & 25 clear & 3.6 & 19 \\
3 & C & $1 \mathrm{wt} \%$ IP & $105 \mathrm{C}$ & 14 faint & - & - \\
4 & C & $5 \mathrm{wt} \%$ IP & $90 \mathrm{C}$ & 25 clear & 1.2 & 19 \\
5 & A & $1 \mathrm{wt} \%$ IP & $105 \mathrm{C}$ & 15 faint & - & - \\
6 & A & $5 \mathrm{wt} \%$ IP & $105 \mathrm{C}$ & 25 clear & 3.3 & 12 \\
10 & C & $5 \mathrm{wt} \%$ IP & $105 \mathrm{C}$ & 25 clear & 2.6 & 17 \\
11 & C & $5 \mathrm{wt} \%$ IP & $120 \mathrm{C}$ & 25 clear & 2.4 & 19 \\
\hline
\end{tabular}


The next variable investigated is the PEB temperature. Three wafers coated with resin $\mathrm{C}$ and 5\% ionic PAG were baked at three separate temperatures to determine the optimum PEB. As can be seen from Figure 4.9, all of the curves are fairly similar. However, the wafer baked at $90{ }^{\circ} \mathrm{C}$ clearly has a lower sensitivity than the other two wafers. This is counterintuitive and in contradiction to results presented by Ober, et al., where they found that raising the PEB acted to increase resist sensitivity [1]. One possible explanation for this phenomenon is the low $\mathrm{Tg}$ of these materials $\left(<50^{\circ} \mathrm{C}\right)$. By performing the PEB at a temperature much higher than the Tg of the material, it could be that acid diffusion is increased to such high levels that poorer sensitivity is observed.

The final variable investigated was the PAG chemistry. Two different PAGs were used in these experiments, one ionic and one nonionic (Figure 4.1a \& 4.1b). Exposures were performed using identical formulations save for the PAG. Figure 4.10 shows the contrast curves obtained. The nonionic PAG produces the highest contrast to date, however, the sensitivity is so poor that it is impractical for most applications. 30 $\mathrm{mJ} / \mathrm{cm}^{2}$ of radiation is required before the nonionic PAG exhibits any change at all. The ionic PAG, on the other hand, has a much lower dosage to clear, at the expense of a lower contrast. The reason for this trend was not entirely clear until subsequent UV-Vis experiments measured the absorbance of each PAG. By casting thin films of the resin/PAG and resin alone, it was possible to subtract the spectra to obtain absorbance data for the PAG in a thin film using a UV-Vis spectrophotometer (Jasco V-550). As can be seen in Figure 4.11, although both PAGs absorb radiation at $250 \mathrm{~nm}$, the nonionic 
absorption is an order of magnitude less, thus requiring far higher dosages to effect the release of the acid.

\section{Etch Resistance}

A key property of any photoresist is its ability to resist chemical and plasma etching. A detailed investigation of the etch resistance of $\mathrm{CO}_{2}$-soluble photoresists is beyond the scope of this work. However, a few experiments were conducted as part of this research.

It was anticipated that the PFOMA-r-THPMA polymer alone would have relatively poor etch resistance. To improve upon that resistance, additional comonomers were added to the resin and their effect was evaluated. It is known that siloxane containing polymers form $\mathrm{SiO}_{2}$ upon exposure to the etching plasma, which then serves are a barrier to etching. Therefore, several siloxane containing groups were copolymerized with the PFOMA-r-THPMA resin to improve their etch resistance. The synthesis of these materials is shown in Figure 4.12. The additional groups were only added in small concentrations $(5 \%, 10 \%$, and $20 \%)$ in order to minimize their effect on the contrast and sensitivity of the current resists.

The polymers were synthesized as shown, and then spun onto 6" wafers in thin films. After spinning, the resists were thermally deprotected. The polymers were then etched with $25: 75 \mathrm{v} / \mathrm{v} \mathrm{O}_{2}: \mathrm{SF}_{6}$ in a parallel plate RIE $(13.0 \mathrm{MHz})$ at $20^{\circ} \mathrm{C}, 60 \mathrm{mTorr}$, and 100W. For comparison, the base resin was also spun and etched, as well as a commercial novolac based photoresist (Shipley 1813). The results of these experiments are shown in Table 4.6. 
Table 4.6. Etching rates of several photoresist formulations.

\begin{tabular}{|c|c|c|c|c|}
\hline Photoresist & Avg. Etch Rate (A/min.) & mol\% FOMA & mol\% THPMA & mol\% MPTS or PDMSMA \\
\hline Shipley 1813 (novolac) & 562 & 0 & 0 & 0 \\
\hline p(FOMA-r-THPMA) & 1405 & 65 & 35 & 0 \\
\hline p(FOMA-r-THPMA-r-PDMSMA) & 1388 & $\mathrm{NA}$ & $N A$ & $\mathrm{NA}$ \\
\hline p(FOMA-r-THPMA-r-MPTS) & 1262 & 65 & 28 & 7 \\
\hline p(FOMA-r-THPMA-r-MPTS) & 1272 & 54 & 33 & 13 \\
\hline p(FOMA-r-THPMA-r-MPTS) & 1312 & 43 & 30 & 27 \\
\hline
\end{tabular}

As can be seen in Table 4.6, the PFOMA-r-THPMA etches far quicker than the commercial resist. Adding the PDMSMA increased the etch resistance slightly, although it was difficult to obtain precise measurements as that polymer did not produce uniform spun films. Increasing the fraction of MPTS also served to increase the etch resistance of the polymer.

Few conclusions can be drawn from these limited experiments. The novolac resin used is a negative resist from an older technology node, and thus direct comparison of resist rates may not be possible. However, one clear conclusion is that the addition of siloxane groups does indeed increase the etch resistance of the $\mathrm{CO}_{2}$-soluble resin. This will be of great importance as work proceeds and additional research is conducted to tailor these initial resists for use in commercial photolithography.

\section{SEM images}


With the sensitivity plots in hand, it was possible to expose wafers at a suitable dosage to obtain patterned images. A photoresist formulation composed of the $25 \mathrm{k}$ MW resin and $5 \%$ ionic PAG was spun on a wafer and flood exposed at $248 \mathrm{~nm}$ radiation. For ease of processing, simple contact printing was used, wherein the photomask is placed directly on top of the coated wafer. Exposure dosages of $20 \mathrm{~mJ} / \mathrm{cm}^{2}$ were used. The exposed wafers were developed in supercritical $\mathrm{CO}_{2}$ at $60{ }^{\circ} \mathrm{C}$ and 4400 psi. After development, the sectioned wafers were examined using field emission scanning electron microscopy (JEOL 6400F SEM). Several of these micrographs are shown in Figure 4.12. As can be seen in the figure, we were indeed successful in producing images in $\mathrm{CO}_{2}$. The images appear sharp and well defined. However, due to limitations in the flood exposure tool and the photomasks available, the features were relatively large compared to current photolithographic standards. The smallest features produced were no smaller than $2 \mu \mathrm{m}$. But these images conclusively demonstrated that it was indeed possible spin coat and develop images using only liquid and supercritical $\mathrm{CO}_{2}$.

\section{Dissolution Rate Experiments}

In order to better understand the mechanism of dissolution in development, a series of experiments were begun in conjunction with Mohamed Elbaccouch. These experiments were designed to measure and model the dissolution of photoresist films from silicon wafers in a spinning geometry. Although the current development apparatus uses a flow through chamber, spinning and puddle development schemes are likely improvements upon the $\mathrm{CO}_{2}$ development work presented in this thesis. In addition, the fluid mechanics 
of a rotating disk are well understood and it is a useful geometry for measuring mass transfer coefficients.

The apparatus used in this work consists of a large pressure chamber designed to withstand pressure of $6000 \mathrm{psi}$ at $150^{\circ} \mathrm{C}$. Inside the chamber is a rotating holder upon which coated wafers may be affixed. The chamber can accommodate 4" wafers, although smaller wafers were often used to conserve material. The wafer holder was connected to a DynaMag high-pressure drive unit (Pressure Products) capable of rotational speeds up to $2500 \mathrm{rpm}$. The chamber was equipped with a heating and cooling jacket capable of operating at temperatures from $-10{ }^{\circ} \mathrm{C}$ to $150{ }^{\circ} \mathrm{C}$. The unit was also contained various inlet and outlet ports, a pressure transducer, a thermocouple, and motor controls as shown in Figure 4.13.

In this circular geometry, the shear stress acting on the film is

$$
\tau_{r z}=0.51 \mu r v^{-1 / 2} \Omega^{3 / 2}
$$

where $\tau_{\mathrm{rz}}$ is shear stress on the disk surface, $\mu$ is the fluid viscosity, $r$ is the radial position on disk surface, $v$ is the fluid kinematic viscosity, and $\Omega$ is the rotational speed of disk. If the film removal is limited by the mechanical force exerted on the surface, the film thickness as a function of time would be proportional to this shear stress. If, however, the dissolution is limited by diffusion, then the film removal will be proportional to the mass transfer coefficient, $R=k_{m}\left(C^{*}-C_{\infty}\right)$ (Figure 4.15). For a rotating disk, the mass transfer coefficient is [2] 


$$
k_{m}=0.6205 v^{-1 / 6} D^{2 / 3} \Omega^{1 / 2},
$$

where $\mathrm{D}$ is the diffusion coefficient, given by,

$$
D=1.173 \times 10^{-16}\left(\varphi M_{B}\right)^{1 / 2} \frac{T}{\mu_{B} V_{A}^{0.6}}
$$

and $\varphi$ is the association parameter ( 1 for $\mathrm{CO}_{2}$ ), $M_{B}$ is the molecular weight of $\mathrm{CO}_{2}$ ( $44.01 \mathrm{~g} / \mathrm{mole}$ ), $\mathrm{T}$ is the temperature in Kelvins, $\mu_{B}$ is viscosity of $\mathrm{CO}_{2}, V_{A}$ is the solute molar volume at the boiling point $\left(0.625 \mathrm{~m}^{3} / \mathrm{Kg}\right.$ mole for poly(FOMA-r-THPMA)). Much of the work on this apparatus to date has dealt with refinements to the equipment and development of a robust experimental procedure. Some of the initial data are given in Figure 4.16. Figure 4.17 is a plot of the film removal (for runs of the same length) plotted versus the square root of the rotational speed. The linear nature of these data indicates that the process is indeed diffusion limited. This early work is promising, but further experiments are necessary to make definitive conclusions about the mechanism of film dissolution during development.

With resist performance quantified and some preliminary images in hand, efforts then turned toward producing higher quality images using an industrial $193 \mathrm{~nm}$ stepper. These efforts are described in the following chapter. 


\section{References}

1. Sundararajan, N., et al., Supercritical CO2 Processing for Submicron Imaging of Fluropolymers. Chem. Mater., 2000. 12(1): p. 41.

2. Geankoplis, C., Transport Processes and Unit Operations. 3rd ed. 1993, Englewood Cliffs, NJ: Prentice Hall. 
a)<smiles>c1ccc([I-]c2ccccc2)cc1</smiles>

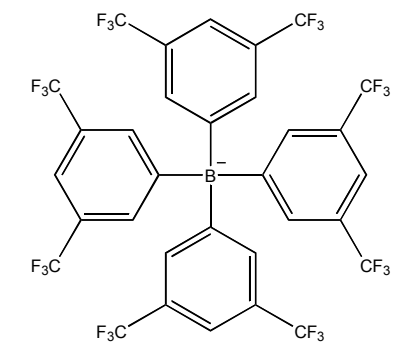

b)

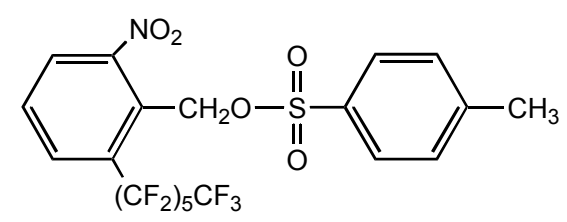

c)

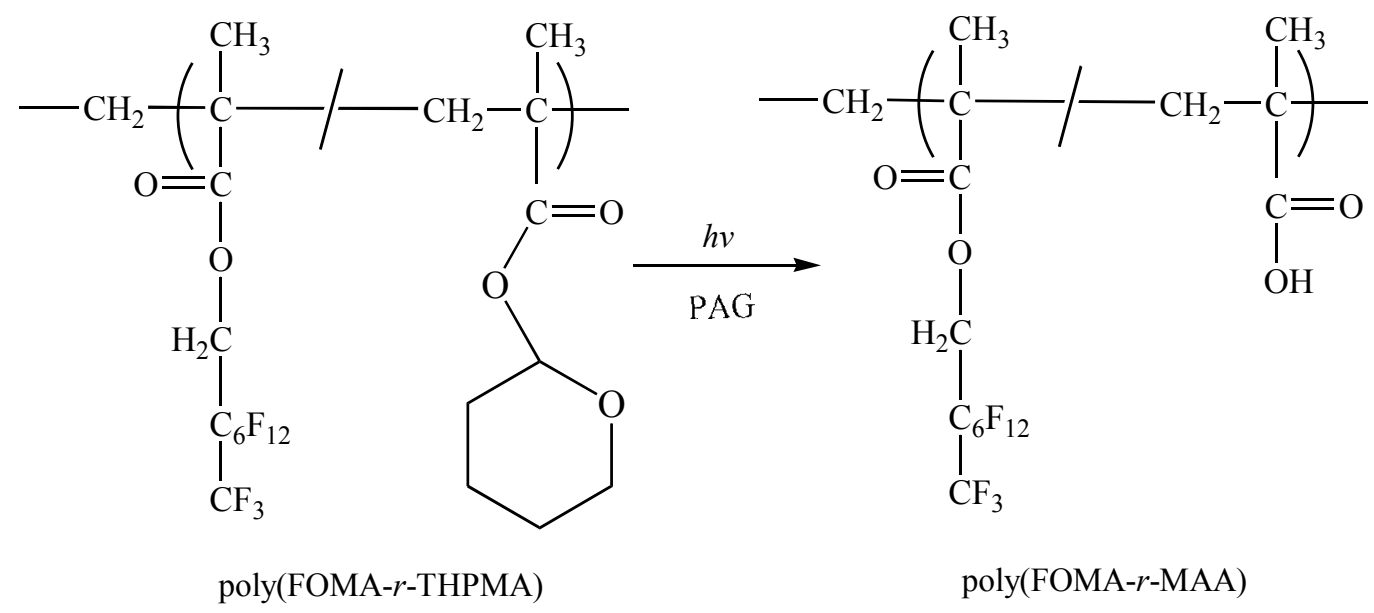

Figure 4.1. Structure of $\mathrm{CO}_{2}$ soluble photoresist system: a) ionic PAG, Diphenyliodonium tert-(3,5-bis(trifluoromethyl) phenyl boronium, b) nonionic PAG, 2-perfluorohexyl-6nitrobenzyl tosylate, c) $\mathrm{CO}_{2}$ soluble resin, poly(1,1-dihydroperfluorooctyl)methacrylate 2tetrahyrdopyranyl methacrylate. 


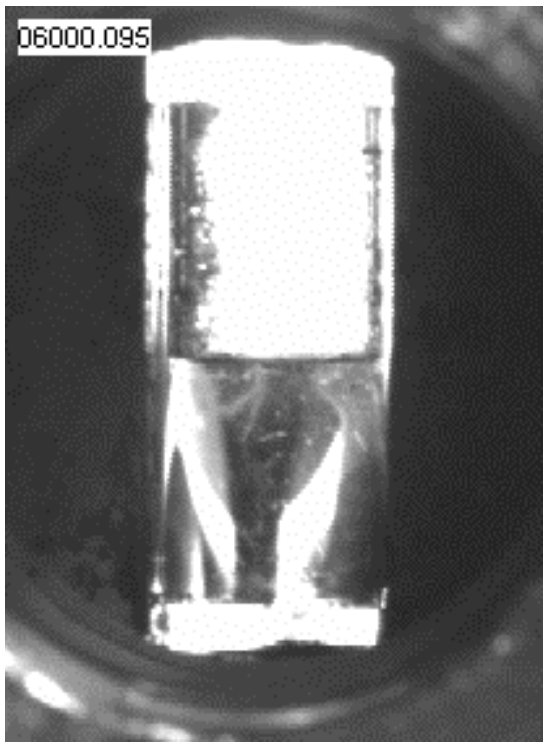

Figure 4.2. Sample frame measuring the swelling of TBM copolymer resins in $\mathrm{CO}_{2}$ at 1500 psi. 


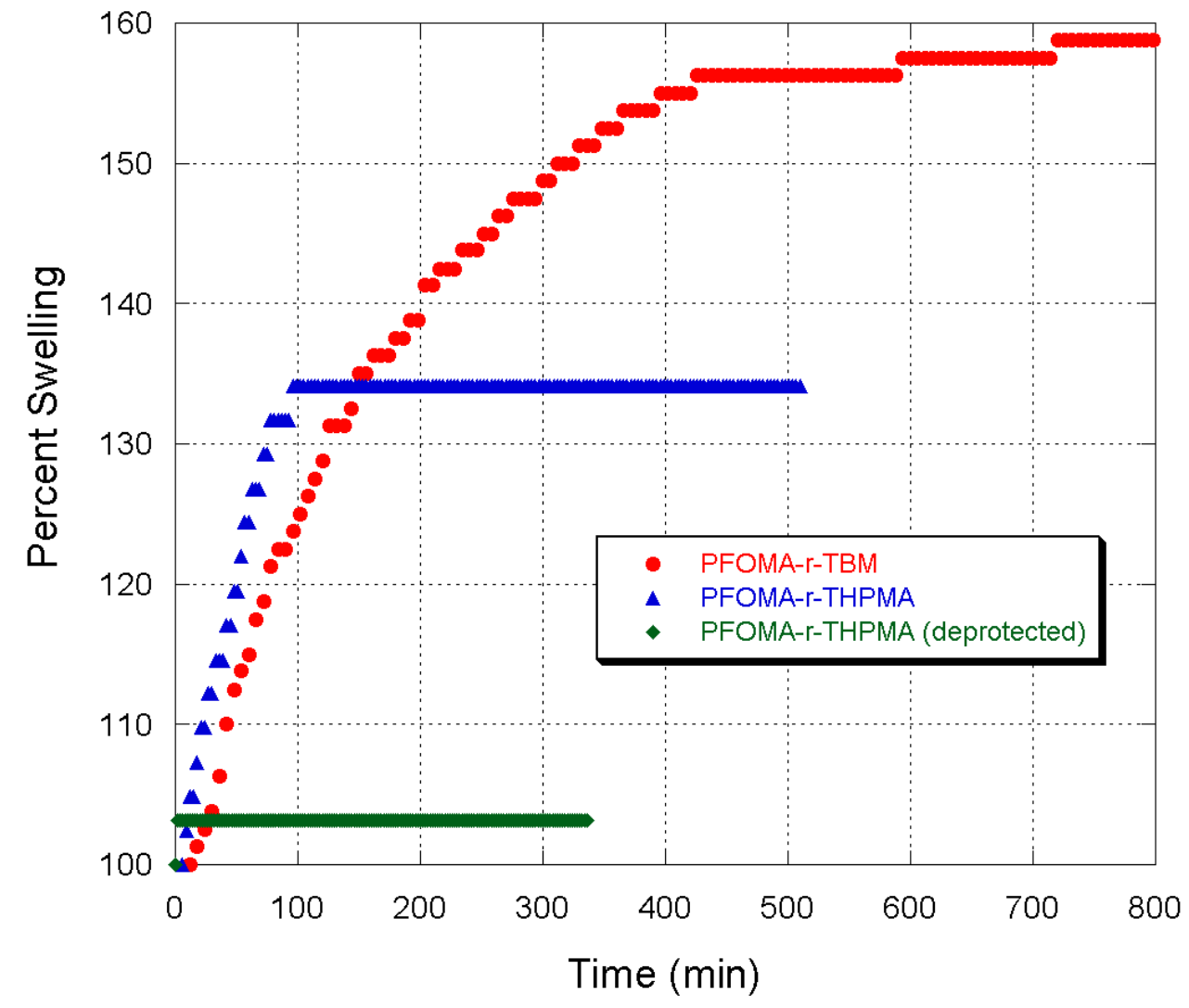

Figure 4.3. Swelling of three polymer resins in $\mathrm{CO}_{2}$ at $1500 \mathrm{psi}$. 


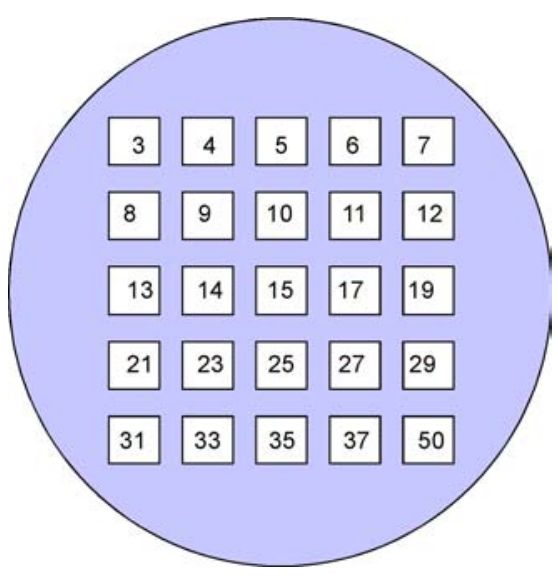

Figure 4.4. Dose matrix (with values in $\mathrm{mJ} / \mathrm{cm}^{2}$ ) used for samples $3,6, \& 7$. Sample 8 was exposed to a more narrow range, with dosages of $10-34 \mathrm{~mJ} / \mathrm{cm}^{2}$ in increments of 1 . 
a)

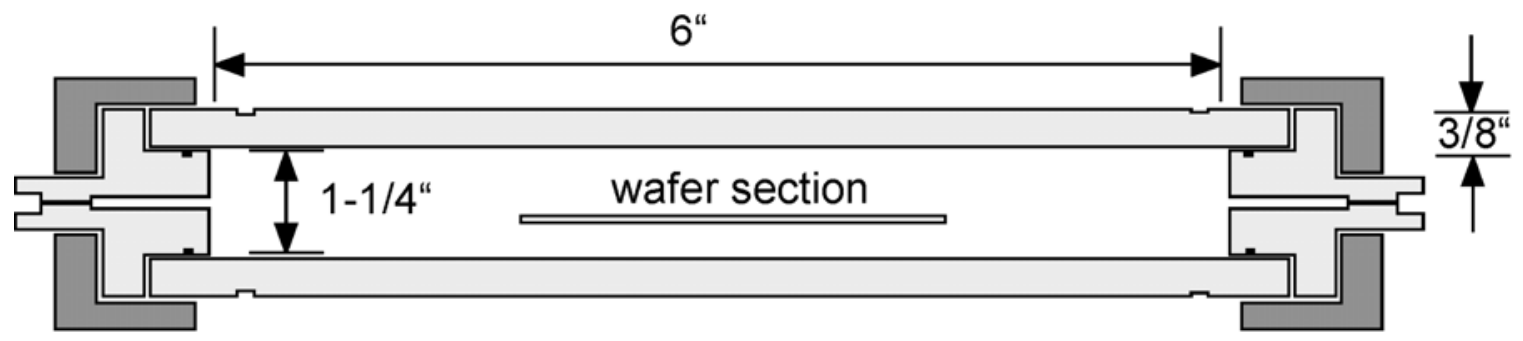

b)

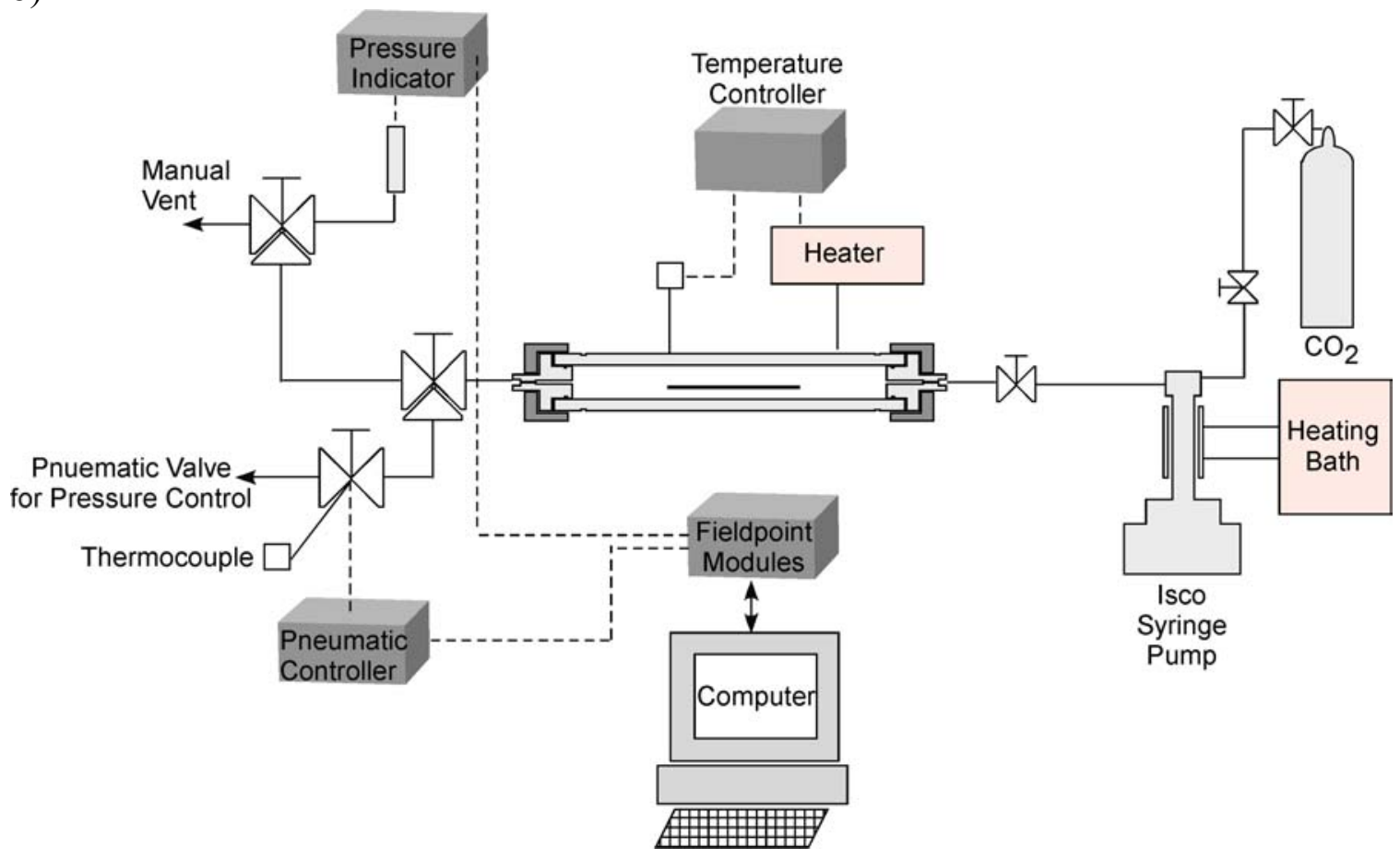

Figure 4.5. Schematic of the $\mathrm{CO}_{2}$ development chamber; a) development chamber; $b$ ) overview of development apparatus. High pressure tubing was a combination of $1 / 8$ " and 1/16" tubing (HiP). Manual valves (HiP), on/off pneumatic valves (HiP), and pneumatic control valves (Badger Meter) were used. Computer control was programmed with Bridgeview, and I/O between the computer, input and control devices was accomplished via Fieldpoint modules (National Instruments). 

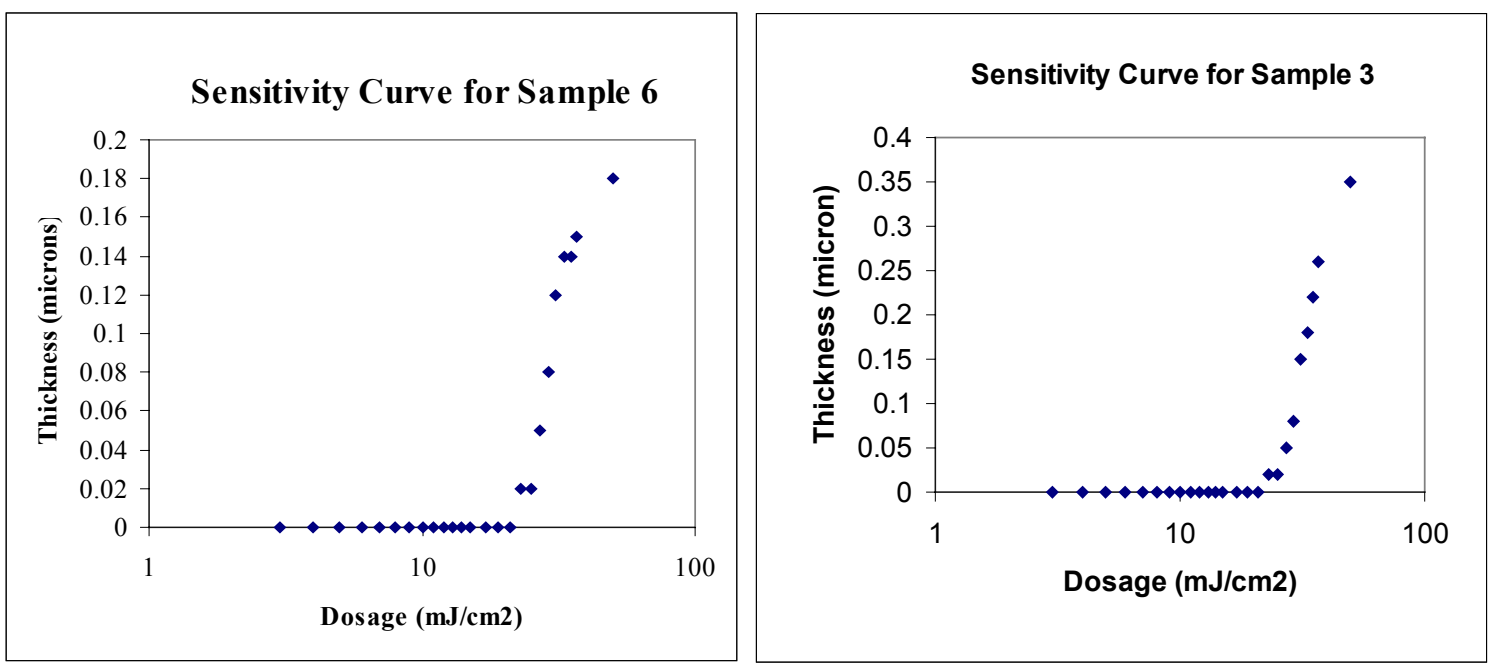

Figure 4.6. Sensitivity curve for sample 6, CLM-A-015 w/ 5\% ionic PAG and sample 7, CLM-A-003 w/ 3\% ionic PAG. 

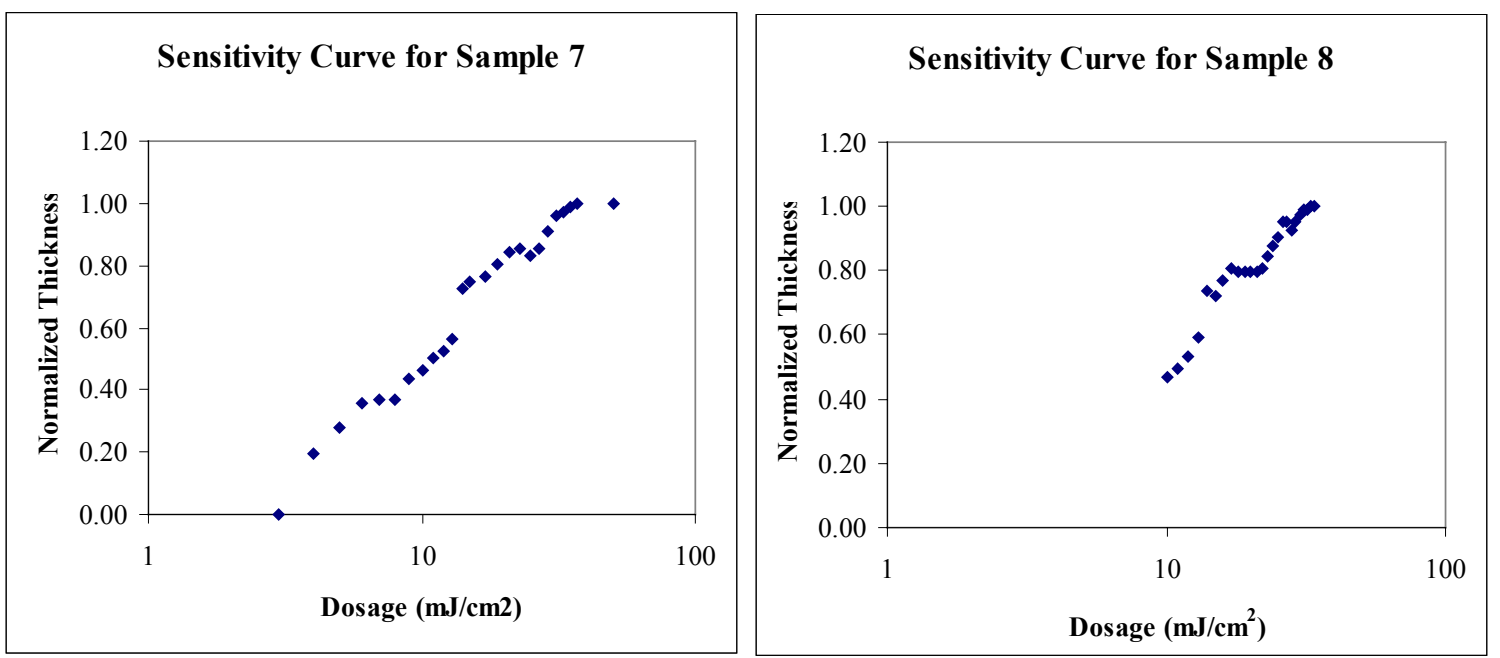

Figure 4.7. Sensitivity curve for sample 7, CLM-A-009 w/ 4\% ionic PAG, and sample 8, CLM-A-009 w/ 4\% ionic PAG. 


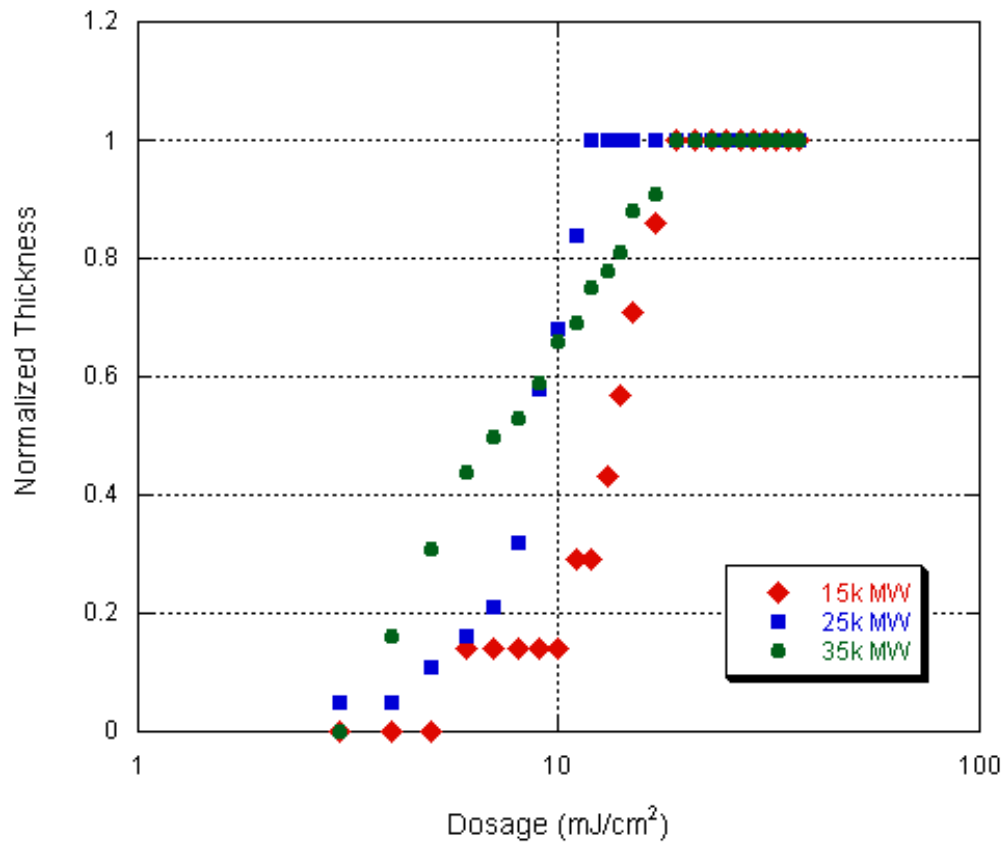

Figure 4.8. Sensitivity plots for three different MW resins at 5\% ionic PAG loading, PEB of $90^{\circ} \mathrm{C}$. 


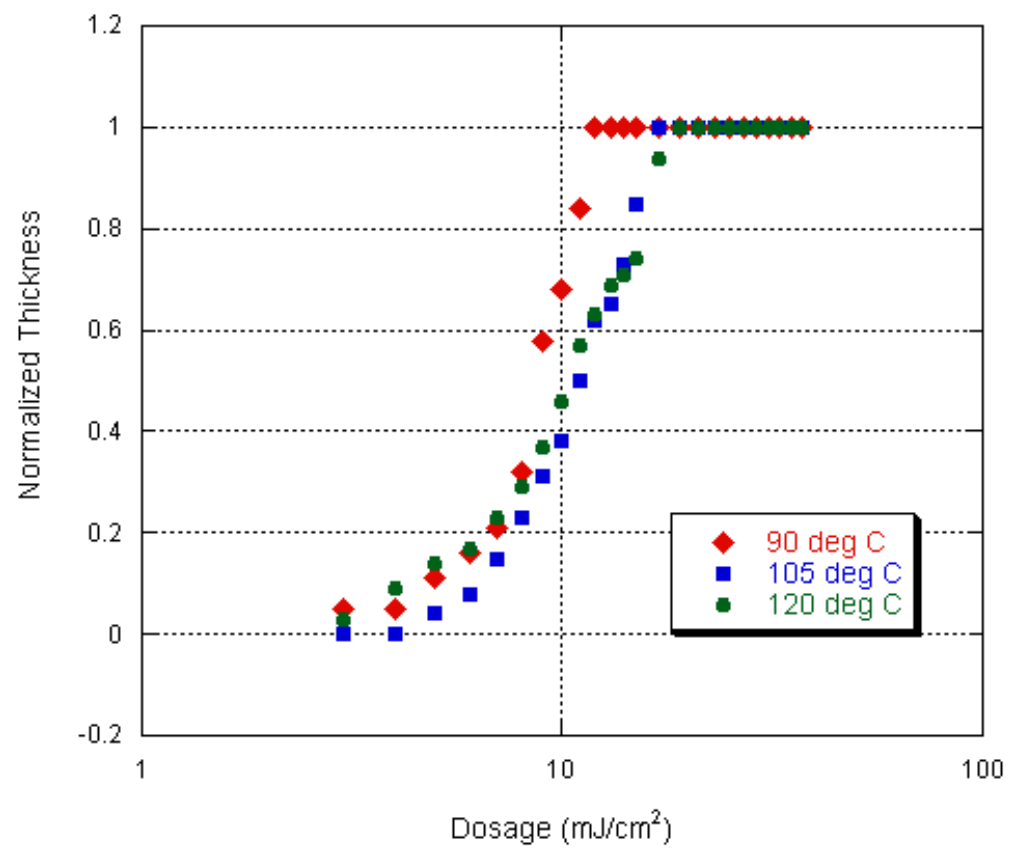

Figure 4.9. Sensitivity plots for three different PEB temperatures. All films were of resin $\mathrm{C}$ at $5 \%$ ionic $\mathrm{PAG}$ loading. 


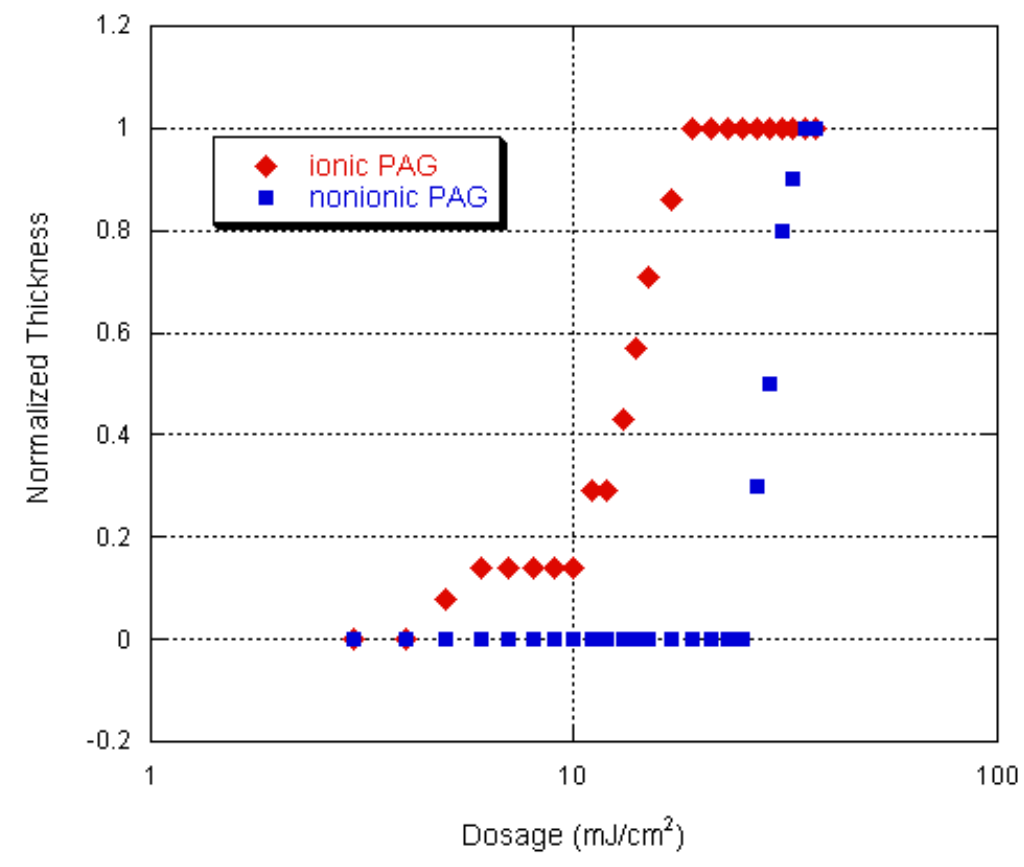

Figure 4.10. Sensitivity plot of resin $\mathrm{B}$, with $5 \%$ ionic $\mathrm{PAG}$ and $5 \%$ nonionic PAG. 

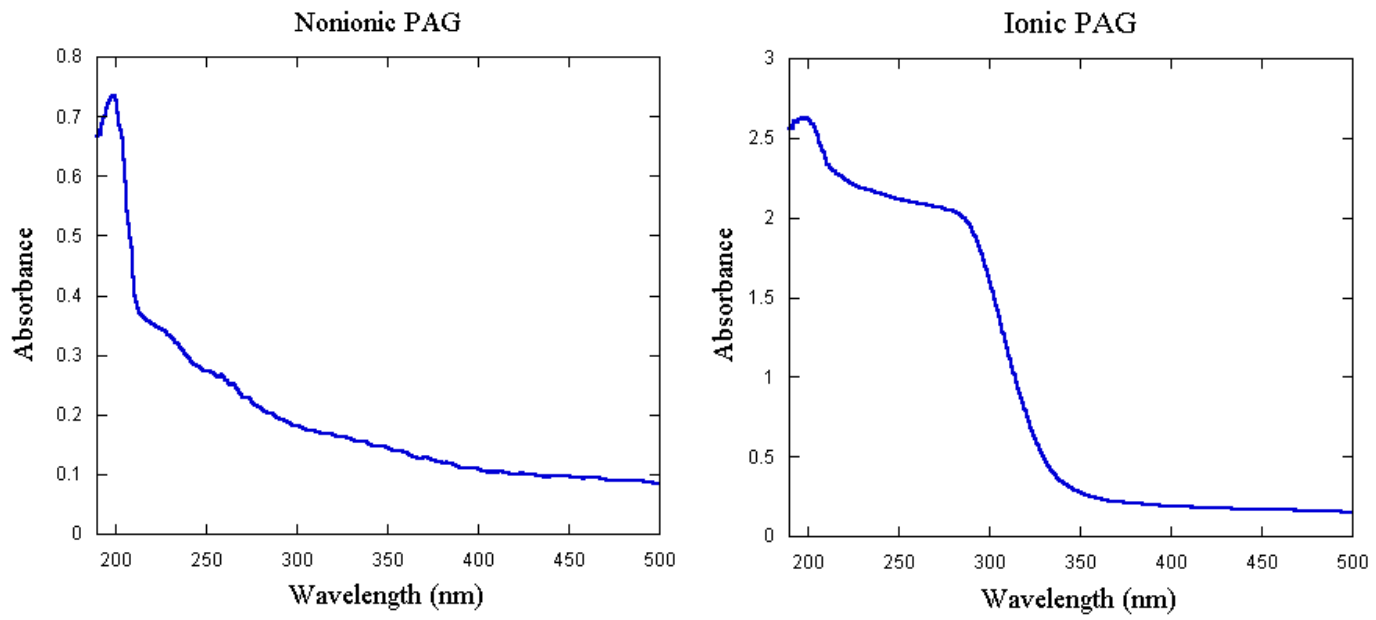

Figure 4.11. UV-Vis absorbance of the two photoacid generators. 

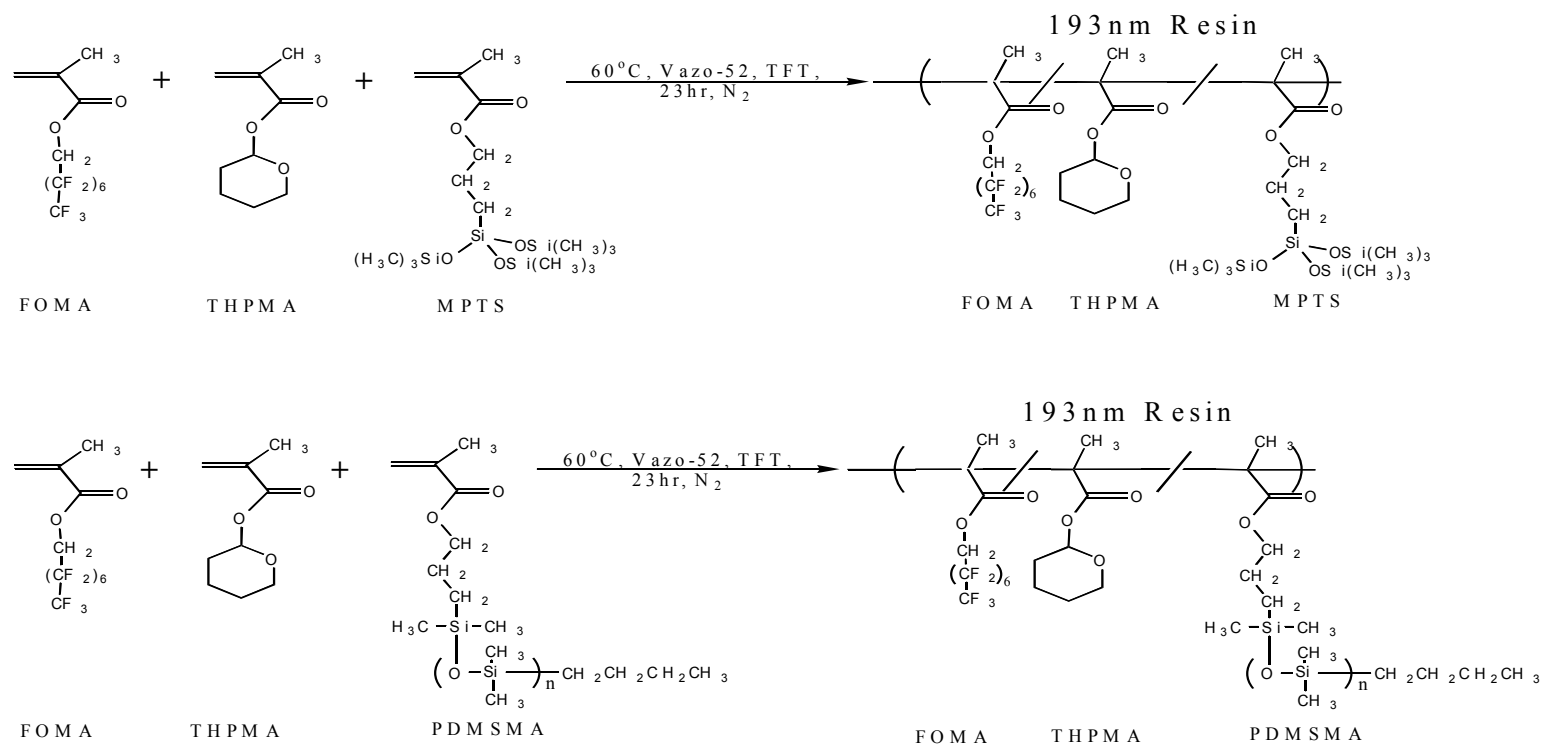

Figure 4.13. Comonomers investigated for etch resistance. 

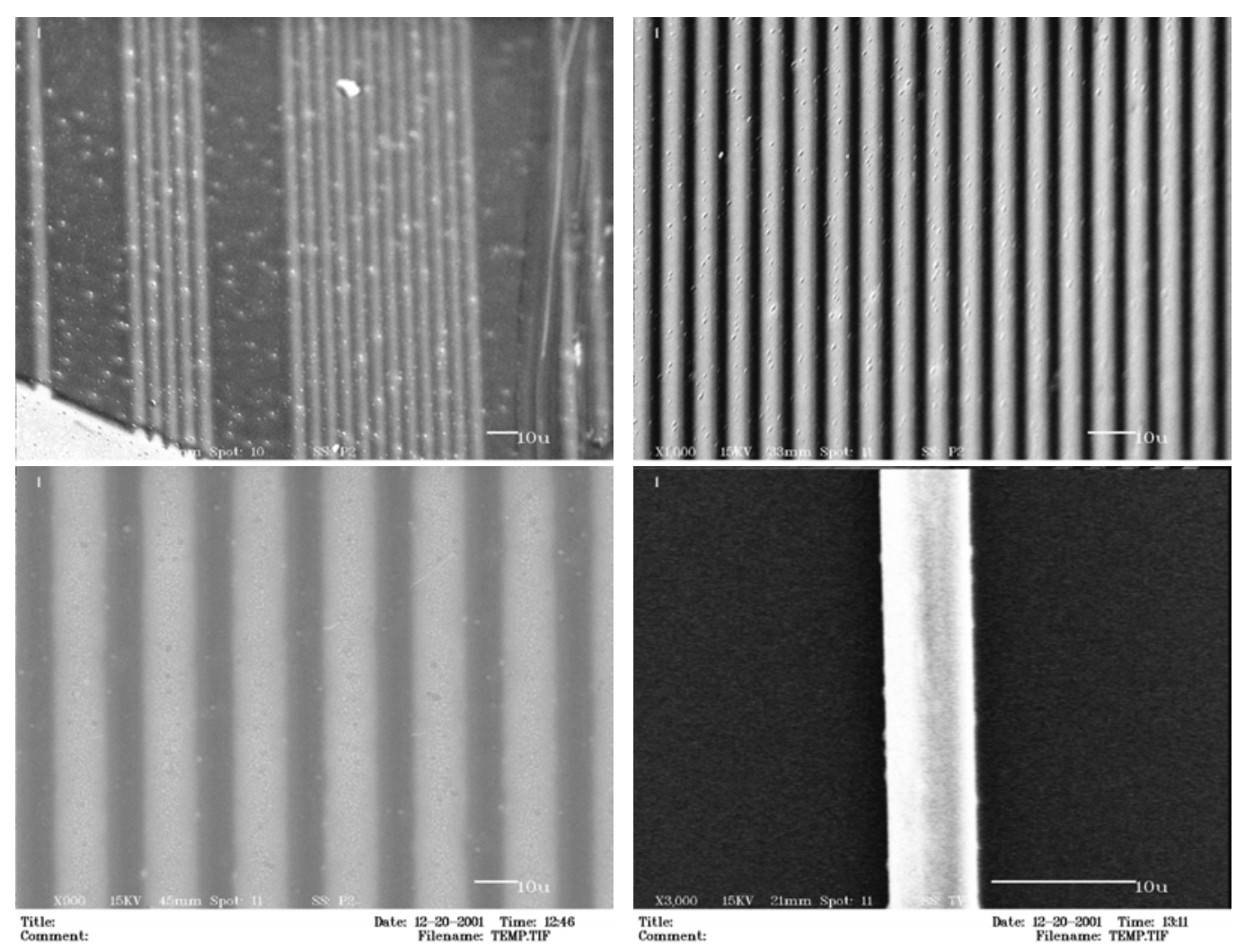

Figure 4.12. SEM images of $248 \mathrm{~nm}$ exposed features. 


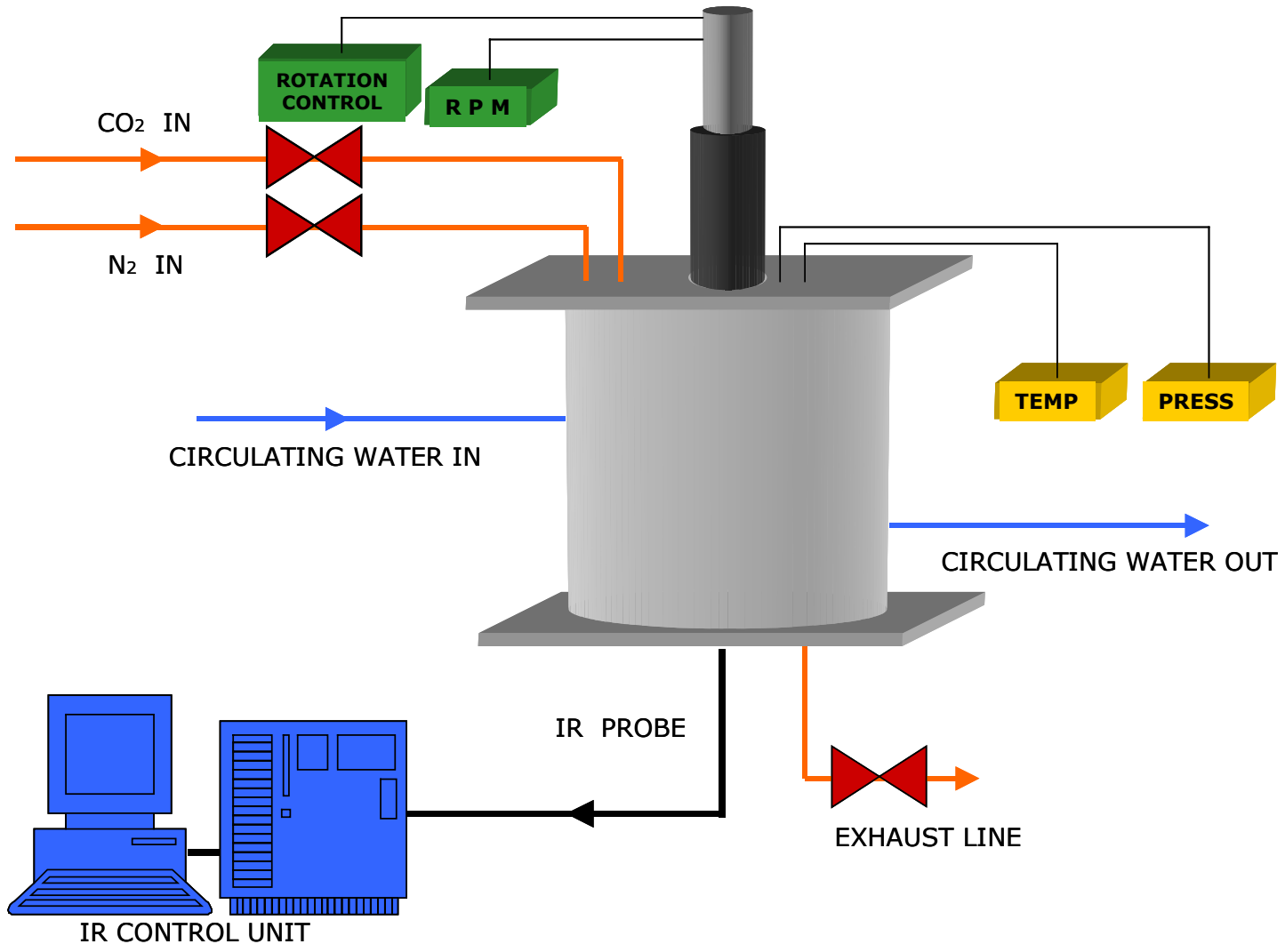

Figure 4.14. Schematic of the rotating disk dissolution monitoring apparatus. 
$\mathrm{CO}_{2}$

polymer flux $=k_{m}\left[C_{p}{ }^{*}-C_{p \infty}\right]$

penetrating into the film

polymer dissolving into fluid

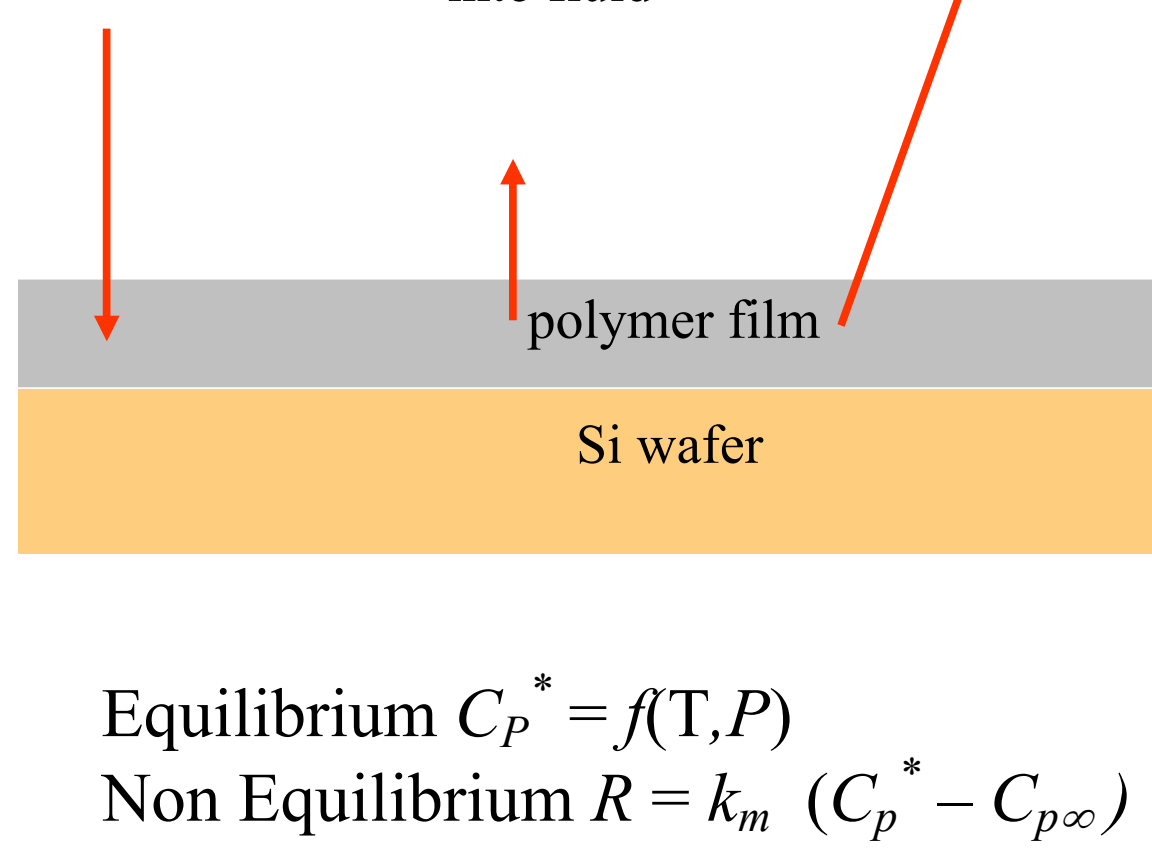

Figure 4.15. Relevant effects in the dissolution rate monitoring experiments. 
a)

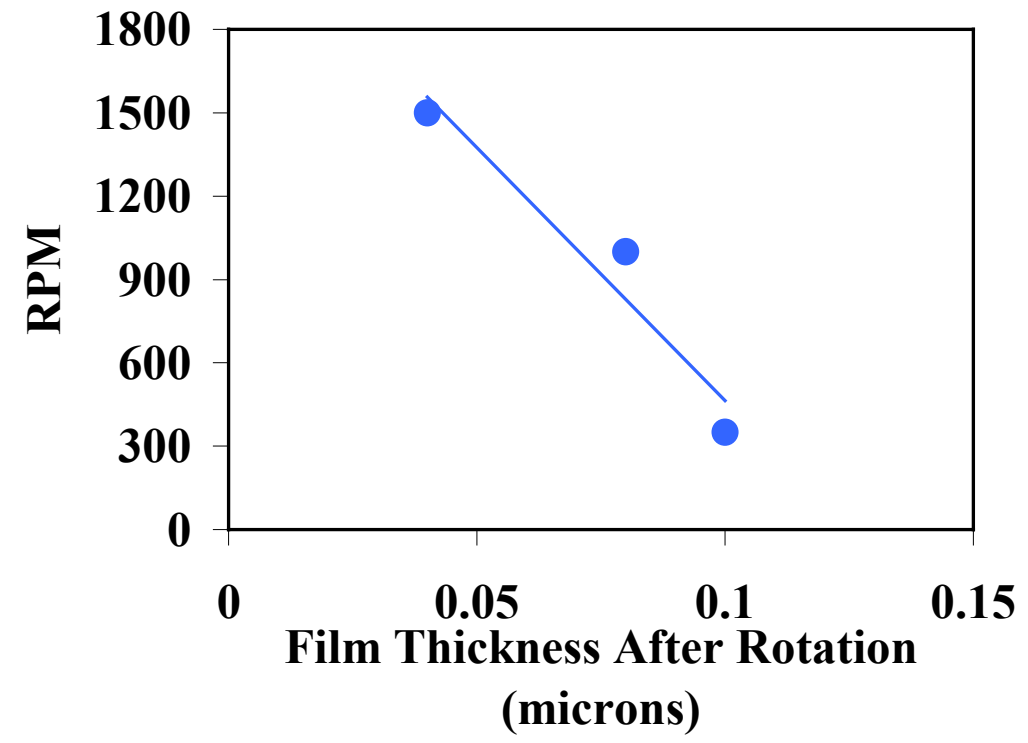

b)

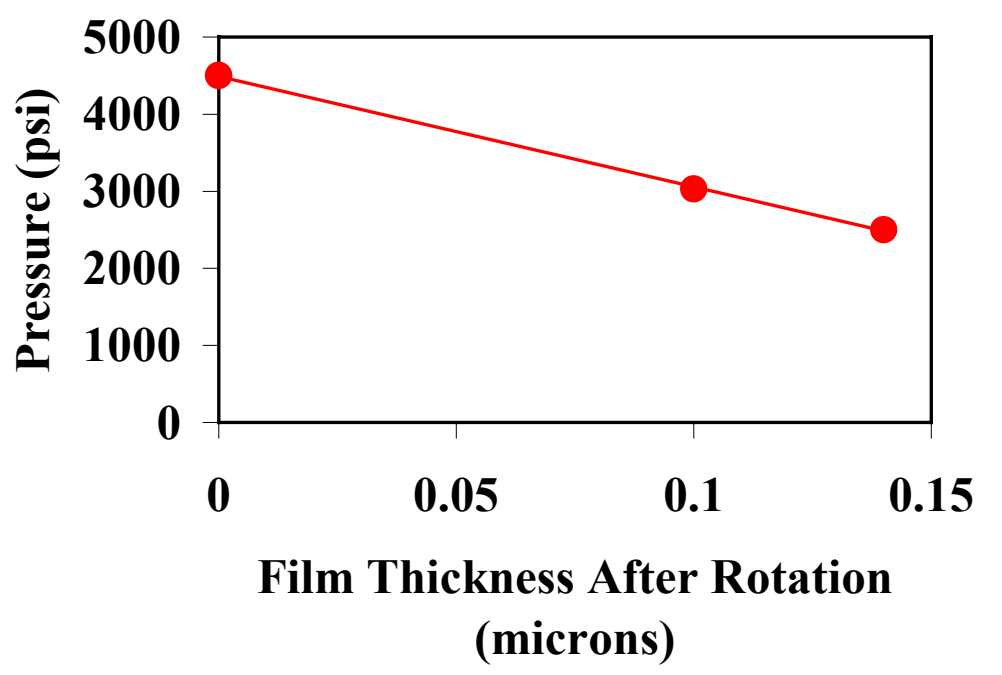

Figure 4.16. a) Remaining film thickness as a function of rotational speed; b) removed film thickness as a function of operating pressure. Copolymer resin used was PFOMA-rTHPMA 25k MW. 


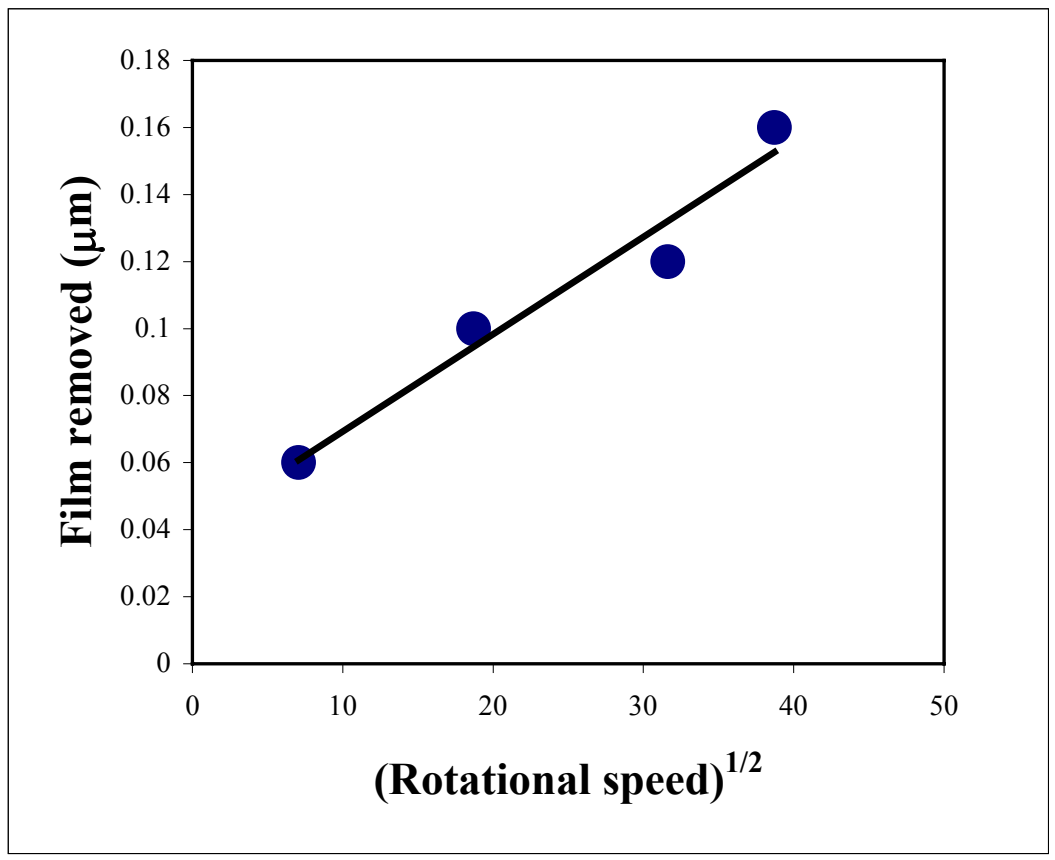

Figure 4.17. Portion of film removed plotted versus the square root of rotational speed. The linear data indicate that the process is diffusion limited. 
Chapter 5. $193 \mathrm{~nm}$ Lithography in $\mathrm{CO}_{2}$

\section{“Dry" Lithography Using Carbon Dioxide}

Erik N. Hoggan, Devin Flowers, Ruben G. Carbonell, Joseph M. DeSimone, to be submitted to Science, 2002. 


\begin{abstract}
Novel carbon dioxide $\left(\mathrm{CO}_{2}\right)$ soluble photoresists were synthesized based on random copolymers of poly(1,1-dihydroperfluorooctyl)methacrylate 2-tetrahyrdopyranyl methacrylate. These resins, along with suitable $\mathrm{CO}_{2}$-soluble photoacid generators, were utilized in a new "dry" lithographic process using only $\mathrm{CO}_{2}$ as a solvent. Photoresist spin casting, development, and stripping were all carried out in liquid and supercritical $\mathrm{CO}_{2}$. Parameters such as resist sensitivity, contrast, and resolution were investigated. Wafers were imaged using both $248 \mathrm{~nm}$ and $193 \mathrm{~nm}$ radiation, demonstrating the vast potential of this new photoresist platform.
\end{abstract}


The semiconductor industry is one of constant innovation, and has continued to keep pace with Moore's law of exponential progress for decades [1]. However, while feature sizes have consistently shrunk, and many of the processing steps have radically changed, key parts of the photolithographic process have remained static. For decades, photolithography has utilized a variety of organic and aqueous solvents to spin, develop, and strip photoresists on silicon wafers. In current commercial photolithography, which utilizes chemically amplified resists, spin casting and stripping are performed using various organic solvents, while resist development uses aqueous basic solutions such as tetramethyl ammonium hydroxide (TMAH) [2]. Although these processes have served the industry well for years, as feature sizes continue to shrink and exposure wavelengths drop to $157 \mathrm{~nm}$ and below, there are compelling reasons to reevaluate the use of these conventional solvents and implement a new technology.

Carbon dioxide, $\mathrm{CO}_{2}$, is well suited to replace conventional spin coating, developing, and stripping solvents in photolithography, yielding a completely integrated, "dry" solvent wafer processing. At first glance, the shift from a wet solvent-based system to one utilizing a compressed phase solvent such as $\mathrm{CO}_{2}$ may seem daunting. However, the potential benefits are numerous, and in recent years many $\mathrm{CO}_{2}$ based processes have proven their worth in other industries. $\mathrm{CO}_{2}$ has proven its value as an alternative solvent for a variety of industrial extraction processes,[3] polymer processing and synthesis[4] including $\mathrm{CO}_{2}$ based Teflon ${ }^{\circledR}$ production by Dupont, and even consumer $\mathrm{CO}_{2}$-based garment cleaning [5]. 
While any lithographic process utilizing $\mathrm{CO}_{2}$ as a solvent must necessarily operate at high pressure, there are many potential advantages of using $\mathrm{CO}_{2}$. The unique properties of $\mathrm{CO}_{2}$ allow it to address not one, but several of the challenges facing lithography in the near future. For instance, as exposure wavelengths decrease to $157 \mathrm{~nm}$ and below, many current photoresists become unusable due to their high optical density $(>5 / \mu \mathrm{m})$ at these wavelengths[6]. Thus most materials are ill-suited as $157 \mathrm{~nm}$ resists because the radiation cannot penetrate through films of the required thickness. However, Kunz et al. [7] have found that while most polymeric materials have a high optical absorbance at $157 \mathrm{~nm}$, fluoropolymers, hydrofluoropolymers and siloxane polymers are relatively transparent[8]. Thus the future generation of $157 \mathrm{~nm}$ resists are being developed utilizing this chemistry. There exists a large body of research in recent years showing that these same polymers often have excellent solubility in $\mathrm{CO}_{2}$ [9], [10]. In fact, when such polymers are specifically synthesized with $\mathrm{CO}_{2}$ solubility in mind, they are soluble under liquid $\mathrm{CO}_{2}$ conditions at concentrations as high as $30 \mathrm{wt} \%$, allowing one to form coatings using liquid $\mathrm{CO}_{2}$. And despite $\mathrm{CO}_{2}$ 's relatively high density in its compressed state $\left(711 \mathrm{~kg} / \mathrm{m}^{3}\right.$ at $25^{\circ} \mathrm{C}$ and vapor pressure), it maintains a low viscosity (57 $\mu \mathrm{Pa} \cdot \mathrm{s}$ at $25^{\circ} \mathrm{C}$ and vapor pressure compared with $1000 \mu \mathrm{Pa} \cdot \mathrm{s}$ for water at $\left.25^{\circ} \mathrm{C}\right)$ [11]. This low viscosity may be of great benefit in forming very thin and uniform films as wafer sizes increase to $300 \mathrm{~mm}$ and beyond.

Besides the issues with optical absorbance, the transition to $157 \mathrm{~nm}$ resists has introduced another significant problem, that of image collapse. As feature widths shrink below $90 \mathrm{~nm}$, while at the same time resist film thicknesses remain relatively large, very 
high aspect ratio features $(>10)$ are formed. When developing these features with conventional TMAH solutions, extreme forces are exerted on these small features due to the surface tension of the evaporating fluid. These surface tension forces often lead to feature collapse, destroying the desired image[12]. However, $\mathrm{CO}_{2}$ possesses a much smaller surface tension than water and other solvents [13]. Indeed, as pressure is increased, the density of the liquid and vapor become identical and at the critical point $\mathrm{CO}_{2}$ has zero surface tension. Namatsu, et al. have shown that it is possible to use supercritical $\mathrm{CO}_{2}$ to displace the aqueous developing fluid, and eliminate feature collapse even for very high aspect ratio features [14].

Not to be overlooked are the great environmental benefits of using $\mathrm{CO}_{2}$ in lithography. Indeed, these benefits are often the driving force behind the introduction of $\mathrm{CO}_{2}$ into many processes. Unlike the organic solvents and aqueous basic solutions used in conventional photolithography, $\mathrm{CO}_{2}$ is neither harmful to the environment, nor to persons working in close contact with it. And as each wafer must undergo the lithographic process 25 or more times to build up the requisite layers of circuitry and interconnects, large quantities of waste are generated in conventional lithography. Semiconductor fabrication facilities often consume enormous quantities of water per day (> 2000 gallons/day) [15]. The 1997 Semiconductor Industry Association (SIA) National Technology Roadmap for Semiconductors (NTRS) (and its 1999 update) and the 1996 Electronics Industry Environmental Roadmap (EEIR) both emphasize the importance of reducing wet organic and aqueous consumption (and in turn waste streams) in the industry. The complete replacement of the conventional solvents with $\mathrm{CO}_{2}$ would 
obviously meet this goal. And since $\mathrm{CO}_{2}$ is a byproduct of many other industrial reactions, and is also a recyclable material, no additional $\mathrm{CO}_{2}$ would be released into the atmosphere [16].

With these and other benefits in mind, we set about developing an all $\mathrm{CO}_{2}$ based photolithographic technology. Any such effort must of course begin with photoresists that are soluble in $\mathrm{CO}_{2}$. In our current work we utilize a random copolymer of poly $(1,1-$ dihydroperfluorooctyl)methacrylate 2-tetrahyrdopyranyl methacrylate (70:30, FOMA:THPMA). In a conventional organic solvent this copolymer would act as a positive resist, with the MAA acid being much more soluble than the original THPMA in a solution of TMAH. However, in $\mathrm{CO}_{2}$ the transformation from the poly(FOMA-rTHPMA) to a poly(FOMA-r-MAA) results in a less $\mathrm{CO}_{2}$-soluble material (Figure 1). This trend holds for many resist materials in $\mathrm{CO}_{2}$; polymers that act as a negative tone resist in conventional organic solvents will behave as positive tone resists in $\mathrm{CO}_{2}$. The resin synthesized in this work was found to be soluble in $\mathrm{CO}_{2}$ at moderate conditions (650 psi, $10^{\circ} \mathrm{C}$ ), and similar resins have been shown to be developable in $\mathrm{CO}_{2}$ by Ober et al. [17],[18]. However, as the goal was to perform completely dry lithography, not just development, it was necessary for us to spin coat the resin from liquid, not supercritical, $\mathrm{CO}_{2}$. In order to spin coat films of photoresist in $\mathrm{CO}_{2}$ one must have a liquid photoresist solution which evaporates into a controlled $\mathrm{CO}_{2}$ atmosphere. Thus, in order to achieve the correct solubility, all spin coating was performed under sub-ambient temperatures (6$\left.10{ }^{\circ} \mathrm{C}\right)[19]$. This small reduction in temperature increased the density and solvating strength of $\mathrm{CO}_{2}$ nearly $40 \%$, illustrating the extreme tunability of $\mathrm{CO}_{2}$ as a solvent . This 
change in $\mathrm{CO}_{2}$ solvent strength with temperature and pressure is what makes an all $\mathrm{CO}_{2}$ based lithography possible.

This principle is further illustrated in Figure 2, which shows how the photoresist can be spun, developed and stripped, using only $\mathrm{CO}_{2}$. By changing the temperature and pressure of the $\mathrm{CO}_{2}$, we are able to greatly modify its solvent strength. $\mathrm{CO}_{2}$ at moderate pressures and low temperatures will have a high density, and is suitable for spin coating. After exposure, the wafer may then be immersed in $\mathrm{CO}_{2}$ at a higher pressure and temperature, such that the unexposed photoresist will dissolve, but the exposed portions remain. Finally, after etching, the wafer may be immersed in $\mathrm{CO}_{2}$ again, but at a yet higher $\mathrm{CO}_{2}$ density, to remove the residual photoresist. Thus we are able to achieve three very different solvent strengths, and perform all steps of lithography using the single solvent, $\mathrm{CO}_{2}$.

The photoacid generators (PAGs) used were analogs to conventional PAGs but were highly fluorinated to provide suitable solubility in liquid $\mathrm{CO}_{2}$. Two variants were used, one ionic PAG and one nonionic PAG. The structure of each is given in Figure 1.

Polymer solutions for spin casting were prepared by dissolving $20 \mathrm{wt} \%$ of PFOMA-r-THPMA in liquid $\mathrm{CO}_{2}$ [20]. To achieve the necessary solvent strength, the $\mathrm{CO}_{2}$ was maintained at vapor pressure, but cooled to $6-10^{\circ} \mathrm{C}[21] . \mathrm{CO}_{2}$ soluble photoacid generator (PAG) was added in concentrations of $1-5 \mathrm{wt} \%$ with respect to the polymer concentration. This solution was maintained in a high-pressure cell with sapphire windows so that complete dissolution could be confirmed visually. The spin coating was carried out in a large high-pressure chamber, which could be loaded with $125 \mathrm{~mm}$ silicon 
wafers. Silicon wafers were placed on a rotating chuck with a drive shaft extending through the vessel and sealed with a custom rotating seal. Wafers were fixed to the chuck via the pressure differential between the chamber and the atmosphere, operating on a principle similar to a conventional vacuum chuck, but utilizing high-pressure rather than a vacuum. The spinning chamber was charged with $\mathrm{CO}_{2}$ vapor. The pressure in the spinning chamber was maintained at precise values ranging from 5-15 psi below the equilibrium vapor pressure of the $\mathrm{CO}_{2}$-photoresist solution [22]. Control of this chamber pressure was a key parameter in the determination of film quality and thickness, as the pressure differential was what governed the evaporation rate of the liquid $\mathrm{CO}_{2}$. Precision control of the evaporation rate, spinning speed, and system temperature were required to ensure consistent, uniform coatings [23]. After spinning, the $\mathrm{CO}_{2}$ was vented from the chamber and the wafer was then removed for exposure. Because of the high volatility of $\mathrm{CO}_{2}$, wafers did not require the normal post apply bake used to drive off residual solvent in conventional spin casting. It should also be noted that because $\mathrm{CO}_{2}$ evaporates so readily from the film upon depressurization, that there is no out-gassing of residual spin casting solvent during subsequent steps, which is often a concern when using conventional solvents. Extensive work was conducted to determine the ideal range of rotational speeds, pressures, and concentrations [24] to be used to produce uniform, lithographic quality films, resulting in films with 3\% variation in film thickness over the entire wafer, and an r.m.s. roughness of 0.4-0.5 nm [25]. Films used in the imaging experiments were $350-500 \mathrm{~nm}$ in thickness, depending upon the conditions used. 
Initial exposures were performed on a deep-UV flood exposure tool (Oriel) with a $248 \mathrm{~nm}$ filter. To determine the optimum resist formulation and conditions, a series of photoresists were formulated as shown in Table 1. Exposed wafers were then sectioned into strips and developed in a $\mathrm{CO}_{2}$ flow-through chamber. The chamber and a $\mathrm{CO}_{2}$ filled syringe pump (Isco) were preheated to the appropriate temperature $\left(60^{\circ} \mathrm{C}\right)$. Development was then carried out by flowing the preheated, supercritical $\mathrm{CO}_{2}$ through the chamber at a constant flow rate, while maintaining constant pressure via computer controlled pneumatic valves (Badger Meter). Short development times on the order of 30-90 seconds were found to be sufficient.

After development, film thicknesses were characterized via profilometry (Tencor) and sensitivity curves were generated. The sensitivity curves were made by plotting the remaining film thicknesses after development versus the exposure dosage $\left(\mathrm{mJ} / \mathrm{cm}^{2}\right)$. The curve provides an understanding of how much dosage is required to render the desired portions of the film insoluble in $\mathrm{CO}_{2}$, and also shows the abruptness of the transition from soluble to insoluble polymer. To achieve well-defined, crisp features, a sharper transition (high contrast) is desired. Figure 3 shows two such curves, showing the performance of each PAG using both $248 \mathrm{~nm}$ and $193 \mathrm{~nm}$ radiation. In both instances, the contrast $(\gamma)$ achieved using the nonionic PAG is higher than that using the ionic PAG, however, the sensitivity is also higher than desired $\left(20-35 \mathrm{~mJ} / \mathrm{cm}^{2}\right.$ for the nonionic PAG as opposed to $5-19 \mathrm{~mJ} / \mathrm{cm}^{2}$ for the ionic PAG). This may be attributed in part to the lower absorption of the nonionic PAG at these wavelengths. However, both sensitivity curves indicated 
sufficient contrast between exposed and unexposed regions of the resist, making these resist formulations suitable for imaging.

Using these formulations, wafers were imaged using both 248 and $193 \mathrm{~nm}$ exposures. For the initial 248 exposures, contact printing using a mask with test feature widths ranging from $2 \mu \mathrm{m}$ to $10 \mu \mathrm{m}$ was carried out with a flood exposure tool. Further experiments were carried out at Clariant Corp. using a commercial stepper. Wafers were also imaged using $193 \mathrm{~nm}$ radiation. Although both the resin and PAGs are suitable for $193 \mathrm{~nm}$ lithography, high quality images could not be obtained with the 193 exposures within the limit of the reticle (patterns were $0.3 \mu \mathrm{m}$ or smaller). The same experiments were repeated using $248 \mathrm{~nm}$ radiation and a reticle with much larger feature sizes $(0.25$ $\mu \mathrm{m}$ to $2.5 \mu \mathrm{m}$ ). Scanning electron micrographs (SEMs) of the developed photoresist features can be seen in Figure 4. Using this system it was possible to resolve 1:1 lines and spaces down to widths of $0.8 \mu \mathrm{m}$. Features smaller than this value were of poor quality and were blurred due to merging of adjacent lines. One explanation for this limitation in feature size is due to the low glass transition temperature $\left(\mathrm{T}_{\mathrm{g}}\right)$ of the polymer resin $\left(49^{\circ} \mathrm{C}\right.$ for the $25 \mathrm{k}$ MW resin). In order to drive the cleavage of the leaving group, post exposure baking (PEB) temperatures of $90-105^{\circ} \mathrm{C}$ were used. Experiments carried out at PEBs of $50^{\circ} \mathrm{C}$ and $70{ }^{\circ} \mathrm{C}$ did not yield any images. However, even the lowest value of the PEB at which images could be resolved $\left(90^{\circ} \mathrm{C}\right)$ was nearly $40{ }^{\circ} \mathrm{C}$ above the $\mathrm{T}_{\mathrm{g}}$ of the polymer resin $\left(49^{\circ} \mathrm{C}\right)$. The increased polymer and acid mobility at these temperatures far above $\mathrm{T}_{\mathrm{g}}$ are the likely cause of poor resolution on the smallest feature 
sizes. Future work is focused on the replacement of current resins with more thermally stable materials.

It should be noted that these features were produced with very little optimization of the photoresist system. Variables such as the PEB temperature, PAG concentration, and development temperature all have ample room for adjustment. To achieve such promising results with so little optimization demonstrates the remarkable potential of $\mathrm{CO}_{2}$ based processing. With further refinements in both the photochemistry and the processing conditions, it may soon be possible to achieve performance equal to or greater than conventional solvent based lithography, using only liquid and supercritical $\mathrm{CO}_{2}$ as the solvent.

Acknowledgement. The authors thank Prof. Clifford L. Henderson and Cody Berger at the Georgia Institute of Technology for the use of his deep-UV exposure facilities. The authors also thank Ralph Dammel, Frank Houlihan, and Takanori Kudo at Clariant Corp. for $248 \mathrm{~nm}$ and $193 \mathrm{~nm}$ exposures. We thank the NSF Science and Technology Center for Environmentally Responsible Solvents and Processes. 


\section{References}

1. Risch, L., The end of the CMOS roadmap - new landscape beyond. Materials Science and Engineering C, 2002. 19: p. 363-368.

2. Levinson, H.J., Principles of Lithography. 2001, Bellinghman, Washington: SPIE Press.

3. Erkey, C., Supercritical carbon dioxide extractions of metals from aqueous solutions: a review. Journal of Supercritical Fluids, 2000. 17(3): p. 259-287.

4. Cooper, A.I., Polymer synthesis and processing using supercritical carbon dioxide. Journal of Materials Chemistry, 2000. 10(2): p. 207.

5. DeSimone, J.M., et al., Cleaning process using carbon dioxide as a solvent and employing molecularly engineered surfactants. 1999, The University of North Carolina at Chapel Hill: USA.

6. Bloomstein, T., et al., Critical issues in $157 \mathrm{~nm}$ lithography. Journal of Vacuum Science \& Technology B, 1998. 16(6): p. 3154-3157.

7. Kunz, R.R., et al., Outlook for $157 \mathrm{~nm}$ Resist Design. J. Vac. Sci. Technol. B, 1999. 17(6): p. 3267-3272.

8. Fluorinated norborne based resins that were synthesized in conjuction with this work have absorbances at $157 \mathrm{~nm}$ as low as $1.5 / \mu m$.

9. McHugh, M.A. and V.J. Krukonis, Supercritical Fluid Extraction: Principle and Practice. 2nd ed. 1994, Stoneham, MA: Butterworths. 
10. DeSimone, J.M., Z. Guan, and C.S. Eisbernd, Synthesis of Fluoropolymers in Supercritical Carbon Dioxide. Science, 1992. 257: p. 945-947.

11. Span, R. and W. Wagner, A new equation of state for carbon dioxide covering the fluid region from the triple-point temperature to $1100 \mathrm{~K}$ at pressures up to 800 MPa, in J. Phys. Chem. Ref. Data. 1996. p. 1509-1596.

12. Tanaka, T., M. Morigami, and N. Atoda, Mechanism of Resist Pattern Collapse during Development Process. J. Appl. Phys., 1993. 32(12B): p. 6059-6064.

13. The surface tension for liquid $\mathrm{CO}_{2}$ at $25^{\circ} \mathrm{C}$ is $6.74 \times 10^{-4} \mathrm{~N} / \mathrm{m}$, which is two orders of magnitude lower than water at $25^{\circ} \mathrm{C}(0.072 \mathrm{~N} / \mathrm{m})$. A typical organic such as acetone which has a surface tension of $0.024 \mathrm{~N} / \mathrm{m}$ at $25^{\circ} \mathrm{C}$.

14. Namatsu, H., K. Yamazaki, and K. Kurihara, Supercritical resist dryer. J. Vac. Sci. Technol. B, 2000. 18(2): p. 780-784.

15. Wetmore, P.M., Supercritical Fluid Technology for Photoresist Developing, in Chemical Engineering. 1997, University of Massachusetts: Lowell.

16. Hashimoto, K., et al., Advanced materials for global carbon dioxide recyling. Materials Science and Engineering A, 2001. 304: p. 88-96.

17. Sundararajan, N., et al., Supercritical $\mathrm{CO}_{2}$ processing for submicron imaging of fluoropolymers. Chemistry of Materials, 2000. 12(1): p. 41-49.

18. Ober, C.K., et al., Imaging Polymers with Supercritical Carbon Dioxide. Advanced Materials, 1997. 9(13): p. 1039-1043.

19. Solubility measurements were conducted using a small $10 \mathrm{ml}$ high-pressure view cell with sapphire windows. Experiments were conducted by fixing the cell 
temperature at the desired value and then slowly raising the pressure until the resin dissolved in the $\mathrm{CO}_{2}$. When the solution transitioned from opaque to transparent, dissolution was deemed to be complete. Using 1 wt\% samples, it was found that $5 \mathrm{~K} M W$ poly(FOMA-r-THPMA) was soluble at $25^{\circ} \mathrm{C}$ and $1350 \mathrm{psi}$, and at $10{ }^{\circ} \mathrm{C}$ at vapor pressure, $15 \mathrm{~K} M W$ poly(FOMA-r-THPMA) was soluble at $25^{\circ} \mathrm{C}$ and $1400 \mathrm{psi}$, and at $10^{\circ} \mathrm{C}$ at vapor pressure, and $25 \mathrm{KMW}$ poly(FOMA-rTHPMA) was soluble at $25^{\circ} \mathrm{C}$ and 1450 psi, and at $10^{\circ} \mathrm{C}$ at vapor pressure.

20. Solution concentration directly affects solution viscosity, which is a key parameter in film uniformity and thickness. Solution viscosities were measured for 5-20\% resin in $\mathrm{CO}_{2}$ using a falling cylinder viscometer. At a concentration of $20 w t \%$ resin, the viscosity was found to be $0.597 \mathrm{cP}$.

21. The vapor pressure of $\mathrm{CO}_{2}$ varies significantly with temperature. At a typical operating temperature of 8 degrees $C$, the vapor pressure of the $\mathrm{CO}_{2}$-photoresist solution was 625 psi..

22. Solution pressure was measured with a transducer accurate to 0.3 psi (Druck) and fed to a control program (Bridgeview) which compared the solution pressure to the chamber pressure and adjusted the pressure venting control (Badger Meter) accordingly. The total accuracy of the pressure control system was on the order of $+/-0.8$ psi.

23. Films used in this study were spun with a pressure differential of 10 psi at 2000 RPM and 8 degrees $C$. Fill times for the spinning chamber were on the order of 5 minutes. Films were spun for 60 seconds and then the chamber was evacuated 
over 6 minutes. A second generation device currently being constructed should greatly reduce fill and venting times.

24. Spin coating trials were conducted at rotational speeds ranging from $750 \mathrm{rpm}$ to $2500 \mathrm{rpm}$, concentrations from $5 \mathrm{wt} \%$ to $25 \mathrm{wt} \%$ resin in $\mathrm{CO}_{2}$, and evaporative driving forces $(\Delta P)$ of 0 to 25 psi. The highest film quality was obtained with $\Delta P$ values of 10-15 psi, and concentrations of $15 \mathrm{wt} \%$ or larger.

25. Roughness results were obtained via atomic force microscopy by Dario Goldfarb, IBM Corporation, Yorktown Heights, NY. 
a)<smiles>c1ccc([I-]c2ccccc2)cc1</smiles>

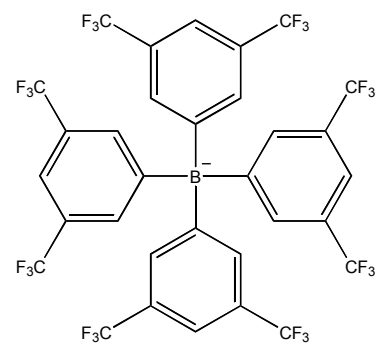

b)

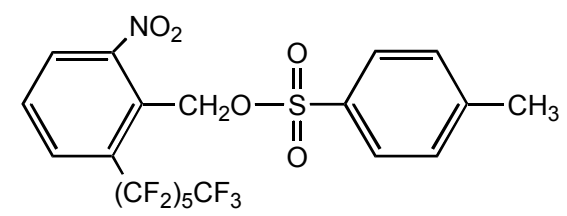

c)

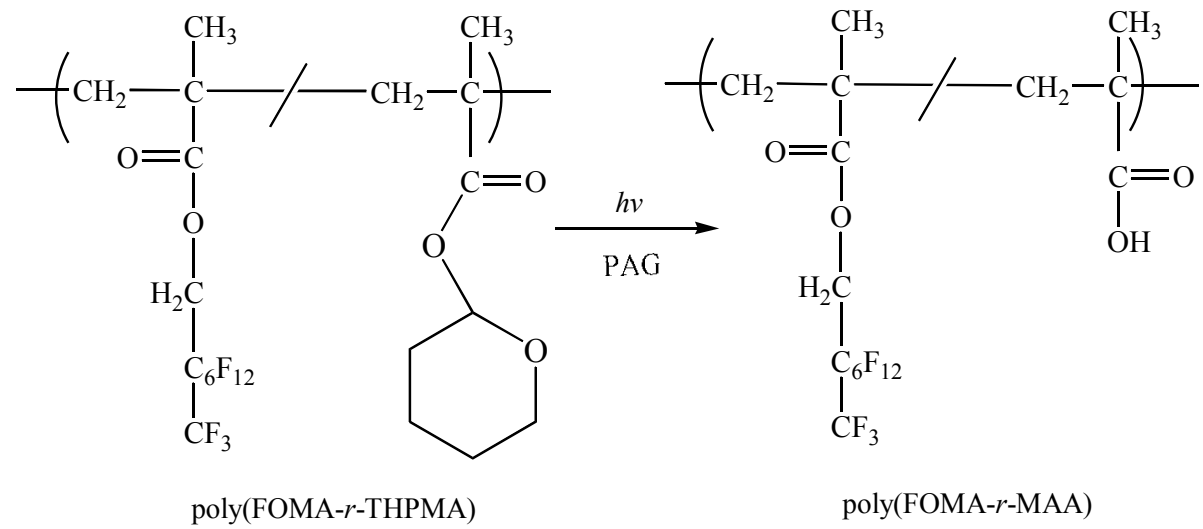

Figure 5.1. Structure of $\mathrm{CO}_{2}$ soluble photoresist system: a) ionic PAG, Diphenyliodonium tert-(3,5-bis(trifluoromethyl) phenyl boronium. Diphenyliodonium tert-[3,5bis(trifluoromethyl) phenyl] borate was prepared by an ion-exchange reaction between diphenyliodonium chloride and sodium tert-[3,5-bis(trifluoromethyl) phenyl] borate $\left(3 \mathrm{H}_{2} \mathrm{O}\right)$ in methylene chloride at room temperature for 24 hours b) nonionic PAG, 2perfluorohexyl-6-nitrobenzyl tosylate. 2-Perfluorohexyl-6-nitrobenzyl tosylate was synthesized in a three step process. First, perfluorohexyl iodide and 2-bromo-6nitrotoluene was reacted in a cross-coupling with copper bronze at $125{ }^{\circ} \mathrm{C}$ for $48 \mathrm{~h}$ in dimethyl sulfoxide (DMSO) under $\mathrm{N}_{2}$ to give 2-Methyl-3-nitrobenzoperhexyl fluoride. The methyl group was brominated by heating a reaction mixture of benzyl peroxide (BPO) and N-bromosuccinimide (NBS) to $70{ }^{\circ} \mathrm{C}$ in carbon tetrachloride $\left(\mathrm{CCl}_{4}\right)$ for $72 \mathrm{~h}$ while irradiating with $\mathrm{Hg}$ lamp. Finally, the benzyl bromide was substituted using silver tosylate in dry acetonitrile at $70{ }^{\circ} \mathrm{C}$ for $24 \mathrm{~h}$ under $\mathrm{N}_{2}$. c) $\mathrm{CO}_{2}$ soluble resin, poly $(1,1-$ dihydroperfluorooctyl)methacrylate 2-tetrahyrdopyranyl methacrylate. Poly(FOMA-rTHPMA) was prepared free-radically with Vazo ${ }^{\circledR} 52$ in $\alpha$-trifluorotoluene while stirring under $\mathrm{N}_{2}$ at $60{ }^{\circ} \mathrm{C}$ for 23 hours. 

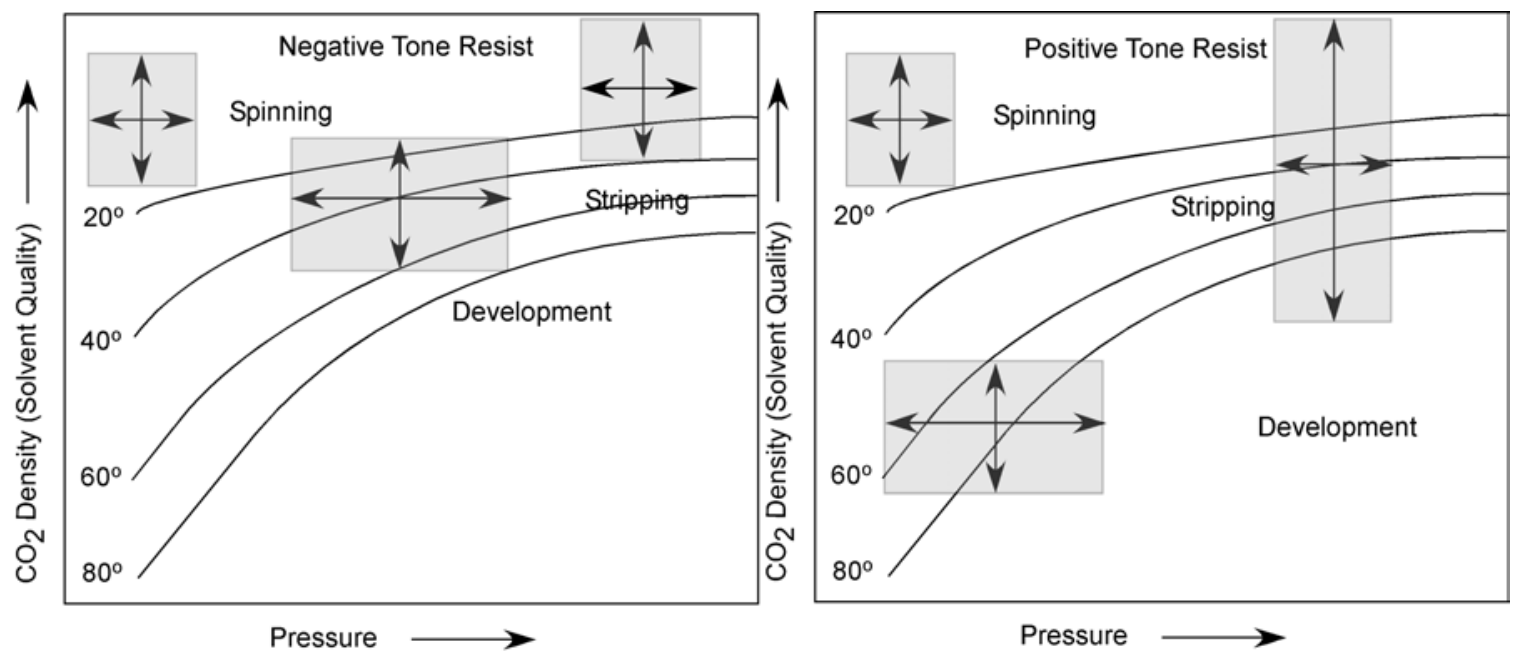

Figure 5.2. Schematic of how $\mathrm{CO}_{2}$ may be used for spinning, developing and stripping by altering the solvent strength for each step. Although not exact, solvent strength is approximated by $\mathrm{CO}_{2}$ density. The grey area indicates the development window, or the range of temperatures and pressures in which the $\mathrm{CO}_{2}$ has sufficient solvent strength to remove parts of the photoresist, but is mild enough to leave the desired portions intact. If the solvent strength is increased into the stripping regime, the remaining resist is removed. 

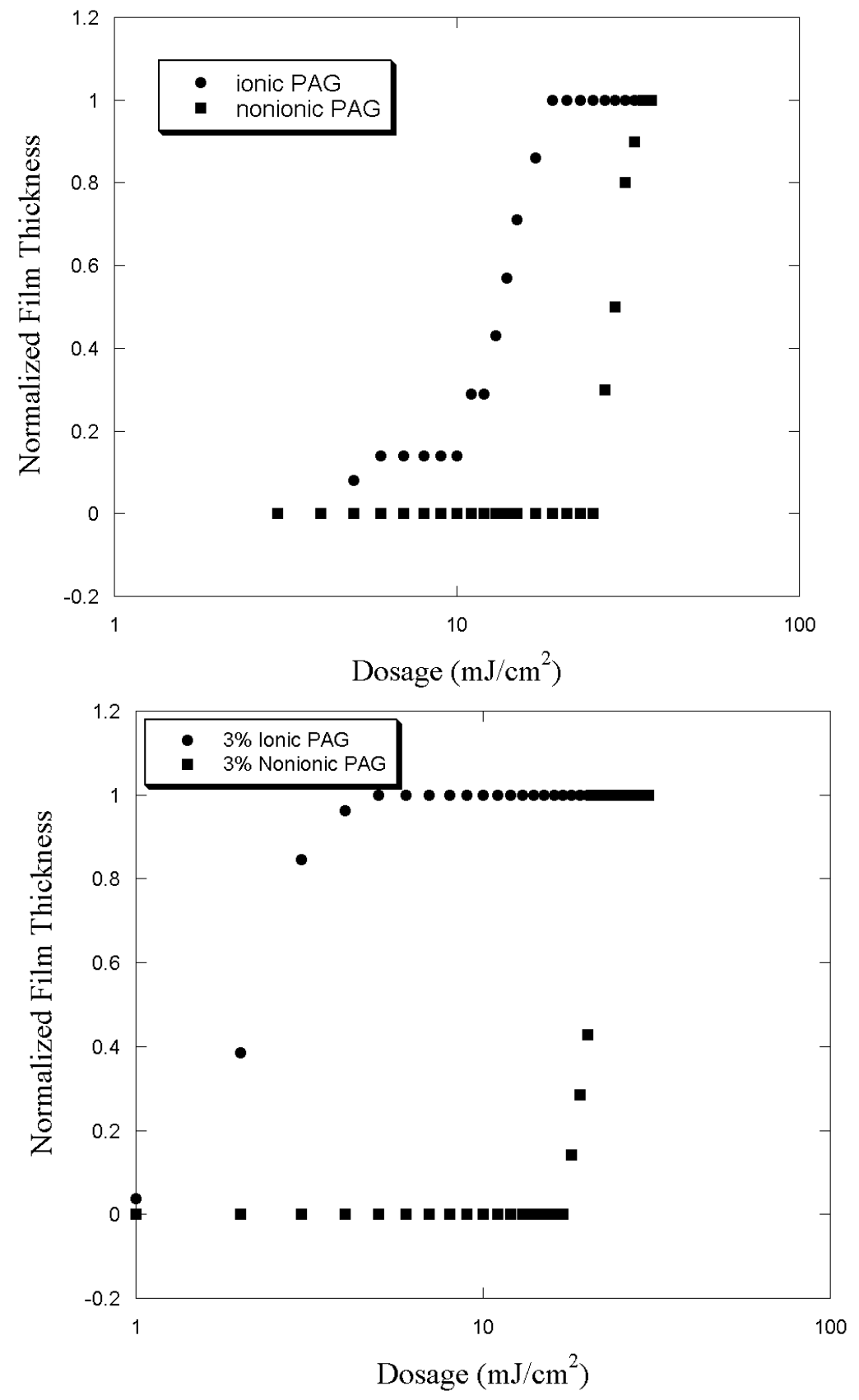

Figure 5.3. a) Sensitivity plot for $15 \mathrm{k}$ MW PFOMA- $r$-THPMA with both ionic and nonionic PAGs, PEB $105^{\circ} \mathrm{C}$, developed in $\mathrm{scCO}_{2}$ at $4400 \mathrm{psi}, 60^{\circ} \mathrm{C}$, exposed at $248 \mathrm{~nm}$. b) Sensitivity plot for $25 \mathrm{k}$ MW PFOMA- $r$-THPMA with both ionic and nonionic PAGs, PEB $90{ }^{\circ} \mathrm{C}$, developed in $\mathrm{scCO}_{2}$ at $4200 \mathrm{psi}, 60^{\circ} \mathrm{C}$, exposed at $193 \mathrm{~nm}$. Film thicknesses were measured via profilometry (Tencor). 

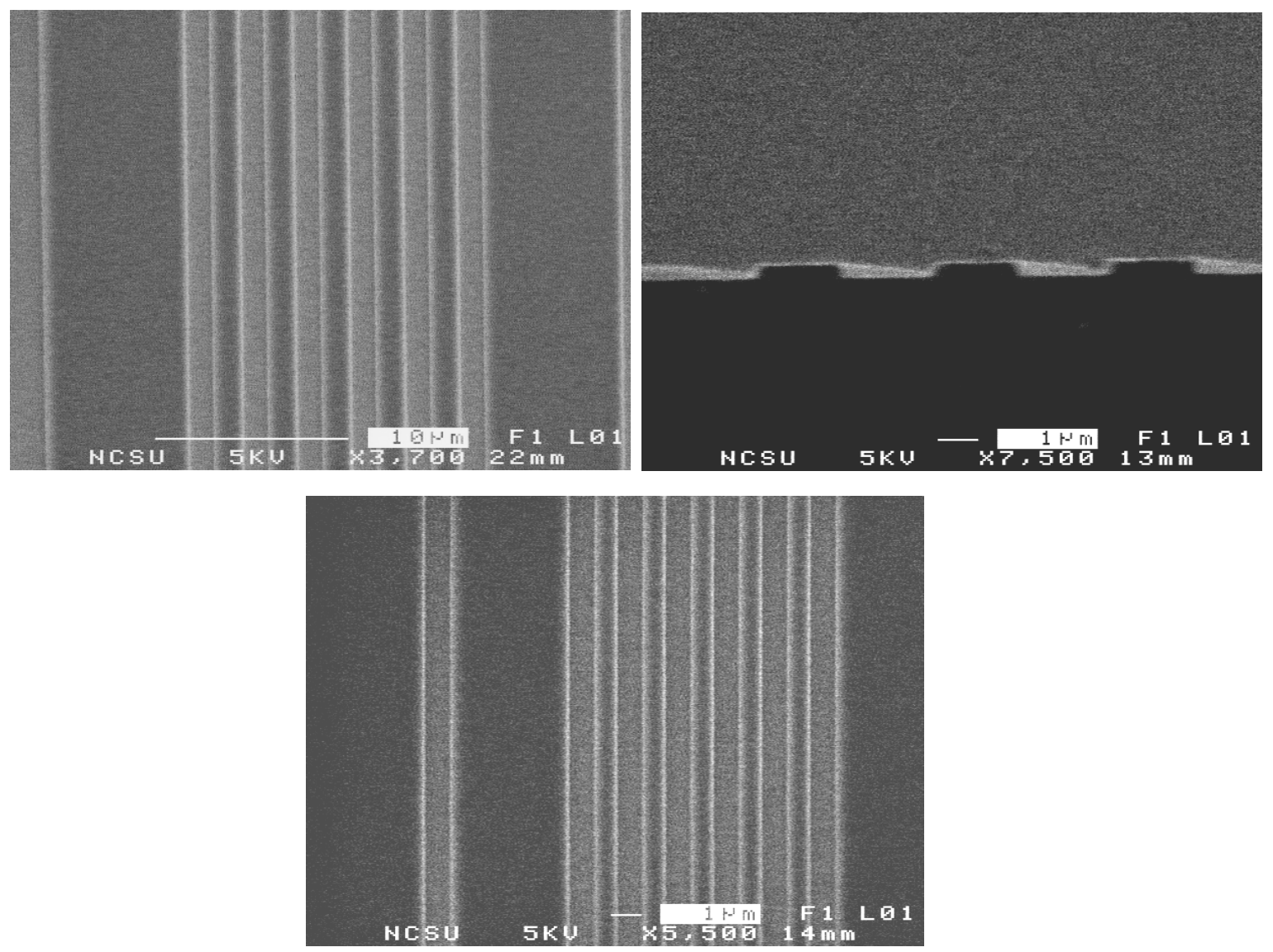

Figure 5.4. $248 \mathrm{~nm}$ exposures of $25 \mathrm{k}$ MW Poly(FOMA-r-THPMA) photoresist with 5\% ionic PAG, $105^{\circ} \mathrm{C}$ PEB, developed in supercritical $\mathrm{CO}_{2}$ at $4200 \mathrm{psi}, 60^{\circ} \mathrm{C}$ for 45 seconds. Images were taken using a JEOL 6400F field emission SEM. 
Table 5.1. Resins and PAGs investigated. All resins were poly(FOMA-r-THPMA) with target molecular weights as indicated.

\begin{tabular}{|c|c|c|c|c|c|c|}
\hline \multicolumn{7}{|c|}{$248 \mathrm{~nm}$ exposures } \\
\hline $\begin{array}{c}\text { Resin Molecular } \\
\text { Weight }\end{array}$ & $P A G$ & $\begin{array}{c}P A G \\
\text { loading }\end{array}$ & $\begin{array}{l}\text { PEB } \\
\left({ }^{\circ} \mathrm{C}\right)\end{array}$ & $\begin{array}{l}\text { Developing } \\
\text { Conditions }\end{array}$ & Contrast & $\begin{array}{l}\text { Sensitivity } \\
\left(\mathrm{mJ} / \mathrm{cm}^{2}\right)\end{array}$ \\
\hline $15 \mathrm{k}$ & ionic & $5 \%$ & 90 & $4100 \mathrm{psi} / 60^{\circ} \mathrm{C}$ & 3.6 & 19 \\
\hline $15 \mathrm{k}$ & nonionic & $5 \%$ & 105 & $4100 \mathrm{psi} / 60^{\circ} \mathrm{C}$ & 6.8 & 35 \\
\hline $25 \mathrm{k}$ & ionic & $5 \%$ & 90 & $4400 \mathrm{psi} / 60^{\circ} \mathrm{C}$ & 3.3 & 12 \\
\hline $25 \mathrm{k}$ & ionic & $5 \%$ & 105 & $4400 \mathrm{psi} / 60^{\circ} \mathrm{C}$ & 2.6 & 17 \\
\hline $25 \mathrm{k}$ & ionic & $5 \%$ & 120 & $4400 \mathrm{psi} / 60^{\circ} \mathrm{C}$ & 2.4 & 19 \\
\hline $35 \mathrm{k}$ & ionic & $5 \%$ & 105 & $4400 \mathrm{psi} / 60^{\circ} \mathrm{C}$ & 1.2 & 19 \\
\hline $35 \mathrm{k}$ & nonionic & $5 \%$ & 105 & $4400 \mathrm{psi} / 60^{\circ} \mathrm{C}$ & 4.1 & 35 \\
\hline \multicolumn{7}{|c|}{$193 \mathrm{~nm}$ exposures } \\
\hline $25 \mathrm{k}$ & ionic & $3 \%$ & 90 & $4300 \mathrm{psi} / 60^{\circ} \mathrm{C}$ & 1.4 & 5 \\
\hline $25 \mathrm{k}$ & nonionic & $3 \%$ & 90 & $4300 \mathrm{psi} / 60^{\circ} \mathrm{C}$ & 10.8 & 21 \\
\hline
\end{tabular}




\section{Chapter 6. Conclusions and Future Directions}

This work has demonstrated that it is indeed possible to spin coat photoresist films using only liquid $\mathrm{CO}_{2}$ as the solvent. The resultant films are of sufficient quality to be used in photolithography, although further improvements are certainly necessary. The $\mathrm{CO}_{2}$ spin coating process behaves as theory predicts. This agreement between theory and experimental results is significant, not only because it confirm that our fundamental understanding of the process is correct, but also in that it allows us to extend our experience to other polymer systems to achieve films of the desired thickness without the extensive trial-and-error inherent in the early work.

We have shown that the $\mathrm{CO}_{2}$ photoresists have sensitivities and contrasts competitive with current commercial resists. This thesis has detailed the exposure, characterization, and patterning of these resists at both $248 \mathrm{~nm}$ and $193 \mathrm{~nm}$. While feature sizes are not yet comparable to conventional resists, results are very promising and future improvements should be significant. Much of this work was done in highpressure laboratories, well suited to $\mathrm{CO}_{2}$, but with lithographic equipment primitive by current standards. Further experiments on state-of-the-art photolithographic tools will no doubt produce far superior results.

\section{Future Directions}

One important area of future research is the improvement of the development processes used. Although it was sufficient for this work, the current $\mathrm{CO}_{2}$ developer is a relatively simple design with room for several improvements. Of paramount importance 
to this effort is ongoing work stemming from the collaboration between the author and Mohamad Elbaccouch in the modeling and measurement of the dissolution kinetics of photoresist films on silicon wafers.

As the $\mathrm{CO}_{2}$ spin coating effort proceeds, it is necessary to progress beyond the small test wafers used in this work and demonstrate the feasibility of this technology on 8 " wafers. No theoeretical barriers exist to this transition, but there will no doubt be process and engineering challenges associated with the increase in wafer size. This change is already well under way. An 8" spin coater is currently being assembled to complete these experiments. The main pressure vessel for this coater was manufactured by Uhde, and contains many improvements over the apparatus used in this work. Further details are given in Appendix B.

Another issue that warrant further investigation is the actual performance of these materials as photoresists. A precise evaluation of the adhesion, durability, and etch resistance of these materials was beyond the scope of this work. Some brief experiments etch resistance experiments were described earlier, but a far more extensive study will be required before the transition to industrial fabrication can proceed.

Much work remains to be done, but this thesis has described huge leaps in our understanding of $\mathrm{CO}_{2}$ based photolithography. If future work builds upon these advances as expected, it will soon be possible to replace conventional solvents throughout the photolithographic process, resulting in a high performance, environmentally benign dry lithography. 


\section{Appendix A. Analysis of Spin Coating in Liquid $\mathrm{CO}_{2}$}

As mentioned in the introduction, in nearly all conventional spin coating processes, solvent evaporates throughout the spinning and the final dry film is formed prior to deceleration. However, such a process has proved difficult to control in $\mathrm{CO}_{2}$ spin coating and the current process involves the creation of a thin liquid film which then must be purged of $\mathrm{CO}_{2}$ in a separate step. An analysis of this process closely follows the pioneering work of Emslie, Bonner, and Peck [1].

\section{General Solution}

Assumptions:

1. There is no evaporation.

2. The rotating disk is infinite.

3. The plane is horizontal, so there is no radial gravitational component.

4. The liquid layer is radially symmetric and sufficiently thin that gravitational potential normal to the surface has negligible effect on distributing the liquid.

5. The fluid is Newtonian.

6. The liquid layer is sufficiently thin that shear resistance is only appreciable in the horizontal direction.

The spinning disk is analyzed in cylindrical coordinates $(\mathrm{r}, \theta, \mathrm{z})$ with the disk spinning at an angular velocity of $\Omega$. The $\mathrm{z}$ dependence of the radial velocity of the liquid, $\mathrm{v}_{\mathrm{r}}$, is found by applying the above assumptions to the Cauchy equation of motion, yielding 
$-\eta \frac{\partial v_{r}}{\partial z^{2}}=\rho \Omega^{2} r$

where $\eta$ is the viscosity and $\rho$ is the density of the liquid. The two boundary conditions needed are: no slip at the substrate surface and equal shear stresses at the liquid-vapor interface,

$$
\begin{aligned}
& v_{r}=0 @ H=0 \\
& \frac{\partial v_{r}}{\partial z}=0 @ z=H .
\end{aligned}
$$

Integrating with respect to $\mathrm{z}$ yields

$$
\frac{\partial v_{r}}{\partial z}=-\frac{\rho \Omega^{2} r z}{\eta}+C_{1} .
$$

By applying the first boundary condition, we find

$$
C_{1}=\frac{\rho \Omega^{2} r H}{\eta} .
$$

Substituting and integrating again gives the expression,

$v_{r}=-\frac{\rho \Omega^{2} r z^{2}}{2 \eta}+\frac{\rho \Omega^{2} r H z}{\eta}+C_{2}$.

Using the last boundary condition, we find $\mathrm{C}_{2}=0$. Thus, the expression for the radial velocity of the liquid is

$v_{r}=\frac{1}{\eta}\left(-\frac{1}{2} \rho \Omega^{2} r z^{2}+\rho \Omega^{2} r z\right)$. 
To relate the radial velocity to the overall height of the film, it is necessary to recast the problem in terms of radial flow. The radial flow per unit length of circumference, $q$, is

$$
q=\int_{0}^{h} v_{r} d z=\frac{\rho \Omega^{2} r H^{3}}{3 \eta} .
$$

To obtain an expression for the change in film height with time, we apply the mass continuity equation,

$\frac{1}{\mathrm{r}} \frac{\partial}{\partial \mathrm{r}}\left(\mathrm{rv}_{\mathrm{r}}\right)+\frac{\partial}{\partial \mathrm{z}}\left(\mathrm{v}_{\mathrm{z}}\right)=0$,

where $\mathrm{v}_{\mathrm{Z}}$ is the fluid velocity in the vertical direction. Substituting for $\mathrm{v}_{\mathrm{r}}$ and rearranging yields

$$
\frac{1}{\mathrm{r}} \frac{\partial}{\partial \mathrm{r}}\left(\mathrm{r} \frac{\partial \mathrm{q}}{\partial \mathrm{z}}\right)=-\frac{\partial}{\partial \mathrm{z}}\left(\mathrm{v}_{\mathrm{z}}\right) .
$$

Applying the product rule leads to

$$
\frac{1}{r}\left(r \frac{\partial q}{\partial r \partial z}+\frac{\partial q}{\partial z}\right)=-\frac{\partial}{\partial z}\left(v_{z}\right) \text {. }
$$

We integrate with respect to $z$ to obtain

$$
\frac{1}{r}\left(r \frac{\partial q}{\partial r}+q\right)=-v_{z} \text {. }
$$

Recognizing that $\mathrm{v}_{\mathrm{z}}=\partial \mathrm{H} / \partial \mathrm{t}$ and simplifying the left hand side gives

$$
\frac{\partial H}{\partial t}=-\frac{1}{r} \frac{\partial(r q)}{\partial r} .
$$


Substituting in the expression for $\mathrm{q}$ and differentiating results in

$$
\frac{\partial H}{\partial t}=-\frac{\rho \Omega^{2}}{3 \eta} \frac{1}{r} \frac{\partial}{\partial r}\left(r^{2} H^{3}\right) .
$$

Special Solution for the Case of an Initially Uniform Distribution

Assuming that the initial film thickness is thick but approximately uniform, the film height will depend only on time. Equation A.12 then reduces to,

$$
\frac{d H}{d t}=-2 \frac{\rho \Omega^{2}}{3 \eta} H^{3} .
$$

Integrating this equation leads to the following expression for $\mathrm{h}$ as a function of time,

$$
H=\frac{H_{i}}{\left(1+4 \frac{\rho \Omega^{2}}{3 \eta} H_{i}^{2} t\right)^{\frac{1}{2}}},
$$

where $\mathrm{Hi}$ is the initial film thickness before spinning.

Significance of Analysis

The result obtained above shows that the final thickness of the spun film depends on several physical parameters, each of which will be discussed briefly. The final film height varies with the square root of the viscosity. Thus, a fourfold increase in solution viscosity will double the final film height. The solution viscosity is a strong function of concentration and is thus easily controlled. Also, The film thickness varies inversely with square root of solution density. As discussed previously, the density of liquid $\mathrm{CO}_{2}$ is dependent upon the operating temperature. In addition, the density also increases with increasing solute concentration. Finally, the thickness varies inversely with the rotational 
speed during coating. A large increase in rotational speed will dramatically lower the final film thickness.

The above analysis predicts the final wet film thickness. Calculation of the final dry film thickness is given by

$$
\mathrm{H}_{f}=\left(\phi_{o}\right) \mathrm{H}_{\text {wet }}
$$

where $\phi_{\mathrm{o}}$ is the volume fraction of polymer in the photoresist and $\mathrm{H}_{\text {wet }}$ is the wet film thickness [2]. Thus, if values for the solution viscosity, density, and solvent fraction are known, final film thickness can be predicted based only upon the initial film thickness, spinning time, and rotational speed.

\section{References}

1. Emslie, A.G., F.T. Bonnner, and L.G. Peck, Flow of a Viscous Liquid on a Rotating Disk. J. Appl. Phys., 1957. 29(5).

2. Bornside, D.E. and R.A. Brown, The Effects of Gas Phase Convection on Mass Transfer in Spin Coating. J. Appl. Phys., 1993. 73(2). 


\section{Appendix B. Design of the 8" $\mathrm{CO}_{2}$ Spin Coater}

Despite the impressive results of the first-generation spin coater, there were several obvious shortcomings of the apparatus that needed to be addressed. The concern of primary importance was the spatial limitations of the spin coating chamber, i.e., it could accommodate wafers no larger than 6" (125 mm) in diameter. 5" and 6" wafers are convenient for lab scale experiments, however, most current microchip fab lines utilize 8" wafers, with some next-generation fabs already switching to 12 " wafers. In order to further demonstrate the feasibility of this new $\mathrm{CO}_{2}$ based processing, and to smooth the transition from research to industrial application, it is necessary to spin 8 " wafers from $\mathrm{CO}_{2}$. To that end, a new spin coating vessel has been designed and constructed.

In addition to the larger vessel diameter, other improvements were made to the spin coating design. The spin coater used for the work in this thesis was very labor intensive to operate. Simply opening the chamber to add or remove a wafer required manually opening the device and lifting a $40 \mathrm{lb}$ stainless steel lid. While such an arrangement was effective, it made operation of the spin coater slower and physically demanding. To improve upon that, a new opening mechanism is used on the 8 " spin

coater, as shown in Figure B.1. The opening and lifting of the lid is accomplished using pneumatic controls.

Other improvements to the device include greater visibility in the form of larger and more numerous sapphire windows (Figure B.2) and a much smaller dead volume in the vessel. The reduction of vessel high, and thus total volume, will allow us to fill the 
chamber using much less $\mathrm{CO}_{2}$ (Figure B.3). This is not only economically sound, it also will speed up processing significantly, and allow a more rapid evacuation of the chamber after spin coating as well. In the previous setup, lengthy venting of $\mathrm{CO}_{2}$ would result in significant cooling due to the expansion of the $\mathrm{CO}_{2}$ gas. This cooling would occasionally freeze lines and valves, and could lead to significant condensation on the wafer itself. The smaller volume in the 8 " spin coater should reduce this problem.

The second-generation spin coater was of sufficient complexity that it could not be built locally. It was constructed by Uhde Hochdrucktechnik of Germany. Once this new vessel is installed, the spin coater should be a much more robust and powerful tool for research on spin coating in $\mathrm{CO}_{2}$. 


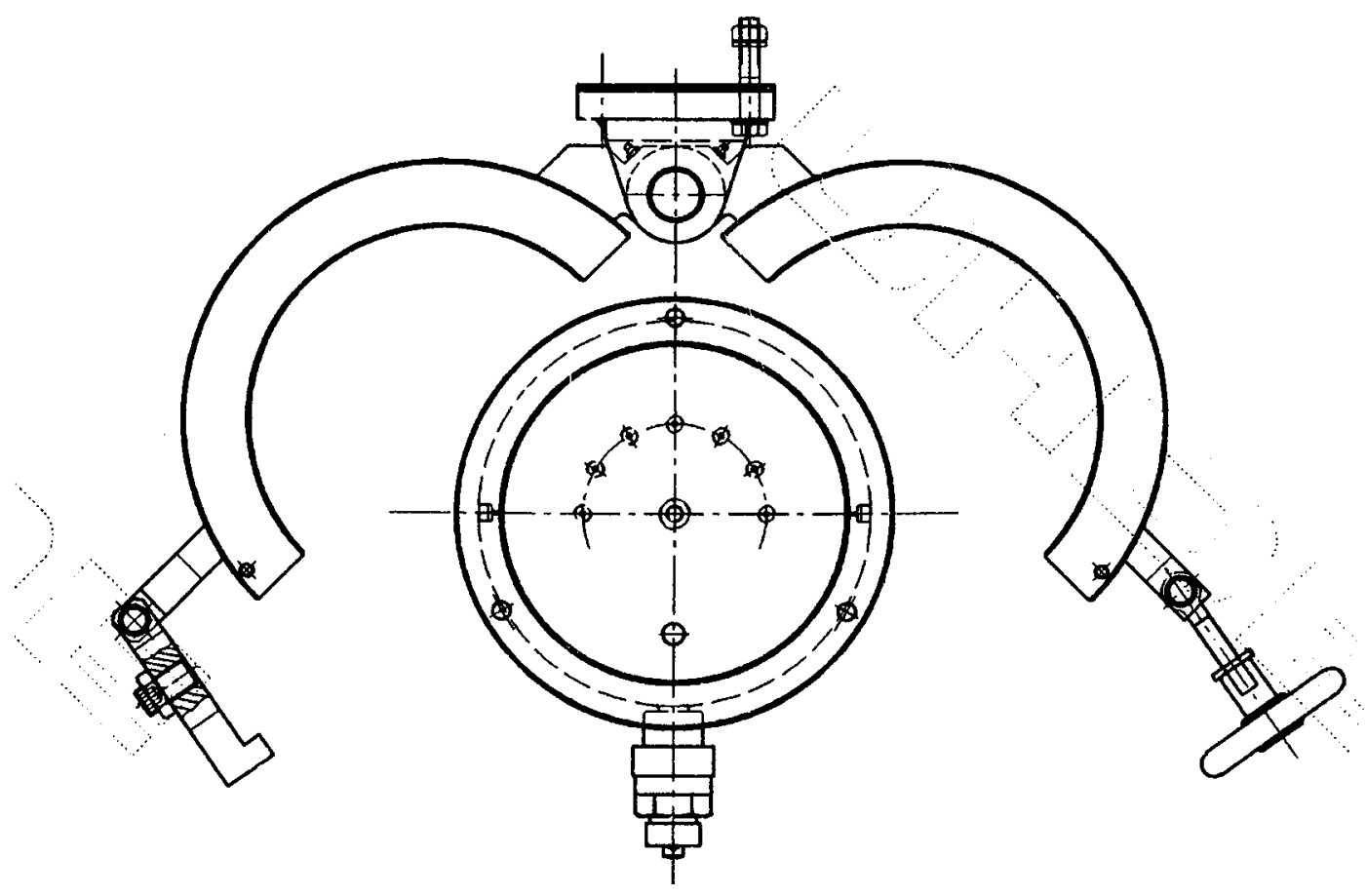

Figure B.1. Opening mechanism for the 8" spin coating vessel. 


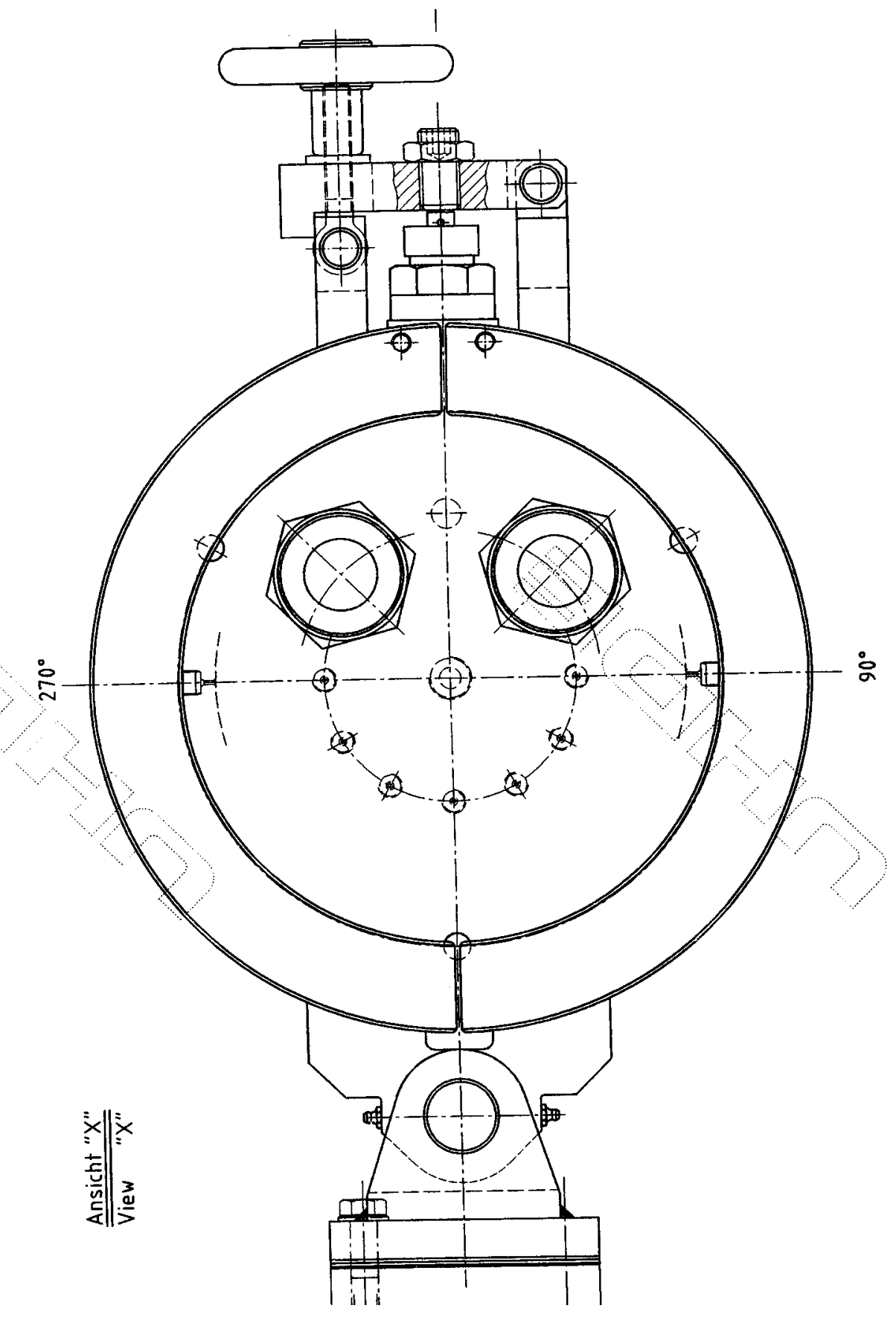

Figure B.2. Top view of the 8" spin coater. 


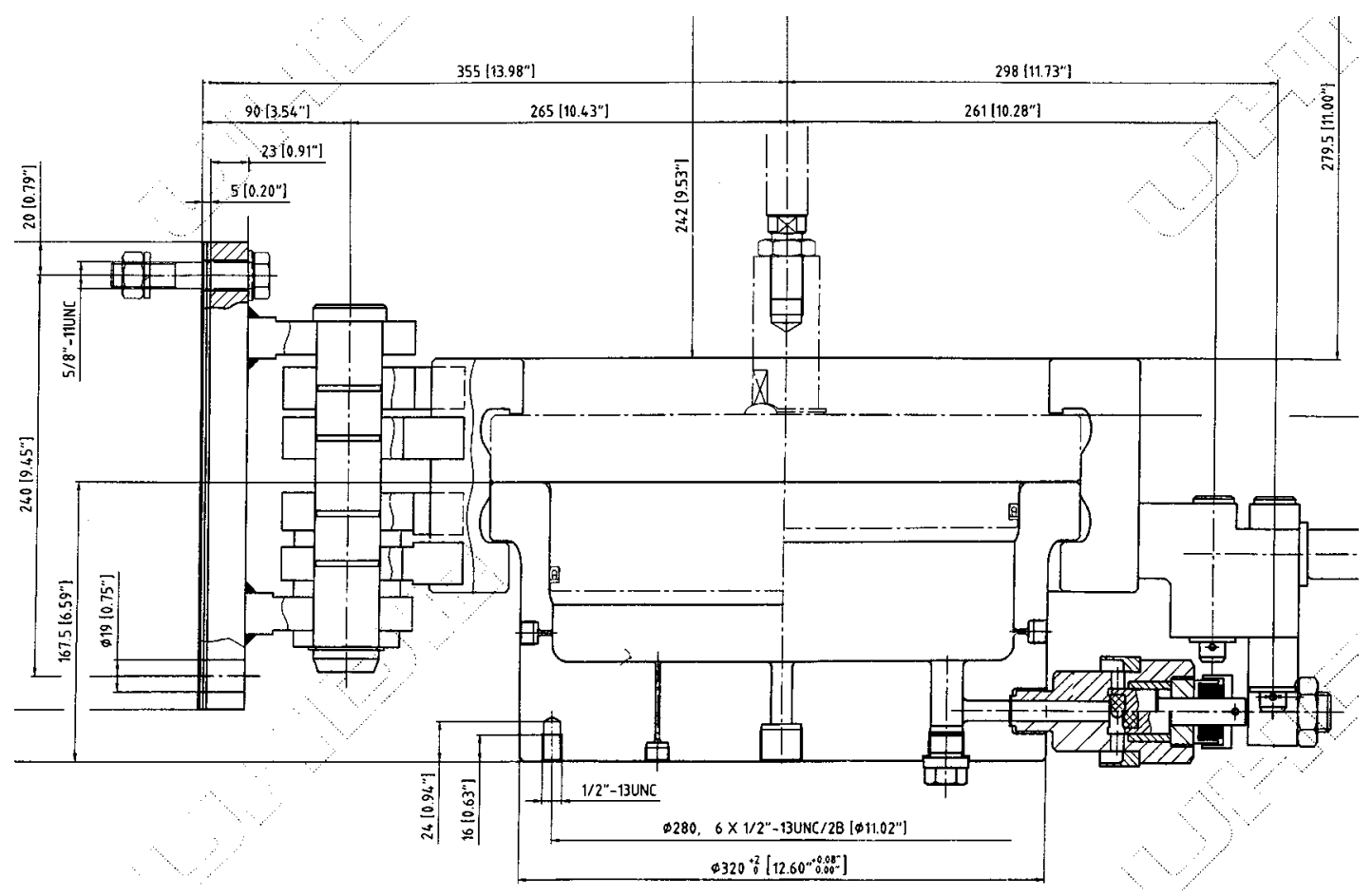

Figure B.3. Dimensions of the 8" spin coater - side view. 


\section{Appendix C. Metal Cleaning in Liquid $\mathrm{CO}_{2}$}

\section{Detergent-Aided Cleaning of Metal Surfaces in Liquid Carbon Dioxide}

Erik N. Hoggan, Ruben G. Carbonell, Gerald L. Cramer, Gina M. Stewart, Joseph M. DeSimone, published in Proceedings of CleanTech 98, 1998. 


\section{Abstract}

Carbon dioxide is a promising alternative to aqueous and halogenated solvents for cleaning operations. However, carbon dioxide cleaning has not been widely implemented because many contaminants are not soluble in pure liquid/supercritical $\mathrm{CO}_{2}$ except at high pressures and temperatures. The solubility of many contaminants in liquid/supercritical $\mathrm{CO}_{2}$ can be significantly enhanced by the addition of $\mathrm{CO}_{2}$-soluble detergents. This makes it possible to carry out cleaning operations in liquid $\mathrm{CO}_{2}$ at room temperature and pressures of approximately 900 psia. This paper describes the results of experiments comparing the effectiveness of metal cleaning operations using detergentaided liquid $\mathrm{CO}_{2}$ to cleaning done in aqueous and halogenated solvents under the same hydrodynamic conditions.

The rates of cleaning of aluminum coupons contaminated with films of cutting fluid (Mobilmet $\mathrm{Nu}$ [Mobil]) were measured. The cleaning experiments were conducted within a horizontal loading cleaning chamber with the coupons attached to a rotating element. The coupons were passed through the liquid/vapor interface at a range of rotational speeds. The same experiments were carried out in each of three media: an organic solvent (trichloroethylene), an aqueous detergent solution (2 vol\% Evercycle 2101 [Ransohoff] in $\mathrm{H}_{2} \mathrm{O}$ ) and liquid $\mathrm{CO}_{2}$ with 2 vol\% MICLEAN 102D [MICELL Technologies] detergent. The percent of the contaminant removed during cleaning was determined by non-volatile residue (NVR) analysis. 


\section{Introduction}

Manufacturing industries have traditionally used chloroflourocarbons (CFCs) and other halogenated solvents for the precision cleaning of metal surfaces [1]. The Environmental Protection Agency reported that in 1993 approximately 170 million pounds of CFC-113 and methyl chloroform were used in the United States alone for vapor degreasing operations [2]. As the use of these and many other traditional halogenated solvents is restricted due to increasing regulations over environmental concerns, alternative solvents such as perfluorocarbons and hydrochloroflourocarbons are being implemented in many cleaning processes. However, many of these replacement solvents are toxic, expensive, and may cause environmental problems. Because of these concerns, alternative cleaning technologies are being investigated.

Aqueous cleaning is replacing traditional solvents in most cleaning applications. Unfortunately, there are still several disadvantages to using aqueous cleaning. Performance rarely rivals that achieved when using halogenated solvents for the removal of oils. Large volumes of waste water may require treatment to minimize environmental impact. Residual water may be difficult to remove, making drying both time and energy intensive. In addition, the water sensitivity of many precision cleaning surfaces limits the applicability of aqueous cleaning. A promising alternative to both aqueous and solvent cleaning is the use of liquid or supercritical carbon dioxide in cleaning operations. 


\section{Physical Properties of Carbon Dioxide}

Carbon dioxide has several properties that make it an attractive solvent. Carbon dioxide is non-flammable, non-corrosive, non-toxic, inexpensive, renewable, and environmentally benign. The combination of a liquid-like density and a gas-like viscosity (Table G.1) make liquid/supercritical carbon dioxide an ideal choice for cleaning the complex surfaces present in many precision cleaning applications. The viscosity of liquid carbon dioxide is just $1 / 18^{\text {th }}$ that of water. This low viscosity results in relatively high transport rates. The liquid-like density of carbon dioxide $(70 \%$ that of water) maintains the high solute capacity of liquid solvents. Liquid carbon dioxide also exhibits a low surface tension. This allows the liquid to penetrate small crevices with greater ease than most solvents. In addition to these favorable properties, the use of carbon dioxide as a solvent eliminates many of the safety and environmental concerns present in cleaning processes.

Table C.1. Physical Data for Liquid Carbon Dioxide and Other Solvents [3]

\begin{tabular}{|c|c|c|c|}
\hline \multicolumn{1}{|c}{ Solvent } & Viscosity & Surface Tension & Density \\
& Centipoise & dynes/cm & $\mathrm{g} / \mathrm{ml}$ \\
\hline Carbon Dioxide, Liquid & 0.056 & 5 & 0.7 \\
\hline Trichloroethylene & 0.90 & 21 & 1.5 \\
\hline Methanol & 0.54 & 23 & 0.8 \\
\hline Water & 1.0 & 72 & 1 \\
\hline
\end{tabular}


The promise of liquid carbon dioxide as a solvent was recognized early in this century. However, cleaning in carbon dioxide has never been widely implemented because of several factors. The elevated pressures used to liquefy carbon dioxide require safety precautions. Also, cleaning in liquid/supercritical carbon dioxide requires specialized equipment, leading to higher capital costs. Perhaps most importantly, many contaminants are only slightly soluble in pure liquid/supercritical carbon dioxide. However, the use of novel detergents greatly increases the solubility of many of these contaminants in liquid carbon dioxide and makes carbon dioxide a much more attractive alternative solvent [4].

At ambient temperatures $\left(25^{\circ} \mathrm{C}\right)$ carbon dioxide is compressed to a liquid at 972 psia (Figure C.1). If the pressure is increased to 1070 psia and the temperature increased to $31^{\circ} \mathrm{C}$, carbon dioxide becomes a supercritical fluid. This low critical point makes it fairly inexpensive to drive carbon dioxide into the supercritical phase. However, all of the experiments discussed here are conducted at ambient temperatures, so the carbon dioxide is always in the liquid phase.

\section{Objectives}

These experiments were conducted as a comparison of three separate methods of cleaning aluminum contaminated with a cutting fluid. Trials were run using aqueous cleaning, cleaning with an organic solvent, and cleaning with detergent-aided liquid carbon dioxide. A control run was also performed using pure liquid carbon dioxide as the 
solvent. All of the experiments were conducted under the same hydrodynamic conditions in order to isolate the effect of solvent chemistry. By maintaining a constant shear rate in each of the three fluids, it is possible to compare directly the cleaning rates obtained in each solvent.

\section{Equipment}

The experiments were carried out in two different cylindrical cleaning chambers of identical dimension (3" ID x 12" length). The vessels were positioned horizontally and half-filled with solvent so that the rotating coupons passed through the liquid/vapor interface. Coupons were placed in a coupon holder that contained slots for up to 16 coupons, with the coated surface facing away from the shaft (see Figure C.2). One end of this coupon holder was connected to a drive shaft which extended through one end of the vessel. The aqueous and organic solvent experiments were conducted using a thin-walled stainless steel vessel sealed with Teflon ${ }^{\mathrm{TM}}$ o-rings. The liquid carbon dioxide cleaning trials required the vessel to be pressurized.

A cylinder of carbon dioxide ( $99.8 \%$ pure) was connected to a pump, which compressed the carbon dioxide to a liquid to fill the vessel. A high-pressure liquid level indicator (Jerguson) was used to monitor the amount of liquid carbon dioxide in the vessel. The coupon holder was attached to the shaft of an enclosed magnet drive unit [Magnadrive ${ }^{\circledR}$ II (Autoclave)], which could be operated over a range of rotational speeds. 
At the end of the cleaning cycle, the valve between the waste cylinder and vessel was opened, draining off much of the liquid. After the pressure dropped to around 200 psia, the waste cylinder was isolated, and the remaining carbon dioxide was vented directly to the atmosphere. A pressure gauge was used to monitor the pressure. A safety head with a rupture disc was attached to the vessel to vent off excess carbon dioxide in the event that the pressure exceeded the safety limit.

\section{Experimental Procedures}

All of the cleaning experiments were conducted using Aluminum coupons $1 \frac{1}{2}$ " $\mathrm{x}$ $1 / 2 " x 1 / 16 "$. The surfaces of the coupons were roughened by sandblasting for approximately 15 seconds to increase adhesion of the cutting fluid. The cutting fluid, Mobilmet $\mathrm{Nu}$, is a fairly low molecular weight hydrocarbon. Each coupon was coated with a thin layer of the cutting fluid $(\sim 140 \mathrm{mg})$. The fluid was dropped onto one face of the coupon and allowed to spread over the surface to a thickness of about $0.3 \mathrm{~mm}$. After coating, 8 coupons were placed on the coupon holder and inserted into the cleaning vessel. The vessel was then filled halfway with either the organic solvent [electronic grade trichloroethylene (Fisher)], the aqueous detergent solution [2 vol\% Evercycle 2101 (Ransohoff) in $\mathrm{H}_{2} \mathrm{O}$ ] or 2 vol\% MICLEAN 102D (MICELL Technologies) in liquid $\mathrm{CO}_{2}$.

The effectiveness of each cleaning method was determined using a non-volatile residue (NVR) analysis. After cleaning, the contaminant was dissolved using 5 vol \% isopropanol in trichloroethylene. The solution was then filtered using Magna nylon 0.45 
membrane filters (MSI) to remove impurities. After the solvent was evaporated, the nonvolatile residue was weighed using a Mettler AB204 analytical balance with an accuracy of $0.1 \mathrm{mg}$. Because coupons were analyzed in batches, data presented represent average values for 8 coupons.

The same coating and analysis procedures were used in all three media.

However, in order to isolate the effects of the solvent chemistry, it was necessary to modify the rotational speed used depending on the solvent properties. To ensure that the same hydrodynamic forces were acting on the contaminant in each case, the shear stress must be the same in each of the three media. The shear stress at the surface, $\tau_{\mathrm{o}}$, is the force per unit area applied in the direction parallel to the surface as a result of the movement of the coupon through the liquid phase.

To calculate the shear stress exerted by each of the fluids, the results of boundary layer theory for flow over a flat plate were used [5]. The boundary layer thickness, $\delta$, at a distance $\mathrm{x}$ over a semi-infinite plate is given by,

$$
\delta=\frac{5.0 \mathrm{x}}{\sqrt{\mathrm{Re}}} .
$$

Where $\operatorname{Re}$ is the Reynolds number $\left(\operatorname{Re}=\mathrm{xv}_{\infty} \rho / \mu\right)$. Here $\mathrm{v}_{\infty}$ is the fluid velocity relative to the plate, $\rho$ is the density of the liquid, and $\mu$ is the liquid viscosity (values given in Table C.1). The Reynolds number and the boundary layer thickness must be calculated to determine the conditions at the surface. For the coupons in the aqueous detergent rotating at $60 \mathrm{rpm}, \mathrm{Re}=1140$. This is well below the transition value of $2 \times 10^{5}$, so the flow should be laminar. Using this value we calculate the boundary layer thickness, $\delta$, to 
be $1.8 \mathrm{~mm}$. This is much larger than the coating thickness $(0.3 \mathrm{~mm})$, so all of the contaminant should be within the boundary layer. The shear stress, $\tau_{0}$, is given by [5],

$$
\tau_{\mathrm{o}}=\mu\left(\frac{\partial \mathrm{v}_{\mathrm{x}}}{\partial \mathrm{y}}\right)_{\mathrm{y}=0}=0.332 \mu \mathrm{v}_{\infty} \sqrt{\frac{\rho v_{\infty}}{\mu \mathrm{x}}} .
$$

Taking the average over the width of the plate, L, leads to an expression for the average shear stress over the entire surface of the coupon,

$$
\left\langle\tau_{\mathrm{o}}\right\rangle=0.664 \frac{\mu^{1 / 2} v_{\infty}^{3 / 2} \rho^{1 / 2}}{L^{1 / 2}} .
$$

By setting the shear stresses of the different solvents equal to each other, it is possible to calculate the ratio of rotational speeds required to generate equivalent forces on the contaminant,

$\left\langle\tau_{\mathrm{o}}\right\rangle^{\mathrm{I}}=\left\langle\tau_{\mathrm{o}}\right\rangle^{\mathrm{II}}$.

Where the superscripts denote fluid I and fluid II. Substituting the expression for $\tau_{\mathrm{o}}$ results in an equation relating the velocity ratio to the density and viscosity of each fluid,

$$
\left(\frac{v_{\infty}{ }^{I I}}{v_{\infty}{ }^{I}}\right)^{3 / 2}=\left(\frac{\mu^{I}}{\mu^{I I}}\right)^{1 / 2}\left(\frac{\rho^{I}}{\rho^{I I}}\right)^{1 / 2} .
$$

If fluid II is taken to be liquid carbon dioxide and fluid I is water, the ratio of velocities may be calculated using the values in Table C.1,

$$
\left(\frac{\mathrm{U}_{\infty}{ }^{\mathrm{CO}_{2}}}{\mathrm{U}_{\infty}{ }^{\mathrm{H} \mathrm{O}}}\right)=\left[\left(\frac{1}{0.056}\right)^{1 / 2}\left(\frac{1}{0.7}\right)^{1 / 2}\right]^{2 / 3}=2.94 .
$$


Therefore, the rotational speeds used for the carbon dioxide cleaning experiments must be nearly three times those used in the aqueous trials. If fluid II is taken to be trichloroethylene and the same calculation is repeated, the ratio of velocities for trichloroethylene and water is much closer to unity $(\sim 1.1)$.

Taking these calculations into account, the aqueous and trichloroethylene experiments were conducted at rotational speeds of 30 and $60 \mathrm{rpm}$. The carbon dioxide trials were done at 88 and $176 \mathrm{rpm}$. These ratios of rotational speeds ensure that there are no significant differences in the hydrodynamic forces acting on the coupons exposed to the different fluids.

\section{Discussion and Results}

Aqueous cleaning experiments were conducted with a commonly used detergent at a typical concentration ( 2 vol \% Evercycle 2101 in water). Several trials were conducted multiple times to determine the repeatability of the experiments. The results were repeatable within 3\%. This error of 3\% was consistent throughout all the experiments. Figure C.3 shows the weight percent of cutting fluid removed by aqueous cleaning. Also shown are the results of trials conducted at equal rpm using pure water. After spinning for 90 seconds at $60 \mathrm{rpm}$ in pure water, nearly $64 \mathrm{wt} \%$ of the oil was removed. As expected, the use of the detergent significantly decreased the amount of cutting fluid remaining on the surface; $86 \mathrm{wt} \%$ of the oil was removed under the same 
conditions when 2 vol\% Evercycle was used. However, even with the detergent, the cutting fluid was not entirely removed (99 wt \%) until 4 minutes of exposure.

Figure C.4 shows the results of experiments carried out in liquid carbon dioxide using the rotational speeds calculated previously. After cleaning the coupons under equivalent shear rates for 90 seconds in pure carbon dioxide, $77 \mathrm{wt} \%$ of the oil was removed. When the same procedure was repeated in liquid carbon dioxide with 2 vol \% MICLEAN 102D, $97 \mathrm{wt} \%$ of the oil was removed. Thus the carbon dioxide with detergent was even more effective than the aqueous solution.

Figure C.5 shows the results of cleaning experiments conducted using each of the three solvents. The trichloroethylene removed the contaminant completely after only 15 seconds at $30 \mathrm{rpm}$. As expected, trichloroethylene removed the contaminant in the shortest amount of time. The detergent-aided liquid carbon dioxide cleaned the coupons in slightly less time than the aqueous solution. After only 45 seconds the carbon dioxide removed $95 \mathrm{wt} \%$ of the contaminant. To achieve the same results with the aqueous detergent required longer times ( 2 minutes).

To improve the amount of contaminant removed using carbon dioxide, additional detergent may be used. MICLEAN 102D is soluble in carbon dioxide in fairly large amounts, but if too much detergent is used then proper rinsing becomes a concern. Therefore an intermediate value of $5 \mathrm{vol} \%$ MICLEAN 102D was used in the additional experiments. Results from a 5\% trial and a 2\% trial conducted under identical conditions are presented in Figure C.6. The change in detergent concentration accelerated the cleaning process. After only 45 seconds of cleaning in the $5 \%$ solution, $91 \%$ of the oil 
was removed. The same amount of cleaning in the $2 \%$ solution resulted in only $56 \%$ of the contaminant removed. Therefore, it is possible to improve the cleaning effectiveness of the liquid carbon dioxide by increasing the detergent concentration.

Additional cleaning trials were conducted using stainless steel coupons and an alternate cutting fluid, DO 4864C [ELF Atochem]. The same procedures were followed. Unfortunately, this highly viscous, chlorinated paraffin was not effectively removed by either the aqueous solution or the carbon dioxide. Even when rotated for several minutes, less than $5 \%$ of the cutting fluid was removed. However, this cutting fluid was cleaned by the trichloroethylene in a reasonable time period. Thus, although the detergent-aided carbon dioxide is an effective solvent for the Mobilmet $\mathrm{Nu}$, it appears to have difficulty solubilizing chlorinated contaminants with high viscosities. It would be necessary to use an alternate detergent to remove this and similar cutting fluids.

\section{Conclusions}

The results of these experiments are promising for the future use of liquid carbon dioxide as an alternative to traditional cleaning solvents. Under the same hydrodynamic conditions, the detergent-aided liquid carbon dioxide removed the Mobilmet $\mathrm{Nu}$ in equal or less time than the aqueous solution. As expected, detergent-aided liquid carbon dioxide did not clean as quickly as trichloroethylene, but with a slight increase in detergent concentration, carbon dioxide would be a suitable replacement for trichloroethylene in many cleaning applications. 


\section{Acknowledgements}

The authors would like to acknowledge the valuable assistance provided by Ramone Givens, of MICELL Technologies.

\section{References}

1. Weber, D., McGovern, W., and Moses J., "Precision Surface Cleaning with Supercritical Carbon Dioxide: Issues, Experience, Prospects," Metal Finishing, 93, pp. 22-26, March, 1995.

2. "Economic Impact Analysis of the Halogenated Solvent Cleaning NESHAP," U.S. EPA, 1993.

3. Howard, P. and Meylan, W. editors, Handbook of Physical Properties of Organic Chemicals, Lewis Publishers, Boca Raton, FL,1997.

4. McClain, J., Betts, D., Canelas, D., Samulski, E., DeSimone, J., Londono, J., Cochran, G., Wignall, G., Chillura-Martino, D., Triolo, R., "Design of Nonionic Surfactants for Supercritical Carbon Dioxide," Science, 274, pp. 2049-52, 20 December, 1996.

4. Geankoplis, C., Transport Processes and Unit Operations, $3^{\text {rd }}$ edition, Prentice Hall, Englewood Cliffs, NJ, pp. 192-193,1993. 


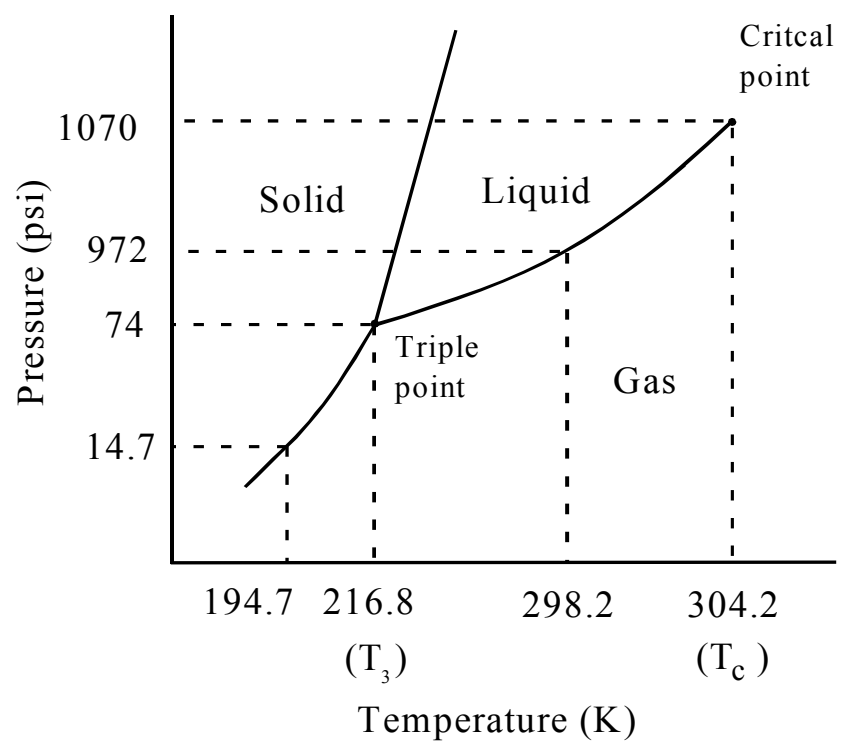

Figure C.1. Phase Diagram for Carbon Dioxide [3] (not to scale) 


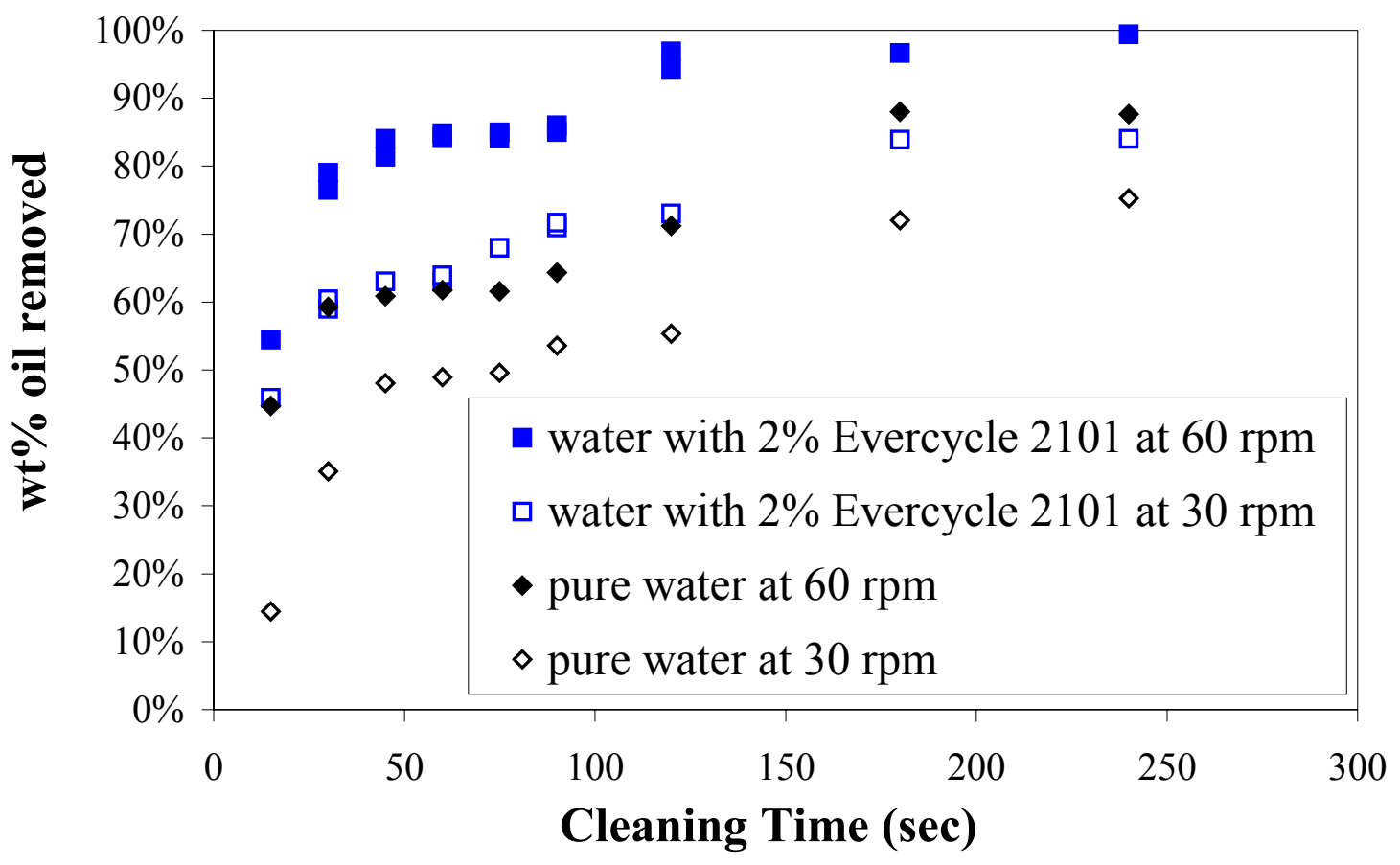

Figure C.2. Weight percent of oil removed as a function of time in aqueous system. 


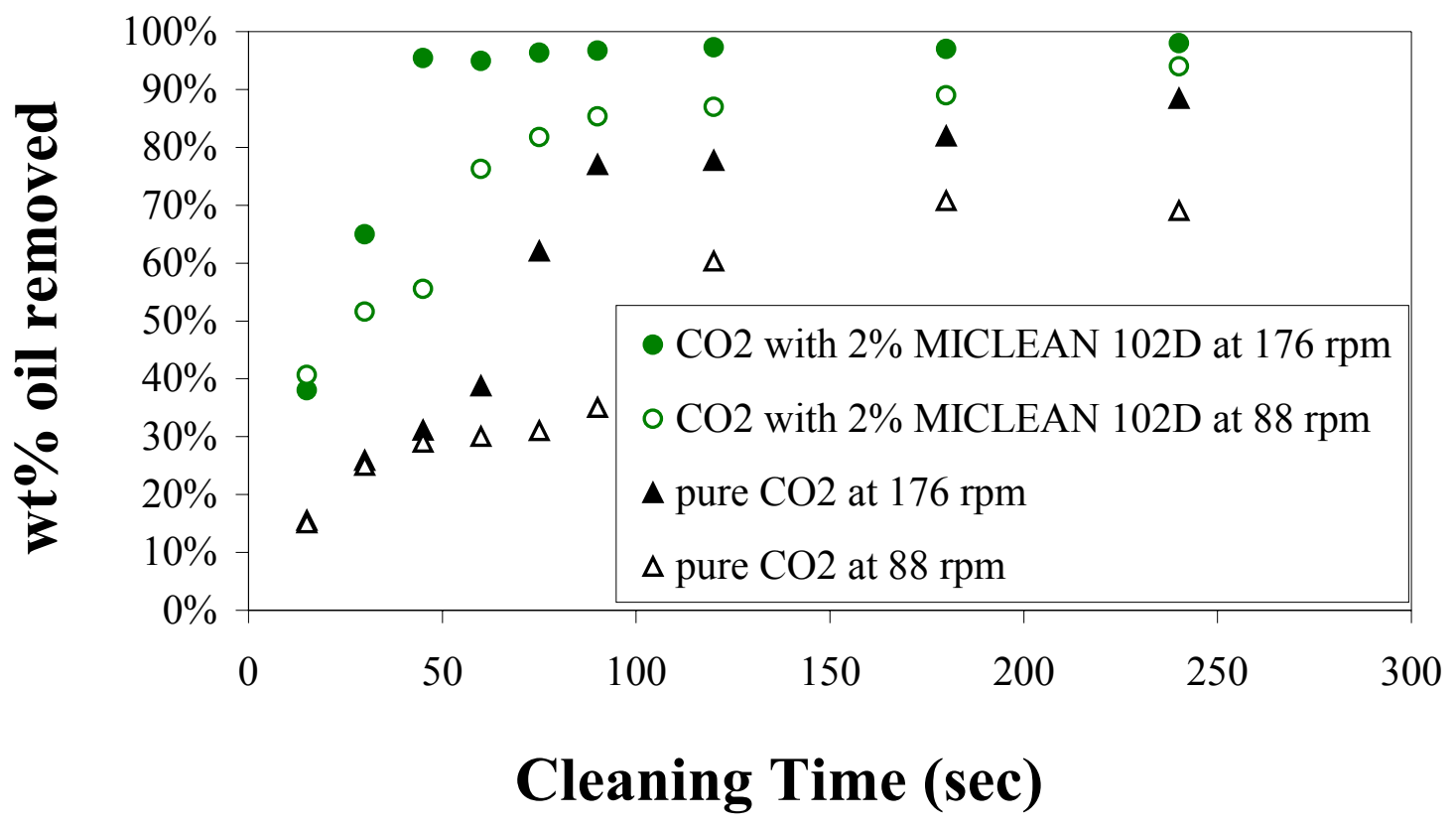

Figure C.3. Weight percent of oil removed as a function of time in carbon dioxide. 


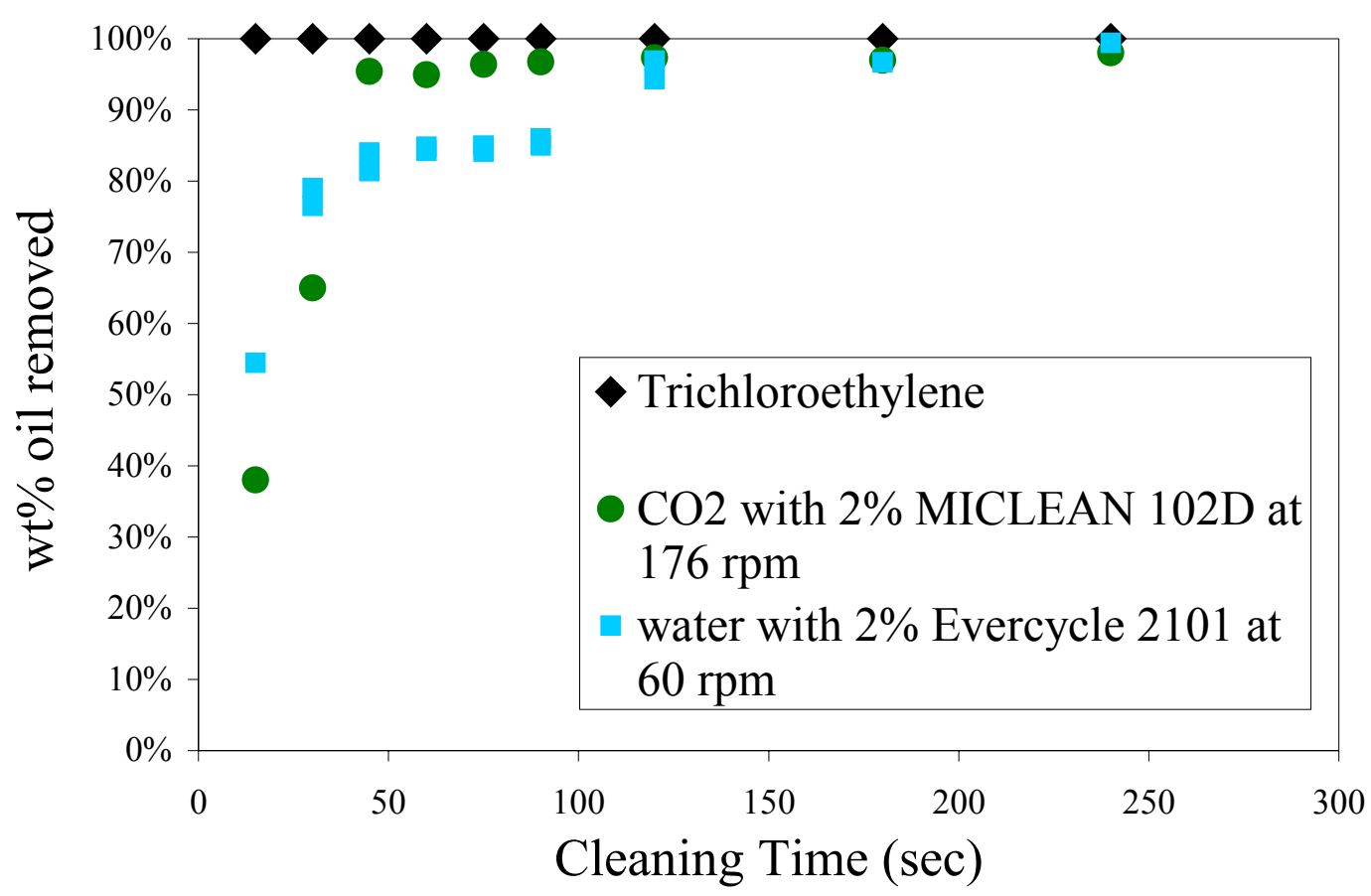

Figure C.4. Weight percent of oil removed as a function of time in three solvents. 


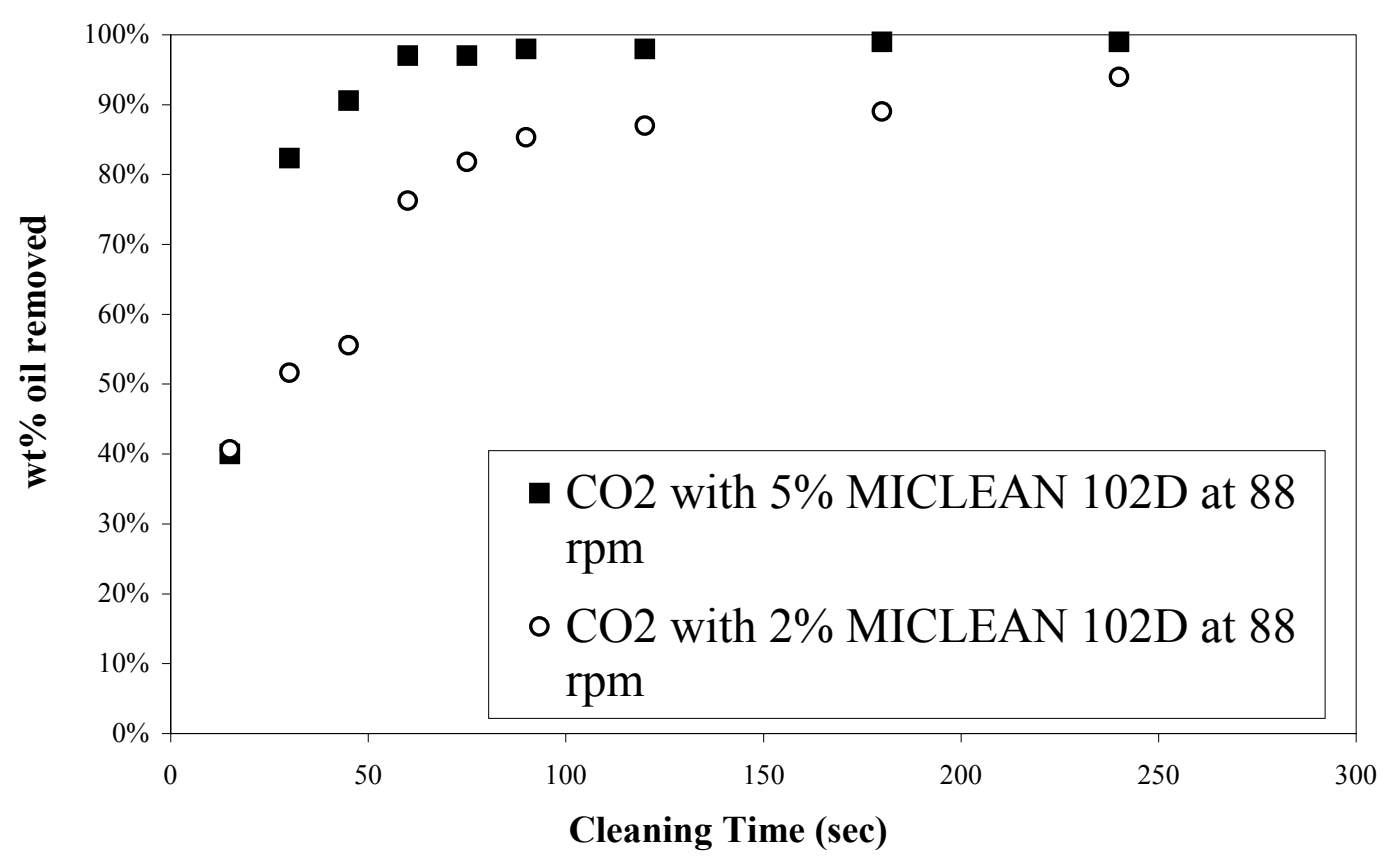

Figure C.5. Weight percent of oil removed as a function of time in carbon dioxide. 